\title{
Review Article \\ Fundamentals, Recent Advances, and Future Challenges in Bioimpedance Devices for Healthcare Applications
}

\author{
David Naranjo-Hernández $\mathbb{D}^{1},{ }^{1}$ Javier Reina-Tosina $\mathbb{D}^{1},{ }^{1}$ and Mart Min $\mathbb{D}^{2}$ \\ ${ }^{1}$ Biomedical Engineering Group, University of Seville, Seville, Spain \\ ${ }^{2}$ Tallinn University of Technology, Tallinn, Estonia \\ Correspondence should be addressed to David Naranjo-Hernández; dnaranjo@us.es
}

Received 12 October 2018; Revised 12 April 2019; Accepted 26 May 2019; Published 15 July 2019

Academic Editor: Andrea Cusano

Copyright (c) 2019 David Naranjo-Hernández et al. This is an open access article distributed under the Creative Commons Attribution License, which permits unrestricted use, distribution, and reproduction in any medium, provided the original work is properly cited.

\begin{abstract}
This work develops a thorough review of bioimpedance systems for healthcare applications. The basis and fundamentals of bioimpedance measurements are described covering issues ranging from the hardware diagrams to the configurations and designs of the electrodes and from the mathematical models that describe the frequency behavior of the bioimpedance to the sources of noise and artifacts. Bioimpedance applications such as body composition assessment, impedance cardiography (ICG), transthoracic impedance pneumography, electrical impedance tomography (EIT), and skin conductance are described and analyzed. A breakdown of recent advances and future challenges of bioimpedance is also performed, addressing topics such as transducers for biosensors and Lab-on-Chip technology, measurements in implantable systems, characterization of new parameters and substances, and novel bioimpedance applications.
\end{abstract}

\section{Introduction}

The continuous increase in life expectancy is contributing to an aging population and an increase in the prevalence of chronic diseases $[1,2]$. The consequence is an impact on the socioeconomic structure of society in terms of increased medical expenses and health and social welfare needs. This problem has driven new technological advances (sensing devices, low power electronics, wearable systems, communication technologies, etc.) to improve current healthcare systems and citizens quality of life [3]. Multiple sensorization technologies are being investigated and developed towards their application to the monitoring of the health condition, either in a clinical environment or in wearable devices for remote monitoring at the user's home $[4,5]$ : heart rate variability [6], glucose level in the blood [7], blood pressure [4], temperature [8], physical activity [2], pulse oximetry [9], etc. Among these technologies, bioimpedance takes a prominent place, since it allows providing an insight about the internal processes of the body in a noninvasive way $[10,11]$.

Bioimpedance techniques are based on the injection into a biological medium (human body, tissue, or cell culture) of an alternating electric current of very low intensity. The electric current produces a voltage drop, the higher, the greater the electrical impedance of the tissue [12]. Bioimpedance measurements are based on the fact that biological mediums behave as conductors, dielectrics, or insulators of electrical current, depending on their composition. The result of this phenomenon is a dependence of bioimpedance values with frequency, which can provide information on the physiology and pathology of tissues and cells.

One of the most outstanding applications of bioimpedance in healthcare is the estimation of body composition, which is normally applied to assess the nutritional and hydration status and useful in multiple clinical areas: obstetrics [13], postoperative monitoring [14], critical care [15], pregnancy and lactation [16], gastroenterology [17], nutrition [18], chronic inflammation [19], obesity [20], or sports science [21]. An area of great interest is nephrology since it has been shown that bioimpedance methods can help in the dialysis process to achieve the desired euvolemic state [12].

Impedance cardiography (ICG) is another bioimpedance application of great clinical interest related to the noninvasive monitoring of cardiac dynamics. The analysis of ICG signals 
is used in the diagnosis of patients with cardiovascular diseases [22, 23]. From ICG it is possible to obtain a series of parameters related to the mechanical function of the heart such as stroke volume, cardiac output, preejection period, left-ventricular ejection time, or systolic time ratio [22]. From bioimpedance measurements and more specifically by the analysis of pulse wave velocity (PWV), arterial stiffness can also be estimated, which is a parameter of interest in the diagnosis of hypertension situations or arrhythmia and stroke events [24]. Moreover, it can be used to find blood pressure indirectly [25].

The application of bioimpedance to obtain medical images of the inside of the human body should also be highlighted, which is known as electrical impedance tomography (EIT). In this method, harmless electric currents are applied on the subject skin and the resulting electric potentials are recorded with an electrode array [26, 27]. From these boundary voltage measurements, it is possible to reconstruct the internal volumetric conductivity distribution and images related to the impedance distribution inside a body, of great clinical interest despite its low resolution [28-30].

Bioimpedance methods are also the basis for clinical laboratory tools such as hematocrit meters, coulter counters, and cell culture monitoring systems, including Lab-onChip applications [31]. The bioimpedance spectrum reveals the electrical and mechanical properties of the cells such as growth, motility, activity, and viability [32, 33]. In cell cultures, bioimpedance has been used for the detection of interactions with drugs [34], the analysis of cell regulation [35], and its kinetics or the in vivo investigation of cancer models $[36,37]$.

Given their characteristics, bioimpedance measurements allow the characterization of biological media and organic tissues, including the human body, but also inorganic media [38]. In this sense, bioimpedance methods have been employed in skin monitoring (diagnosis of diseases and evaluation of a treatment progress) [38], cancerous tissue characterization and detection [31], sleep apnea detection through bioimpedance measurements [39], as a means of precisely controlling the energy delivered to the heart during defibrillation [40], as a predictor of intradialytic hypotension [41], for precise bone cement milling during revision of total hip replacement [42], for longitudinal knee joint health assessment $[43,44]$, for the evaluation of high-resolution temporal information corresponding to pharyngeal swallowing [45], to monitor the ischemia and viability of transplanted organs [46, 47], edema determination [48], brain and pulmonary function monitoring $[49,50]$, and even as a method for the noninvasive measurement of glucose level [51].

Bioimpedance devices are noninvasive, low-cost, portable, and user-friendly, practical advantages that have led to their rapid development. However, research in the field of bioimpedance is a very active area as more efficient and miniaturized instrumentation, novel models and algorithms, new parameters, and substance characterizations are required. It is important to optimize the sensors for sensitivity and power consumption, especially when used in Lab-on-Chip devices and implantable systems such as cardiac and other monitors and pacemakers. Repeatability and reproducibility are often limitations of this technology; thus, novel sensing techniques are necessary. Moreover, high frequency measurements require in-depth circuit design techniques to overcome challenges.

The purpose of this paper is to provide a review of bioimpedance fundamentals and applications and an updated record of state-of-the-art in bioimpedance technology for healthcare applications. The content of the review is organized in the following sections and subsections:

(i) Section 2: Measurement methods and devices

(a) Bioimpedance definition

(b) Bioelectrical basis of bioelectrical impedance

(c) Bioimpedance models

(d) Electrode designs and configurations

(e) Design and implementation of bioimpedance instrumentation

(f) Sources of artifacts and noise in bioimpedance measurements

(ii) Section 3: Bioimpedance for body composition assessment

(iii) Section 4: Impedance cardiography

(iv) Section 5: Transthoracic impedance pneumography

(v) Section 6: Electrical impedance tomography

(vi) Section 7: Skin conductance applications

(vii) Section 8: Impedance detection in biosensors

(viii) Section 9: Future challenges of bioimpedance

\section{Measurement Methods and Devices}

This section reviews the fundamentals of bioimpedance measurements, from the instrumentation and hardware used in the devices to the bioimpedance models that support the measurement algorithms. Given the importance of the electrodes in this type of systems, a section is included that covers the most outstanding aspects found in the bibliography related to this term. A final part reviews noise problems and artifacts that affect bioimpedance measurements.

2.1. Bioimpedance Definition. The impedance (Z), or bioimpedance when it comes to a biological medium, is defined as the ratio of a voltage over the electric current that it generates $(\mathrm{Z}=\mathrm{V} / \mathrm{I})$, and it represents the opposition of a conductor to the circulation of an electric current [12]. This property depends on the characteristics of the biological medium through which current flows, but also on frequency. Generically, bioimpedance is a complex number with electric resistance $(\mathrm{R})$ as real part and reactance $\left(\mathrm{X}_{\mathrm{C}}\right)$ as imaginary part, both expressed in $\Omega$.

$$
Z=R+j X_{C}
$$

$\mathrm{R}$ is described as the opposition of the medium to the flow of electric current and is inversely proportional to the 
conductance [52]. The other component, $\mathrm{X}_{\mathrm{C}}$, can be defined as the opposition of the medium to a change in electric current and is inversely proportional to the capacitance (biological mediums can be considered capacitive) [53].

\subsection{Bioelectrical Basis of Bioelectrical Impedance. Electricity} and chemistry are intimately related in biological media. As the electric current is transported by ions dissolved in the medium, it is not possible to explain the flow of electric current without taking into account the electrolytic theory [54]. Conductivity defines the amount of electricity that can flow and is directly proportional to the concentration of dissolved ions in the biological medium. The electric resistance $\mathrm{R}$ increases if the concentration of ions decreases $[52,55,56]$. The viscosity of the medium, an increase in the concentration of nonconductive substances or in the distance between the measuring electrodes, or a decrease in the effective area of the medium are also factors that contribute to the increase of $\mathrm{R}$. Other factors such as temperature or composition of the medium also influence the value of $\mathrm{R}$.

There is another property related to bioimpedance in a biological medium, the permittivity, which describes the ability of the medium to be polarized by an electric field [5557]. In dielectric media such as biological ones, a decrease in permittivity occurs as a consequence of the absorption of electromagnetic energy by the relaxation effects associated with the dipoles of the medium. At low frequencies, the changes produced in the electromagnetic field are slow enough to allow the dipoles to reach equilibrium before the field has reversed its polarity. From a certain frequency, the dipoles cannot follow the variations of the electric field due to the viscosity of the medium and a time delay is caused in the response of the medium, leading to the dissipation of energy in the form of heat. In biological media, three main dispersion regions can be distinguished in the frequency spectrum [55, 57-59]:

(i) $\alpha$ dispersion, normally in the frequency range between $10 \mathrm{~Hz}$ to $10 \mathrm{kHz}$. It is not fully understood yet but has been related to the phenomena of ionic diffusion of the cell membrane and the counterion effects $[59,60]$.

(ii) $\beta$ dispersion, in the frequency range between $10 \mathrm{kHz}$ and $100 \mathrm{MHz}$. It is mainly caused by the polarization phenomenon of cell membranes, whose behavior is similar to that of a capacitance $[52,58,59]$. The polarization phenomena of proteins and other organic macromolecules also contribute to this dispersion $[58,59]$.

(iii) $\gamma$ dispersion, in the range of gigahertz, caused mainly by the polarization of water molecules $[59,60]$.

The dielectric spectrum can then be modeled as the sum of five dispersions, giving rise to the following expression for the complex relative permittivity $\left(\varepsilon^{*}\right)$ depending on the angular frequency $(\omega=2 \cdot \pi \cdot$ frequency) $[61,62]$ :

$$
\varepsilon^{*}=\varepsilon_{\infty}+\sum_{n=1}^{5} \frac{\Delta \varepsilon_{n}}{1+j \omega \tau_{n}}+\frac{\sigma}{j \omega \varepsilon_{0}}
$$

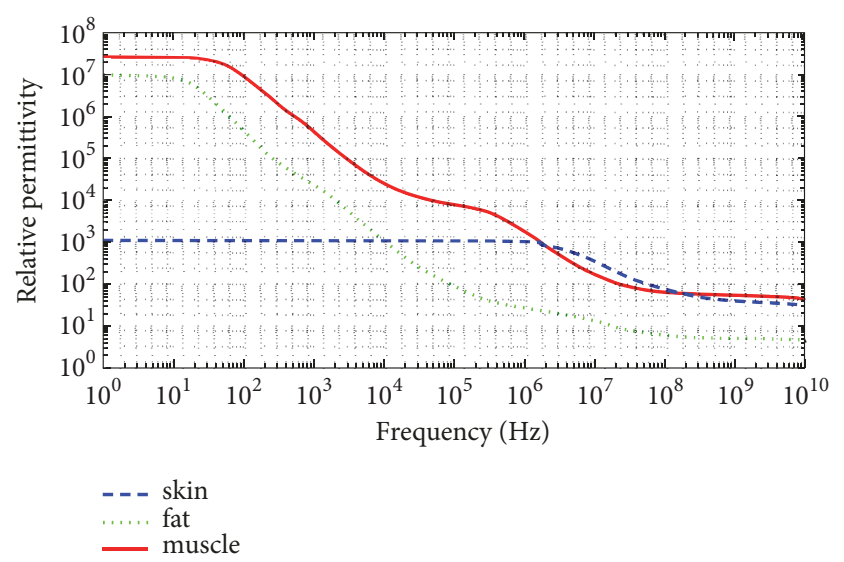

FIGURE 1: Relative permittivity of skin, fat and muscle [61].

where $\left(\varepsilon_{\infty}\right)$ is the permittivity at an infinite frequency, $\Delta \varepsilon_{\mathrm{n}}$ and $\tau_{\mathrm{n}}$ are the magnitudes and the time constants that characterize each dispersion ( $\mathrm{n}=1,2,3$ for $\alpha, \beta$ and $\gamma$ dispersions, respectively) [63], and $\sigma$ is the static ionic conductivity of the biological material. Figure 1 shows the dependence of the relative permittivity as a function of frequency for three body tissues (skin, fat, and muscle) according to the parameters defined in [61].

A more realistic model takes into account the complexity of biological materials, both in terms of composition and structure, through the inclusion of distribution parameters for of the time constants $\left(0<\alpha_{\mathrm{n}} \leq 1\right)$, according to the ColeCole model $[55,62]$ :

$$
\varepsilon^{*}=\varepsilon_{\infty}+\sum_{n=1}^{3} \frac{\Delta \varepsilon_{n}}{1+\left(j \omega \tau_{n}\right)^{\alpha_{n}}}+\frac{\sigma}{j \omega \varepsilon_{0}}
$$

The analysis of biological materials by bioimpedance is based on the study of the conductive or dielectric properties of the medium $[58,59,64]$. As the conductivity is directly proportional to the concentration of ions in the fluids of the medium, the electrolyte solutions of the body fluids (interstitial fluid, blood, lymph, etc.) and the soft tissues (muscles, organs, etc.) are good electrical conductors. The bone, the adipose tissue, and the air present in the lungs behave like a bad conductor (insulators). The frequency dependence in the bioimpedance differs from one tissue to another but also depends on the physiological state of the tissue (normal or pathological, alive or dead, dry or hydrated, etc.), so the impedance analysis is a suitable tool for the characterization of the state of the tissues [65].

2.3. Bioimpedance Models. Biological media are characterized by the presence of cells. In them, the intracellular medium, formed by intracellular water (ICW), and the extracellular medium, composed of extracellular water (ECW), can be distinguished [66]. Both media are conductive, as a consequence of the dissolved ions, and separated from each other by the cell membrane that acts as a dielectric [12]. This context affects the conduction of the electric current, and its behavior resembles that of a capacitance in which ECW and 


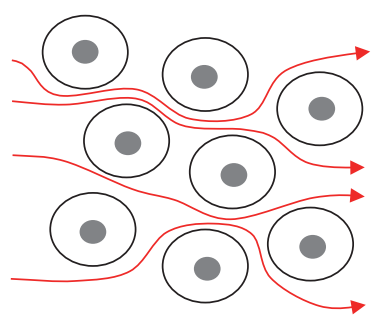

Low frequency

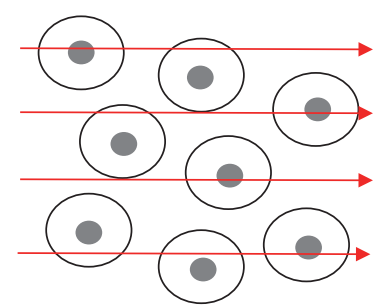

High frequency
Figure 2: Current flow in a biological medium at low and high frequency.

ICW are the plates of the capacitor and the membrane is the dielectric material [52]. For this reason, at low frequencies the current mainly flows through the space between the cells (see Figure 2). As frequency increases, a greater amount of current also penetrates in the intracellular medium, the more the greater the frequency.

Throughout the literature, a set of models have been proposed to respond to this behavior, which is directly related to bioimpedance.

(i) Single-dispersion $R C$ model: the simplest model of this behavior is a resistance $R_{E}$ that exemplifies the extracellular path of the current in parallel to a series disposition of a resistance $R_{I}$ and a capacitor $C$ to incorporate the frequency behavior of the intracellular path, with $\mathrm{C}$ representing the cell membrane and $R_{I}$ being the intracellular medium [66] (see (a) in Figure 3).

The bioimpedance in the s-domain can be expressed in terms of $\mathrm{R}_{0}$ (resistance at zero frequency) and $\mathrm{R}_{\infty}$ (resistance at infinite frequency) as follows:

$$
Z(s)=R_{\infty}+\frac{R_{0}-R_{\infty}}{1+\tau s}
$$

where

$$
\begin{aligned}
R_{0} & =R_{E} \\
R_{\infty} & =\frac{R_{I} \cdot R_{E}}{R_{I}+R_{E}}
\end{aligned}
$$

$s=j \omega, \omega$ is the angular frequency, corresponding to $2 \pi \mathrm{f}$, $\mathrm{f}$ is frequency in $\mathrm{Hz}$, and $\tau$ represents the time constant of the dispersion due to the capacitive component. For the single-dispersion RC model, the time constant $\tau$ is calculated by the following expression:

$$
\tau=\left(R_{I}+R_{E}\right) \cdot C
$$

If the bioimpedance values, from a zero frequency to an infinite frequency, are represented in the complex plane, a circular impedance curve is obtained (see Figure 4). The imaginary part of the bioimpedance is commonly represented with the opposite sign on the vertical axis, to avoid a representation in the fourth quadrant corresponding to a capacitive effect. The zero-frequency bioimpedance is purely resistive $\left(\mathrm{R}_{0}\right)$. As frequency increases, the position of the bioimpedance moves counterclockwise along the semicircle from $\mathrm{R}_{0}$ to $\mathrm{R}_{\infty}$.

(ii) Single-dispersion Cole model: according to fractional calculus [67], a general fractional component with impedance in the s-domain proportional to $s^{\alpha}$ can be defined. The traditional components of the circuits would be special cases of this component with a value of $\alpha$ equal to 1,0 and -1 for an inductor, resistor, and capacitor, respectively. A special case of the general fractional component is the constant phase element (CPE), whose impedance is $Z_{\mathrm{CPE}}=1 /\left(\mathrm{s}^{\alpha} \mathrm{C}\right)$ in the $s$ domain, where $\mathrm{C}$ is the value of the capacitance and $\alpha$ is its order. Its name refers to its phase angle $\varphi_{\mathrm{CPE}}$, which is constant at all frequencies and depends only on the $\alpha$ value $\left(\varphi_{\mathrm{CPE}}=\alpha \pi / 2\right)$. Typical values of $\alpha$ are in the range of $0 \leq \alpha \leq 1$ [68].

The Cole model is composed of three components (see (b) in Figure 3): a resistor at infinite frequency $\mathrm{R}_{\infty}$, a resistor $\mathrm{R}_{1}$, and a CPE. The bioimpedance is given by the following equation:

$$
Z(s)=R_{\infty}+\frac{R_{1}}{1+s^{\alpha_{1}} R_{1} C_{1}}
$$

The bioimpedance plot is also circular, but the circle center is located below the real axis of the impedance [12]. As it is shown in Figure 5, $\Theta=\alpha(\pi / 2)$ is the angle in which the center of the circle is displaced below the real axis. The cause of this effect comes from a distribution of relaxation times caused by the heterogeneity of cell sizes and shapes, the inaccuracy of the membrane behavior as an ideal capacitor, or physiological processes like the Warburg diffusion [54]. This model is very used because of its simplicity and good fit with bioimpedance measurements.

The single-dispersion Cole model and its parameters have been employed in various medical applications, since each parameter of the model has its own physical meaning [12, 69]: body composition analysis [70], measurement of the concentration of urea in the dialysate [71], tissue characterization [72-74] or analysis of blood samples [75, 76], ischemia monitoring $[72,77]$, hydration status evaluation [78], or cancer detection $[68,79]$. This model has also shown its utility in biology applications, fundamentally in plant physiology $[80,81]$ or the early detection of bacteria [69].

(iii) Extended single-dispersion Cole model: single-dispersion Cole model can be extended by introducing a time delay invariant with frequency $\left(T_{D}\right)$, which exemplifies the delays caused by the electronics and the measurement cables [82]. This effect is modeled by a 


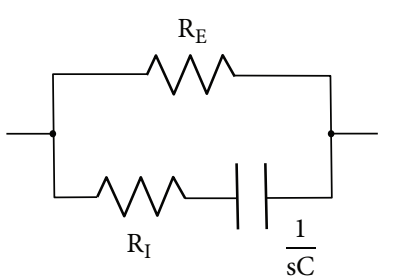

(a)

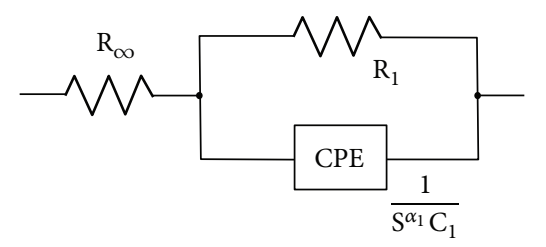

(b)

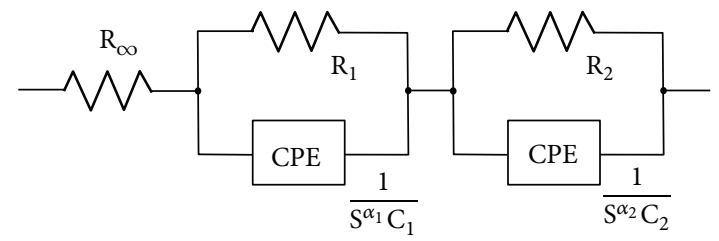

(c)

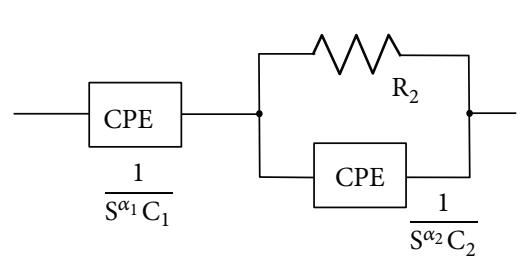

(d)

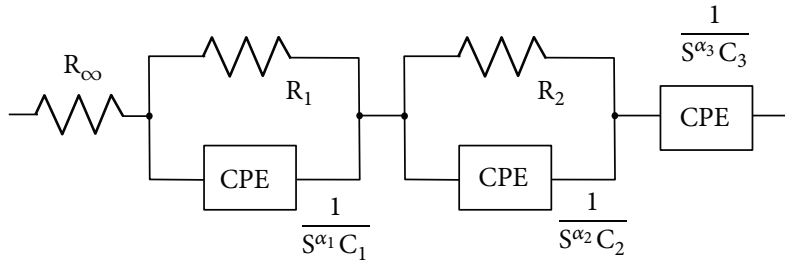

(e)

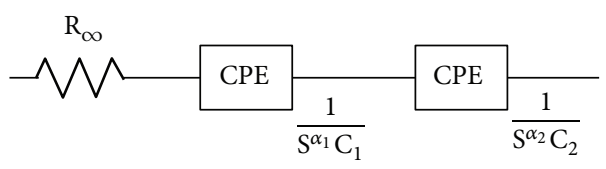

(f)

Figure 3: Bioimpedance models: (a) single-dispersion RC model, (b) single-dispersion Cole model, (c) double dispersion Cole model, (d) one resistor and two CPEs, (e) three resistors and three CPEs, and (f) one resistor and two CPEs, in series. $R_{E}$ and $R_{I}$ are the extracellular and intracellular resistances, respectively; $C$ is the membrane capacitance; $s$ is the complex variable in the Laplace domain; $R_{\infty}$ is the resistance at infinite frequency; CPE symbolizes a constant phase element; $R_{1}, R_{2}$ are the resistance increase due to the first and second dispersions, respectively; $\alpha_{1}, \alpha_{2}$, and $\alpha_{3}$ are the order of the fractional components associated with the first, second, and third dispersions, respectively; and $\mathrm{C}_{1}, \mathrm{C}_{2}$, and $\mathrm{C}_{3}$ are capacitances related to the first, second, and third dispersions, respectively.

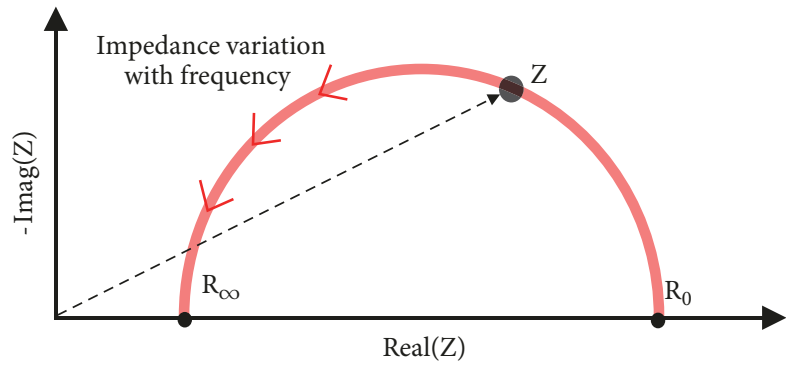

FIgURE 4: Typical bioimpedance plot.

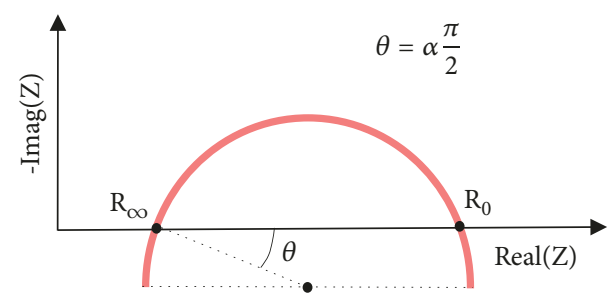

FIGURE 5: Bioimpedance plot of the single-dispersion Cole model.

linear phase increase with frequency as shown in the following expression:

$$
Z(s)=\left(R_{\infty}+\frac{R_{1}}{1+s^{\alpha_{1}} R_{1} C_{1}}\right) e^{s T_{D}}
$$

(iv) Double dispersion Cole model: this model allows representing the values of bioimpedance over a wider range of frequencies, where more than one dispersion can take place, or for more complex biomaterials [83]. This model is formed with a single-dispersion Cole model in series with a resistance and a CPE (see (c) in Figure 3 ) and the bioimpedance value responds to the following equation:

$$
Z(s)=R_{\infty}+\frac{R_{1}}{1+s^{\alpha_{1}} R_{1} C_{1}}+\frac{R_{2}}{1+s^{\alpha_{2}} R_{2} C_{2}}
$$

(v) Other fractional models: the model (d) in Figure 3 is formed by a resistance and two CPEs. This model has been used in the estimation of the internal moisture in wood [84], with a bioimpedance value given by

$$
Z(s)=\frac{1}{s^{\alpha_{1}} C_{1}}+\frac{R_{2}}{1+s^{\alpha_{2}} R_{2} C_{2}}
$$

The model (e) in Figure 3 has been used in the monitoring of root growth [85], which is formed by three CPEs and three resistors, with the following bioimpedance:

$$
Z(s)=R_{\infty}+\frac{R_{1}}{1+s^{\alpha_{1}} R_{1} C_{1}}+\frac{R_{2}}{1+s^{\alpha_{2}} R_{2} C_{2}}+\frac{1}{s^{\alpha_{3}} C_{3}}
$$

A resistor in series with two CPEs according to the model (f) in Figure 3 has been used in the modeling 


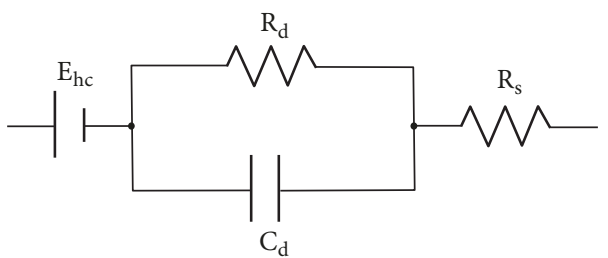

(a)

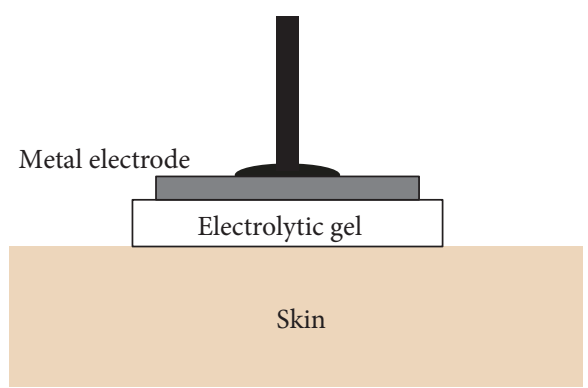

(b)

Figure 6: (a) Generic electrode model. (b) Electrode elements.

of the electrode-skin interface [86], with the following expression:

$$
Z(s)=R_{\infty}+\frac{1}{s^{\alpha} C_{1}}+\frac{1}{s^{\beta} C_{2}}
$$

(vi) Multiscale models: multiscale models have been proposed for modeling the dispersions of liver tissue. This model is built on a basic unit formed by resistors and capacitors [87], and through a hierarchical repetition of individual elements, from less to more, the different scales of the model are obtained: blood, liver cells, liver lobules, and liver tissue.

2.4. Electrode Designs and Configurations. A fundamental, but at the same time critical, aspect of a bioimpedance measurement system is the electrodes. They are the electrochemical interface necessary to put the biological medium in contact with the measuring equipment, both for the injection of current and for the detection of voltage. However, they are a major source of errors in bioimpedance measurements due to the stray capacitance and electrode polarization, the high contact impedance, the noise of the interface, and the artifacts due to movements, fluctuations, and contact problems [32, 88 ]. The impedance of the electrodes can be from hundreds of $\Omega$ to several $\mathrm{M} \Omega$, depending on frequency, but also on the characteristics of the electrodes [89]. One way to reduce the adverse effects is by maximizing the surface of the electrodes [32].

The design of a bioimpedance device starts from a good understanding of the behavior of the electrode-medium interface, thus the modeling of the electrodes has received a considerable interest $[88,90]$. Figure 6 shows a generalized electrode model with a single time constant [12]. In this model, $\mathrm{E}_{\mathrm{hc}}$ represents the double layer potential between the electrode and the electrolyte, when it exists, and between the electrolyte and the medium. $\mathrm{R}_{\mathrm{d}}$ is associated with the conduction currents due to the double layer and $C_{d}$ is associated with the displacement currents. $R_{s}$ represents the conduction losses in the electrolyte. More precise models employ two time constants, the first to model the skin, and the second for the behavior of the electrode itself [89].

For bioimpedance measurements on the human body, the electrode-skin contact usually consists of a metal electrode, an electrolytic gel, and the skin (see Figure 6). In these applications, the most common type of electrode is the Ag$\mathrm{AgCl}$ electrode, with an electrolyte based on $\mathrm{Cl}^{-}$[22]. Current injection and voltage measurement electrodes are usually located on the hand and on the foot of the same side of the body (global lateral position (a) in Figure 7), although other positions are possible, also with the electrodes on the hands and feet because of their better accessibility: (b), (c), (d), (e), and (f) in Figure 7 for bioimpedance measurements of one arm, trunk, left leg, right leg, or two arms, respectively.

For ICG measurements, Ag-AgCl electrodes are usually used in the Sramek configuration [91], in which two electrodes are located on the right lateral side of the neck, and two other electrodes on the left side of the thorax [22] (see (g) in Figure 7). In contrast, electrical impedance tomography typically uses an array of multiple electrodes uniformly distributed over the measurement area [26] (see (a) in Figure 8).

Apart from the $\mathrm{Ag}-\mathrm{AgCl}$ electrodes, other types of electrodes have also been used, such as the gold paste electrodes in the form of two concentric spirals presented in [38] to measure sweat on the skin. As an alternative to gel electrodes, capacitive electrodes have also been proposed as a measurement way that avoids direct contact with the user [92]. However, the results were not as accurate as in the traditional bioimpedance method because the impedance provided by the capacitive electrodes is much higher, in several orders of magnitude, than the impedance provided by the common direct contact electrodes and more susceptible to movement artifacts. Another example is the active electrodes based on a very low noise amplifier, with very high input impedance, and low output impedance, arranged directly on the electrodes [93].

Recently, textile electrodes have greatly increased their use in medical applications, including bioimpedance measurements, because they provide greater comfort to users, and to the extent that gel electrodes are not suitable for continuous use, they are disposable and can cause skin irritation [89, 94]. An example is the custom-made electrode garment described in [95], which is based on a biocompatible silverbased conductive fabric [95] for tetrapolar bioimpedance measurements between the wrist and the ankle.

In the case of microfluidic electrochemical sensors, coplanar electrode design is the most used configuration due to 


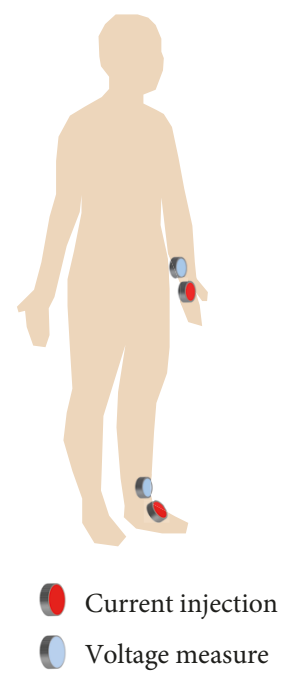

(a)

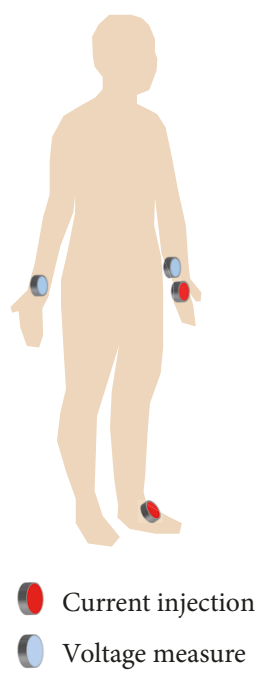

(b)

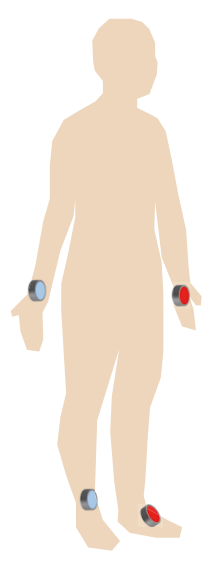

Current injection

Voltage measure

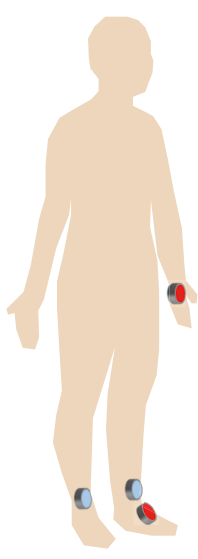

Current injection

Voltage measure

(d)

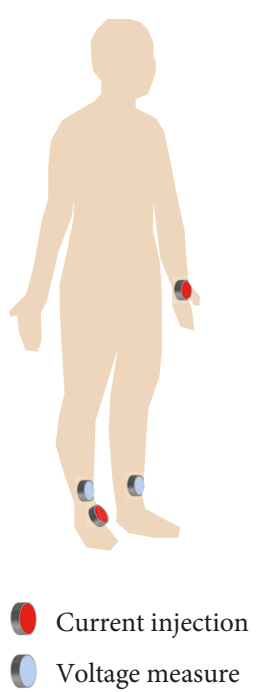

(e)

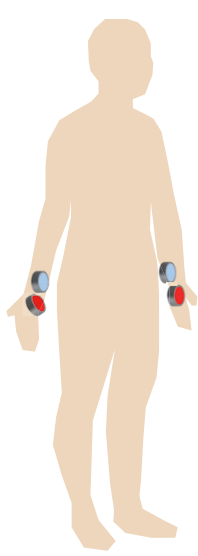

Current injection

Voltage measure

(f)

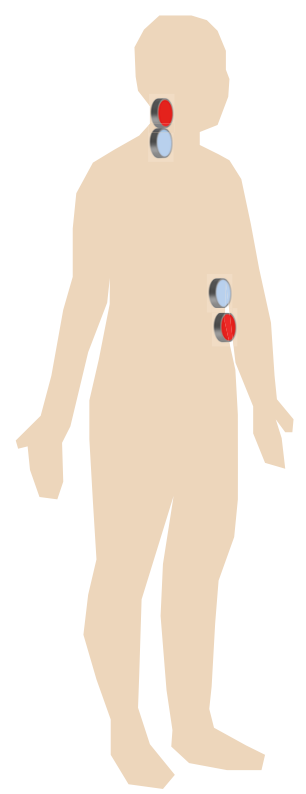

Current injection

Voltage measure

(g)

FIGURE 7: Typical electrode configurations for human body bioimpedance measurement: (a) global lateral, (b) one arm, (c) trunk, (d) left leg, (e) right leg, (f) two arms, and (g) example of electrode configuration for ICG measurement.

its easy manufacture. Nevertheless, the sensitivity of coplanar electrodes is limited as a result of the nonuniform field lines [32]. The incorporation of carbon nanofibers to the electrodes improves the sensitivity of these systems [96]. Another possibility is an arrangement of the electrodes in parallel plates [32].

For the characterization of biological media, the tetrapolar configuration is the most used because it minimizes the influence of the electrodes in the measurement process. This is the basis of the cylindrical chamber employed in [97] for the analysis of breast cancer cells, with circular electrodes at the ends as current electrodes and needle electrodes inside as voltage sensing electrodes [97]. In [68], the tetrapolar configuration was used on the same plane to study samples of breast tissue.

However, the tetrapolar electrode configuration can be affected by the anisotropy of the tissue such as nerve fibers and muscle or blood vessels. Measurement schemes that include multiple orientations have been proposed in the literature, as the case shown in [98], in which sixteen 


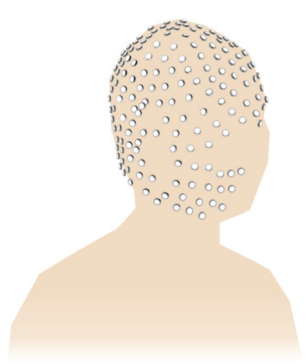

(a)

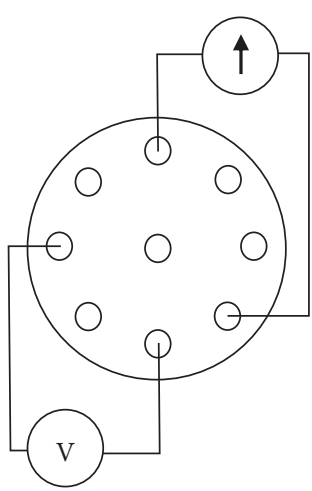

(b)

FIGURE 8: (a) Example of electrodes placement for electrical impedance tomography. Electrical impedance tomography of human head. (b) Example of electrode configuration for the simultaneous measurement in different orientations [37].

miniature electrodes perform sequential tetrapolar measurements to recover anisotropic conductivity tensors [98]. Another example is shown in [37], in which a nine-electrode microendoscopic probe was used (see (b) in Figure 8). In both cases, current injection and voltage measurement were carried out sequentially in different electrodes following a preconfigured pattern [37]. In [98, 99], new methods for measuring the anisotropy of tissues using bioimpedance have been proposed by modeling the anisotropy in both the resistivity and reactivity in three or more different directions. The anisotropy measurement has the potential to provide new insights in medical evaluation and diagnosis. In [100] a 16-electrode probe was used to reconstruct the anisotropic impedance spectrum allowing discriminating neurogenic and myopathic diseases. In other cases, however, a spiral structure has been employed in the electrodes to eliminate the anisotropic properties of the skin and measure the impedance independently of the direction in which the sensing device is located [38].

The polarity of the electrodes has also been analyzed in an application that uses bioimpedance as a guidance method during the craniotomy [101]. According to these results, a bipolar scheme, in which two opposite currents are injected into the medium at the same time in two different positions, is more sensitive than a monopolar scheme, with a single injection point.

2.5. Design and Implementation of Bioimpedance Instrumentation. The basis of a bioimpedance device is the injection of a current into a biological medium, at one or more frequencies, and the measurement of the voltage produced by the circulation of that current [102]. If the biological medium is the human body, in order to avoid any possible harmful effect, the international safety standards specified in standard IEC 60601 must be strictly complied [31, 95, 103]. For this reason, most devices inject a current less than $1 \mathrm{~mA}$ $[45,103]$.

The standard measurement frequency of bioimpedance devices is $50 \mathrm{kHz}[31,104]$. However, due to the interest of the frequency behavior of bioimpedance [31,33], some devices analyze multiple frequencies by means of a more complex instrumentation. The frequency of the injected current can be generated by an oscillator [43, 105], but it can also be generated digitally by means of a digitalto-analog converter (DAC) [106, 107]. A voltage-controlled current source (VCCS) transforms this oscillating signal into a suitable current for its injection into the biological medium $[43,105,106]$.

The injected current can be monitored to avoid exceeding the established limits [44], but the most common method is the injection of a fixed amplitude current [108]. Although multiple current source topologies have been employed for the VCSS, most are based on the improved Howland current pump $[102,106]$, implemented by discrete designs based on operational amplifiers (OPAM). However, this topology is not practical for integrated circuits (IC) due to the need for high-value precision resistors [109]. In this case, designs based on transconductors [110] and integrated current drivers fabricated in complementary metal-oxidesemiconductor (CMOS) technology have been proposed [111]. The desirable characteristics for the design of the VCCS are a constant current for variable loads and a high output impedance, especially in the case of EIT [112].

Bioimpedance measurements are mainly performed in two different configurations, depending on the number of electrodes [33, 45, 68, 107, 113]: two and four electrodes. When only two electrodes are used, the voltage is measured at the same current injection electrodes. This configuration is typical in the monitoring of cell cultures by electrochemical impedance spectroscopy (EIS), where the presence of a particle perturbs the electric field lines, and therefore the impedance, between two coplanar electrodes [96].

As the impedance of the electrode-medium interface is in series with the bioimpedance to be measured, important errors may arise. To solve this problem, the four-electrode configuration (tetrapolar) is usually employed [107, 108]. In this scheme, the current is injected by the external electrodes (distal electrodes) and the voltage is measured in the internal electrodes (proximal electrodes) (see Figure 9) by means of an instrumentation amplifier. Since the input impedance of 


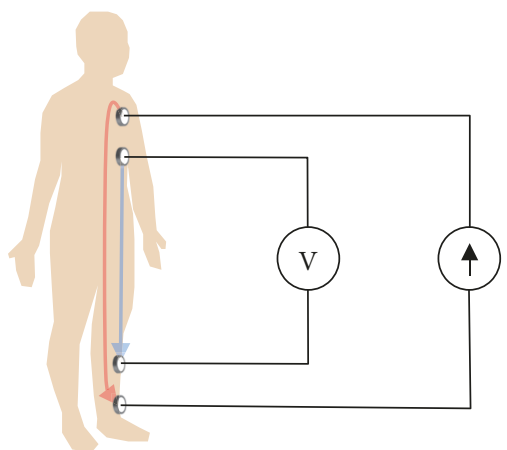

FIGURE 9: Four electrodes configuration.

the instrumentation amplifier is very high, the voltage drop at the sensing electrode can be considered negligible [36].

Bioimpedance can be evaluated in multiple ways by the measurement instrumentation. Below, some of the most relevant bioimpedance measurement schemes are presented:

(i) Modulus measurement: when only the temporal variation of the impedance modulus is of interest, it is common to perform an amplitude demodulation (bioimpedance variations can be seen as the modulation of a carrier signal in this case). The magnitude can be evaluated by peak detection [31, 114] or by rectification (half-wave or full-wave) with subsequent low-pass filtering [45].

(ii) Phase measurement: if a square current signal is generated, the comparison of the original signal and the measured signal can be used to detect the phase of bioimpedance [115]. XOR or SR latch schemes have also been employed to generate clock signals with pulse width dependent on the phase shift [116].

(iii) Quadrature demodulation: this method is based on two multiplications of the measured voltage signal, one by an in-phase signal $\left(0^{\circ}\right)$ and another by a quadrature signal $\left(90^{\circ}\right)[43,44]$. The output of each multiplier is filtered to obtain the DC components, and from them, the modulus and the phase of the impedance are estimated. The scheme of a bioimpedance meter based on quadrature demodulation is shown in Figure 10. The order and the cutoff frequency of the filter are established by the bioimpedance signal to be monitored [117]. If the bioimpedance changes slowly, a single multiplication can be used by alternating the phase between $0^{\circ}$ and $90^{\circ}[31]$.

(iv) Intermediate frequency approach: the high bandwidth required by the signal sensing channel has a negative effect on power consumption. To reduce this effect, the bioimpedance frequency can be transferred to a lower intermediate frequency before amplification. This procedure is generally carried out in two phases: first, the signal is transferred to baseband, and next the signal is modulated at an intermediate frequency. In [31] an alternative method composed of a single phase was proposed. Finally, the signal is demodulated in amplitude or quadrature to obtain bioimpedance information.

(v) Synchronous sampling: since the amplitude, phase and frequency of the signal are known parameters, it is possible to obtain the complex bioimpedance value by sampling the signal at the instants in which the acquired signal reaches the peak values and zerocrossings [118]. This procedure requires a very precise synchronization and makes the measurement rather challenging at high frequencies [31].

(vi) Oscillation-based method: this method converts the medium under test into an oscillator. Any variation in the bioimpedance produces a variation in the oscillation frequency, which is easily measurable with minor hardware resources $[33,119]$. In addition, the need to generate a stimulation signal is avoided. To maintain the oscillation condition, a nonlinear element (a simple comparator) is included in the feedback loop. LC oscillators have also been used in the monitoring of bioimpedance changes by displacements of the resonance frequency [119].

(vii) Impedance bridge method: in this method, an impedance bridge topology is used to estimate the bioimpedance of the biological medium, represented in Figure 11 as $Z_{B}[12,120]$. Its value can be estimated by the following expression:

$$
Z_{B}=\frac{Z_{1} Z_{3}}{Z_{2}}
$$

where $Z_{2}$ and $Z_{3}$ have known values and $Z_{1}$ is tuned until no current flows through $M$.

Current implementations of this method employ a simplified scheme known as self-balancing method [121]. A typical implementation is shown in Figure 12, where two voltage sources are used $\left(V_{1}\right.$ and $\left.V_{2}\right), Z_{1}$ is the impedance to calculate, and $R_{2}$ is a resistance of known value [121]. The signals $\mathrm{V}_{1}$ and $\mathrm{V}_{2}$ respond to the following expressions:

$$
\begin{aligned}
& V_{1}=A_{1} \cdot \sin (2 \pi f t) \\
& V_{2}=A_{2} \cdot \sin \left(2 \pi f t+\varphi_{2}\right)
\end{aligned}
$$

The value of $A_{1}$ is known and fixed and the values of $A_{2}$ and $\varphi_{2}$ are modified until a balanced voltage is obtained in the $\mathrm{G}$ terminal. Then, the bioimpedance value can be calculated with the following equation:

$$
Z=R_{2} \frac{A_{1}}{A_{2}} \angle \varphi_{2}
$$

Variants of the self-balancing method have employed an instrumentation amplifier and a DAC to get the balance by feedback [122] or a scheme based on an adjustable digital potentiometer as reference and instrumentation amplifiers for measuring voltage values in a four-electrode configuration [123]. 


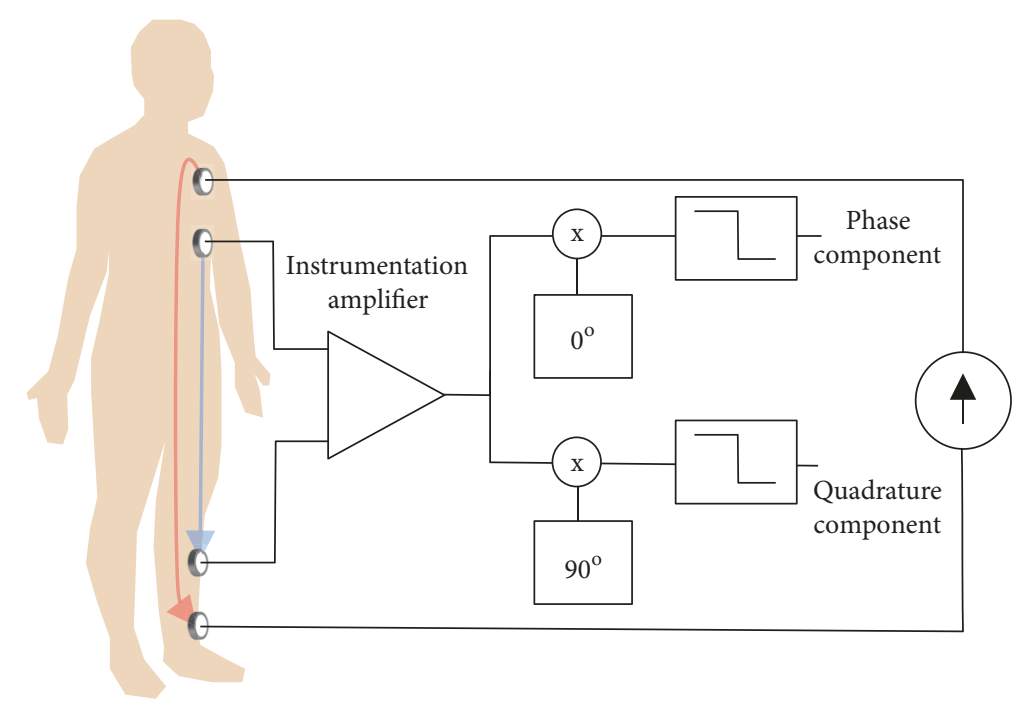

FIGURE 10: Quadrature demodulation method.

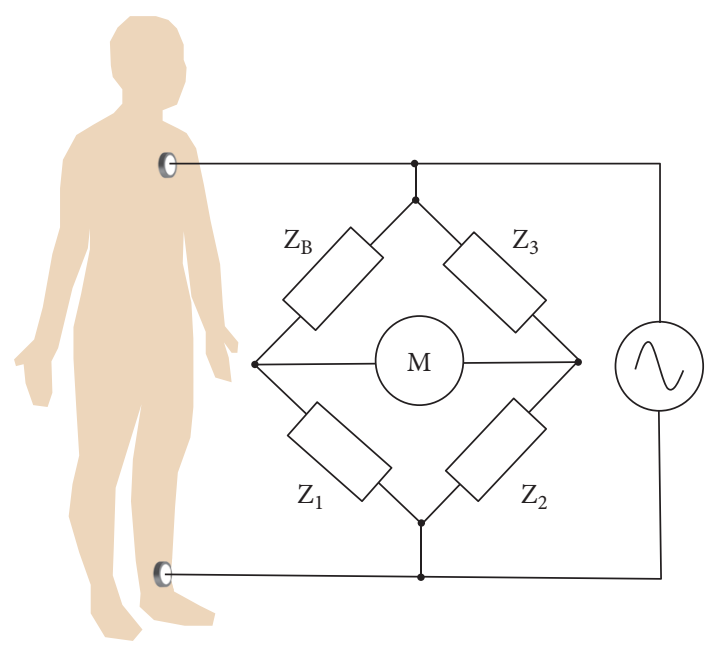

FIGURE 11: Impedance bridge method.

(viii) Amplitude-to-time conversion: this method has been used in the evaluation of the gain/attenuation with respect to the reference voltage applied in EIT measurement systems [124]. It is based on the fact that sinusoidal signals of different amplitudes intercept each other with a fixed delay with respect to the reference signal, as shown by (18).

$$
A \cdot \sin (2 \pi f t)=\sin (2 \pi f t+\varphi)
$$

Figure 13 shows the scheme proposed in [124], in which a reference sinusoidal signal is generated as an offset copy of the excitation signal. Comparator A generates a square signal whose active value represents the condition that occurs when the delayed reference signal takes a negative value (see Figure 14). The square signal resulting from comparator B reflects

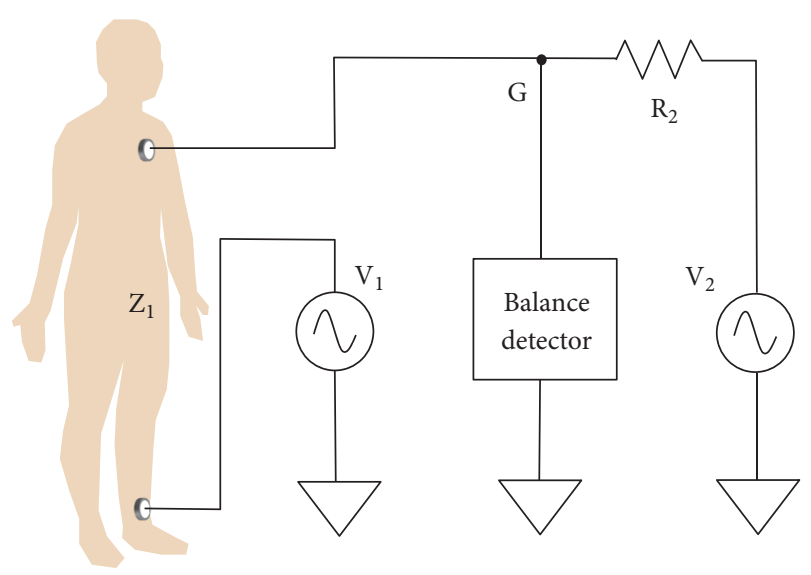

FIGURE 12: Self-balancing method bridge method according to [121].

the intersections of the reference signal with the measured signal. The time duration of the period in which the signals from comparator A and comparator $B$ are active at the same time is related to the gain of the measured signal. The relationship between this time measurement and the gain also depends on the phase shift applied by the phase-delay unit, and a calibration can be performed to adjust this parameter [124].

(ix) Fast Fourier Transform (FFT): FFT has also been used to obtain the bioimpedance spectrum using a multisine excitation [125], chirp signals [126, 127], or white noise [128].

(x) Orthogonal sequential digital demodulation method: in this method, two or more frequencies are employed, and subsequently, a digital demodulation based on the orthogonal property of the triangle function is performed $[129,130]$. 


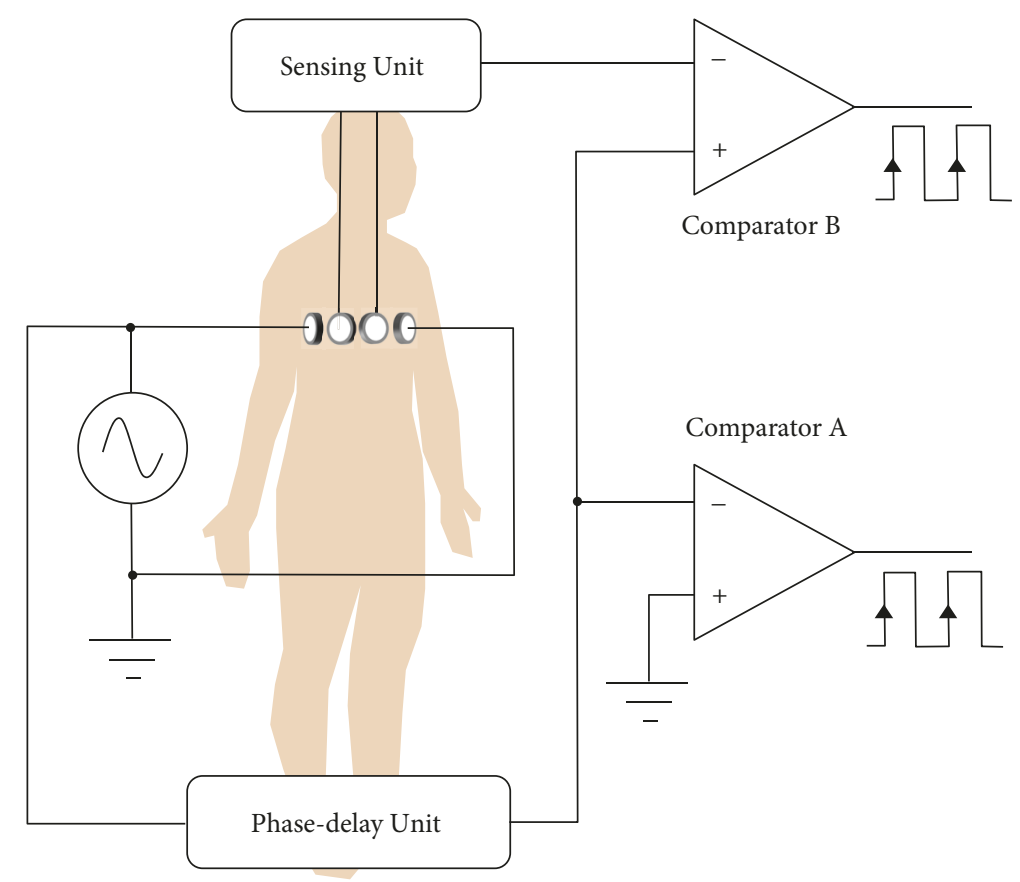

Figure 13: Amplitude-to-time conversion method proposed in [124].
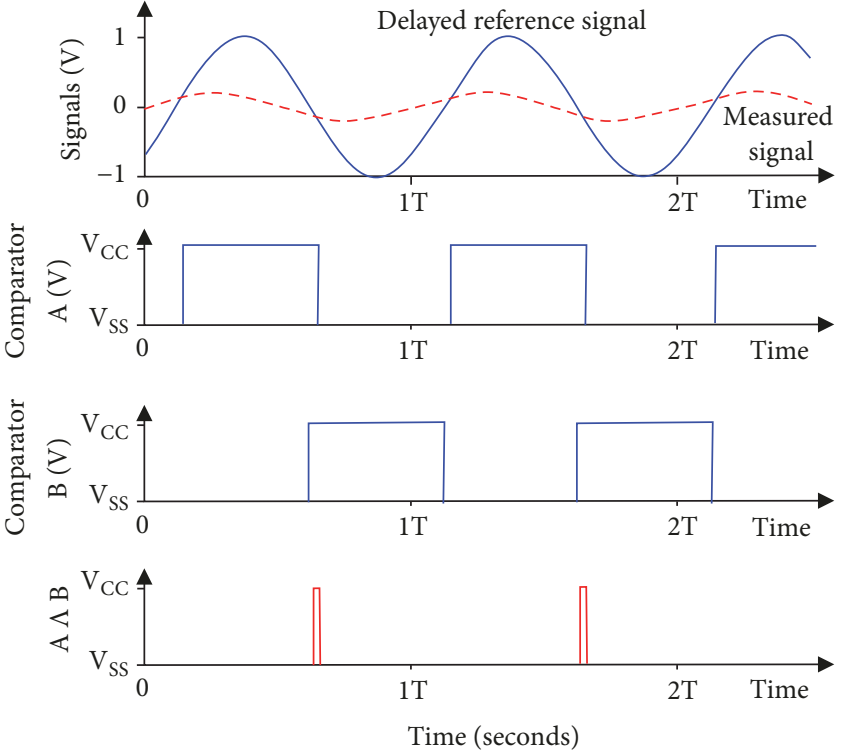

$\mathrm{V}_{\mathrm{CC}}$ : Positive supply voltage

$\mathrm{V}_{\text {SS }}$ : Negative supply voltage

(a) Gain $=0.2$
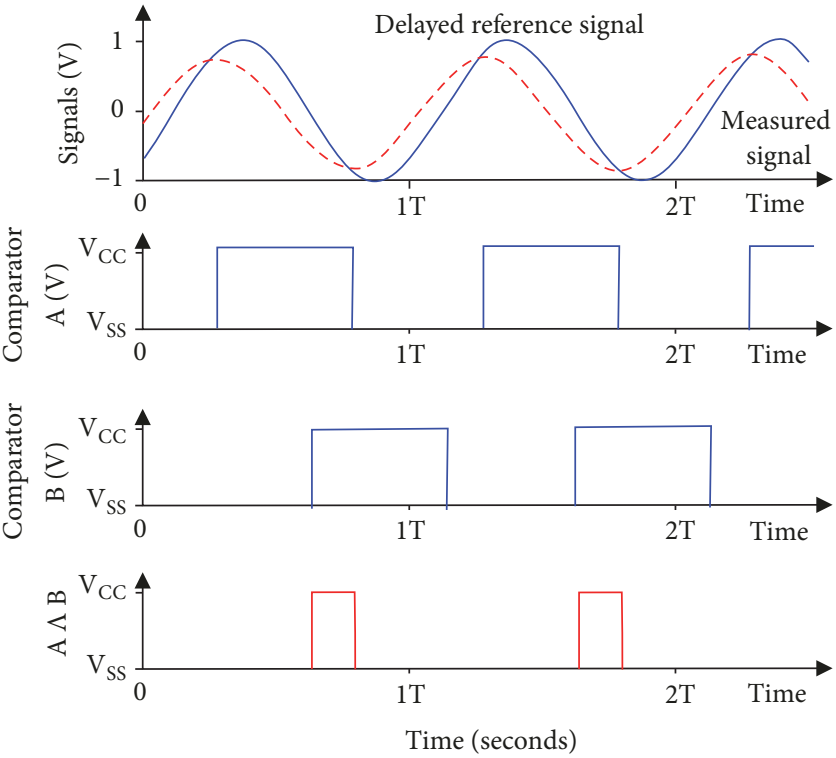

$\mathrm{V}_{\mathrm{CC}}$ : Positive supply voltage

$\mathrm{V}_{\mathrm{SS}}$ : Negative supply voltage

(b) Gain $=0.8$

FIGURE 14: Example of signals obtained according to amplitude-to-time conversion method for two gain conditions: (a) 0.2 ; (b) 0.8.

In a complementary way, bioimpedance measurements in multiple locations of the biological medium can be combined to improve the sensitivity and specificity, as in EIT [26]. In this case, frequency- or time-division multiplexing can be used to avoid superposition in the excitation signals [131].

Some commercial chips have also been developed to perform bioimpedance measurements. AD5933 impedance network analyzer of Analog Devices performs impedance measurements between two terminals within the frequency range of $1 \mathrm{kHz}$ to $100 \mathrm{kHz}[22,95,108]$. For this, it uses a 12-bit ADC and a processing based on FFT. To allow the use of AD5933 in humans in a four-electrode configuration, analog-front-end (AFE) designs have been proposed [108, 132]. AFE 4300 of Texas Instruments is another example of 
a chip for bioimpedance measurements. Its design is based on a 6-bit pattern generator, a VCCS that generates a current signal between two terminals and a 16-bit ADC. However, the $150 \mathrm{kHz}$ limit of the system makes it unsuitable for bioimpedance spectroscopy applications [133]. AD8302 of Analog Devices is a phase and gain detector that has also been used in bioimpedance estimations [105]. These advances and the important research effort carried out in this field have allowed the development of wearable bioimpedance devices, some of which include wireless communication capabilities $[22,95,108,134]$.

2.6. Sources of Artifacts and Noise in Bioimpedance Measurements. A bioimpedance measurement system is more robust and stable against noise and motion artifacts than other biopotential measurement systems such as the ECG as a consequence of the injection of a reference current in one or more well-determined frequencies, which generates a voltage easily measurable in most cases [135]. However, bioimpedance measurements can be affected by multiple sources of error that can affect the signal-to-noise ratio (SNR) [22].

The first challenge faced by a bioimpedance device is the design of the current generator, which ideally must be precise and stable, both in modulus and phase, independently of the measured bioimpedance value [31]. This avoids the need for constant measurement of the injected current and minimizes its effect as a possible source of noise. A precise generation of a sine wave may require a high-precision DAC, which affects the power consumption and cost of the device [136]. In this case, a square signal can be used instead of a sinusoidal source. The harmonics generated by the square wave produce an error in the demodulation of the signal, but correction algorithms can be used to improve the precision of the estimations [136]. Another way to generate a precise sine signal is through a stable oscillator, as the case of the diode-stabilized Wien-Bridge oscillator described in [43]. To maintain the accuracy, the sinusoidal signal must be converted into current by means of a VCCS with a high output impedance and a high bandwidth, in order to support the bioimpedance variations without any distortion [43]. In the case of very small impedance, the gain of the VCCS can be controlled by increasing its value to improve the SNR [43], always within the safety limits established in IEC 60601 [95].

Another challenge is the design of a voltage measurement stage, sufficiently precise and robust against noise. It is common to use an instrumentation amplifier with a high input impedance, high common-mode rejection ratio (CMRR), typically greater than $80 \mathrm{~dB}$, and sufficient bandwidth to support the measurement frequencies without distortion [136-138]. However, the sensitivity requirements in the EIT applications are even stricter and any improvements provided in the accuracy of the measurements will result in a decrease in errors and an increase in the accuracy of the images. For this purpose, structures based on dual instrumentation amplifiers have been proposed to reduce the input noise, decrease energy consumption and improve the gain $[107,139$, 140].
In a system based on bioimpedance spectroscopy, the error in the estimates decreases if the number of measurement frequencies [133] increases and/or an exhaustive timing control of the signals is performed, as it can be done in a fieldprogrammable gate array (FPGA) device [106]. The sources of noise are also reduced by a suitable calibration of the bioimpedance device, incorporating the capacitive effects in the frequency response [141] and the effect of the electrodes [103]. This calibration is usually done manually, although it can be convenient to carry out automatically [22].

Some authors have shown the effect of the parasitic capacitance of the body to the earth $C_{B}$ as a major source of errors in bioimpedance measurements above $100 \mathrm{kHz}$ [142]. This capacitance can be from $11 \mathrm{pF}$ to $3.9 \mathrm{nF}$ for a standing person [143]. It produces gain and nonlinear errors whose main effects are the following $[142,143]$ :

(i) A gain error that increases with the frequency, behaving like an inductive effect proportional to the value of $\mathrm{C}_{\mathrm{B}}$ and the sum of all the resistances (measured and parasitic). This effect can even lead to positive phase angles in the bioimpedance measurement.

(ii) A gain error independent of the frequency and proportional to the ratio between $C_{B} / C_{C}$, where $C_{C}$ is the capacitance of the electrode. This effect translates into an offset in the magnitude of the bioimpedance.

(iii) A resonance effect that may be below $1 \mathrm{MHz}$, generated by the input capacitance of the instrumentation amplifier $\mathrm{C}_{\mathrm{in}}$ and the inductive effect of $\mathrm{C}_{\mathrm{B}}$. If $\mathrm{C}_{\mathrm{in}}$ is kept below $10 \mathrm{pF}$, it is possible to keep the resonance frequency above $10 \mathrm{MHz}$.

In bioimpedance systems based on spectroscopy, a key point is a correct identification of the single-dispersion Cole model parameters, since important errors can be made if this process is not carried out in a convenient way. As already mentioned in Section 2.3, the bioimpedance plot has a circular shape (see Figure 5). From the circular arc, the theoretical $\mathrm{R}_{0}$ and $\mathrm{R}_{\infty}$ can be approximated by projecting the curve that best fits the bioimpedance values [67]. The parameter $\alpha$ can be estimated taking into account the angle formed by the center of the circle with the real axis of the bioimpedance $\left(\varphi_{\mathrm{CPE}}=\alpha \pi / 2\right)$. The frequency at which the maximum of the absolute value of the bioimpedance imaginary part is produced corresponds to $f_{C}=1 / \tau$, where $\tau$ is the time constant of the dispersion. The value of this frequency allows the calculation of the parameter $\mathrm{C}$, since $\tau$ $=\left[\left(\mathrm{R}_{0}-\mathrm{R}_{\infty}\right) \mathrm{C}\right]^{1 / \alpha}$.

Cole model parameter identification is normally obtained by means of Nonlinear Least Squares (NLLS) methods, which aims at obtaining the best coefficients for the Cole model that fits the curve minimizing the summed squared of the error between the measured data and the modeled values [73, 144-146]. Some authors use only the impedance modulus when performing parameter identification [145, 147, 148], others only use the real or imaginary part [67]. However, the techniques that achieve a smaller error use both the real and the imaginary parts of the impedance [145] and recursive algorithms for parameter search, using NLLS methods [144]. 


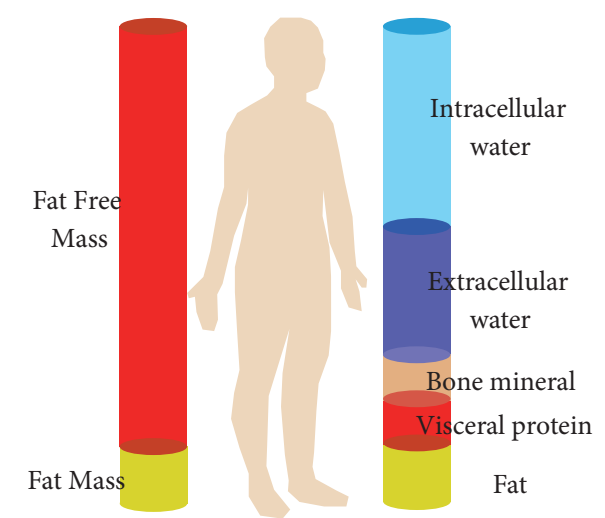

FIGURE 15: Compartmental model of the human body.

These methods do not often solve analytically the problem of parameter identification but use complex algorithms of successive approximations that can only be executed off-line on personal computers.

A major research effort has been made to reduce the amount of hardware needed and the cost of bioimpedance measurement instruments so that they can be used in portable applications $[149,150]$. Part of this effort has focused on reducing the processing costs so that it can be performed in portable devices [67].

\section{Bioimpedance for Body Composition Assessment}

The human body can be considered as a multicompartmental system, in which in a first instance each cell can be considered as a compartment [12]. In a generalized model, two compartments could be considered: the intracellular compartment, which contains the ICW, and the extracellular compartment, which integrates the ECW. The border between both media is the cell membrane [151]. The total body water (TBW) is the sum of ICW and ECW. The distribution of water content is nonuniform throughout the body and can be considered another model composed of a fat mass (FM) compartment, which includes water-free body lipids, and another compartment formed by the rest, called fat free mass (FFM) [151] (see Figure 15).

Bioelectrical impedance analysis (BIA) is a set of methods applied in the estimation of body composition through the measurement of bioimpedance [12]. The basis is the determination of the electrical impedance of an electric current that passes through the body that can be estimated in one or multiple frequencies. The BIA analysis has become a standardized technique in the estimation of body composition since it does not present the restrictions of the dissolution methods [152-154] and provides more accurate estimations than the anthropometric methods [154]. In addition, it is a simple, safe and noninvasive technique. Thanks to the BIA analysis it is possible to obtain an estimation of body fluid volumes and body composition in both normal and pathological states. The bioimpedance methods also have many practical advantages that have guided its rapid development
$[152,153]$ : the instrumentation is portable, relatively lowcost, and the measurements can be carried out quickly with minimal operator training; bioimpedance methods require little maintenance and the measurements are safe and easy to perform; it is a noninvasive technique, requiring only the placement of electrodes in the body; the results are obtained immediately, and the measurements can be repeated as often as desired, with great interobserver reproducibility.

The clinical usefulness of BIA techniques has been demonstrated in numerous studies: in nephrology $[12,155]$ (identification of dry weight in renal patients, improvement of cardiovascular management, monitoring of fluid transfer during ultrafiltration, estimation of the volume of distribution of urea in the calculation of the Kt/V parameter, or nutritional assessment), in nutrition (chronic malnutrition [156], obesity [157, 158], cachexia [159], sarcopenia [160], etc.), during pregnancy [161] and lactation [162], for the risk assessment of various pathologies [163], as a marker or direct cause of diseases [164], during the process of decision making in a disease, during aging or a rehabilitation process [165], as a complement in the diagnosis and monitoring of conditions related to the cardiovascular system (fluid accumulation after cardiac surgery [155], hyponatremia [166], etc.), in issues related to the immune system (patients with AIDS, dengue, hemorrhagic fever, and chronic inflammation [167]), in the evaluation of nutritional status in nervous system-related conditions (Alzheimer [155], anorexia nervosa [168], and mental disability [169]), in pediatrics [170], in oncology (evaluation of the patient's condition $[155,171]$, early diagnosis [172], etc.), in the postoperative period [171], for patients in critical care (follow-up of physiological trends in intensive care [173], sepsis [174], hemodynamic resuscitation [175], acute respiratory distress syndrome [176], patients bedridden [177], and patients with liver cirrhosis [178]), in gerontology $[179,180]$, and even in sports science (evaluation of the effectiveness of a training program [21], detection of anomalies in the distribution of liquids [181], etc.).

BIA analysis is also useful in chronic respiratory diseases, where the loss of body weight and the decrease in muscle mass have been recognized as risk factors associated with increased morbidity (inflammation, cachexia, anorexia, skeletal muscle dysfunction, increase of dyspnea, worsening of health status, increased risk of exacerbations, and decreased exercise capacity) [182], mortality [183], and a deterioration in the quality of life [184]. But fundamentally, BIA has a special relevance in patients with chronic or acute kidney disease, where fluid excess is also a condition related to increased morbidity and mortality [185].

The common basis of all BIA analysis systems is a measurement of the human body bioimpedance. To provide greater accuracy in estimations it is common to keep the human body in supine position for a time before the measurements, typically 15 minutes, to favor the balance of body fluids [186]. The electrodes are usually arranged according to the global lateral position shown in Figure 7, although many other configurations are possible [187]. Finally, the estimation of body composition is made from the analysis of bioimpedance measurements, which can be performed from 
TABLE 1: Examples of SFBIA equations.

\begin{tabular}{|c|c|c|}
\hline Compartment & Equation & Reference \\
\hline \multirow{2}{*}{ TBW } & Men: $1.02+0.449 \cdot \operatorname{Height}(\mathrm{cm})^{2} / \mathrm{R}_{50 \mathrm{kHz}}(\mathrm{ohm})+0.176 \cdot$ Weight $(\mathrm{kg})$ & \multirow{2}{*}{ [188] } \\
\hline & Women: $3.747+0.45 \cdot$ Height $(\mathrm{cm})^{2} / \mathrm{R}_{50 \mathrm{kHz}}(\mathrm{ohm})+0.113 \cdot$ Weight $(\mathrm{kg})$ & \\
\hline \multirow{2}{*}{ ECW } & Men: $-5.22+0.2 \cdot \operatorname{Height}(\mathrm{cm})^{2} / \mathrm{R}_{50 \mathrm{kHz}}(\mathrm{ohm})+0.005 \cdot \operatorname{Height}(\mathrm{cm})^{2} / \mathrm{X}_{50 \mathrm{kHz}}(\mathrm{ohm})+0.08 \cdot$ Weight $(\mathrm{kg})+1.9$ & \multirow{2}{*}{ [190] } \\
\hline & Women: ECW $(\mathrm{Men})+1.86$ & \\
\hline \multirow{2}{*}{ FFM } & Women: $-12.44+0.34 \cdot$ Height $(\mathrm{cm})^{2} / \mathrm{R}_{50 \mathrm{kHz}}(\mathrm{ohm})+0.1534 \cdot$ Height $(\mathrm{cm})+0.273 \cdot$ Weight $(\mathrm{kg})-0.127 \cdot$ Age $($ years $)$ & \multirow{2}{*}{ [191] } \\
\hline & Men: FFM (Women) +4.56 & \\
\hline \multirow{2}{*}{$\begin{array}{l}\text { Body fat } \\
\% \text { of the Weight }\end{array}$} & Women: $14.94-0.079 \cdot$ Height $(\mathrm{cm})^{2} / \mathrm{R}_{50 \mathrm{kHz}}(\mathrm{ohm})+0.818 \cdot$ Weight $(\mathrm{kg})-0.231 \cdot$ Height $(\mathrm{cm})+0.077 \cdot$ Age $($ years $)$ & \multirow{2}{*}{ [189] } \\
\hline & Men: Body Fat (Men) - 0.064·Weight $(\mathrm{kg})$ & \\
\hline
\end{tabular}

multiple approaches. The most representative methods are shown below.

(1) Single Frequency Bioimpedance Analysis (SFBIA). In order to simplify the analysis of bioimpedance, the human body can be modeled as a cylinder whose length is the height [66]. The electrical resistance of this model responds to the following generic expression, where $\sigma$ is the cylinder conductivity:

$$
R=\frac{\text { Height }}{\sigma \cdot \text { Area }}=\frac{\text { Height }^{2}}{\sigma \cdot \text { Volume }}
$$

Multiplying the numerator and denominator by the height, it is possible to obtain a generic expression of the volume depending on the height and resistance of the virtual cylinder that models the human body [52]:

$$
\text { Volume }=\frac{\text { Height }^{2}}{\sigma \cdot R}
$$

This relationship is the basis of SFBIA for the estimation of body compartment volumes. Numerous equations have been proposed in the literature for the approximation to the body parameters using the SFBIA technique [188-191]. These equations are usually defined from a regression analysis on a study population using the parameter Height ${ }^{2} / \mathrm{R}$ as one of the independent variables. To take into account the anthropometric characteristics of the subject, other variables such as weight or sex can also be incorporated as independent variables. The dependent variable is the body parameter to be estimated, which can be the volume of water (ICW, ECW, or TBW), but also the corresponding weight of fat or lean mass. The reference of this parameter is obtained from the standard gold estimation method, usually dissolution methods [133]. The resulting equations usually respond to the following expression $[66,192]$ :

$$
\text { Volume }=K_{0}+K_{1} \cdot \text { Weight }+K_{2} \cdot \frac{\text { Height }^{2}}{R}
$$

in which the coefficients $\mathrm{K}_{\mathrm{i}}$ are determined by the multivariate linear regression analysis in the study population [133]. Normally, SFBIA equations use the resistance value at the frequency of $50 \mathrm{kHz}$ [193], since this is the standardized frequency. The Table 1 shows some examples of equations based on the SFBIA method for different body compartments.
These equations have been selected for their widespread use and a minor error obtained in the evaluation studies [194]. The estimation of body parameters can be performed for a body segment (leg, arm, trunk, etc.) [195], modeling this segment as a cylinder, or for the whole body, in which case the different sections are approximated by different cylinders [52], although it could be considered a single cylinder that weighs the influence of the different body sections [196].

If the equations use the impedance obtained in two or more frequencies, the method is then called Multifrequency Bioimpedance Analysis (MFBIA). The main disadvantage of SFBIA and MFBIA methods is that the parameters of the equations are obtained empirically by means of a regression analysis. If these equations are applied in a different population group from which they were established, in altered states of composition or in people with anatomical particularities, important errors may occur in the estimations.

(2) Bioimpedance Spectroscopy (BIS). The BIS method removes the apparent population specificity of the SFBIA methods. This method is based on the fact that low-frequency currents only flow through the extracellular compartment, while high frequency currents circulate through both compartments. This assumption, together with the theory of mixtures of Hanai, has resulted in a set of equations for the estimation of the body compartments [63]. The ECW is obtained from the resistance $\mathrm{R}_{\mathrm{ECW}}=\mathrm{R}_{0}$ according to the following expression $[12,63,103,106,133]$ :

$$
E C W=\frac{1}{100} \cdot\left(\frac{K_{B \cdot} \rho_{E C W} \cdot \text { Height }^{2} \cdot \sqrt{\text { Weight }}}{D_{B}^{1 / 2} \cdot R_{E C W}}\right)^{2 / 3}
$$

in which $K_{B}$ is a dimensionless form factor with a typical value of 4.3 that incorporates the geometry of arms and trunk in the virtual cylindrical model of the human body [196], $\rho_{\mathrm{ECW}}$ is the resistivity of the extracellular medium, height is in $\mathrm{cm}$, Weight is in $\mathrm{kg}$, and $\mathrm{D}_{\mathrm{B}}$ is the body density in $\mathrm{kg} / \mathrm{L}$.

Similarly, TBW can be estimated from $\mathrm{R}_{\infty}$ by the following equation $[12,133,192,197,198]$ :

$$
T B W=\frac{1}{100} \cdot\left(\frac{K_{B} \rho_{\infty} \cdot \text { Height }^{2} \cdot \sqrt{\text { Weight }}}{D_{B}{ }^{1 / 2} \cdot R_{\infty}}\right)^{2 / 3}
$$

where $\rho_{\infty}$ is the expected resistivity of the human body at an infinite frequency. 


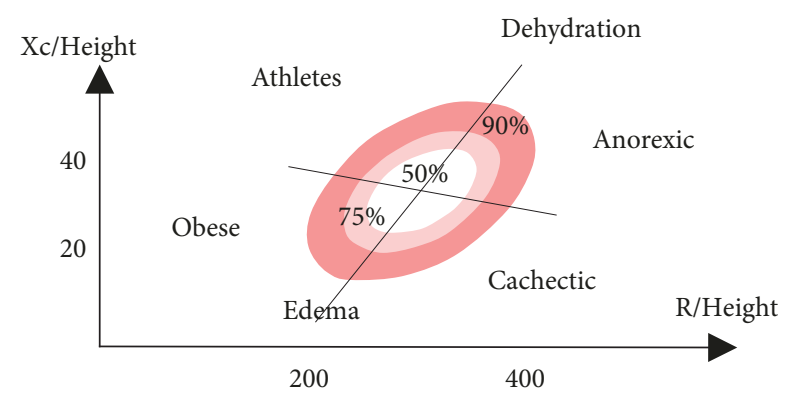

FIGURE 16: BIVA graph.

From TBW it is possible to calculate ICW, since ICW=TBW-ECW, and FFM, taking into account the fact that the water proportion of FFM in normal states is $73.2 \%$ [199]. In addition, the FM and its percentage can be established from FFM, since according to its own definition, FM=WFFM.

\section{Impedance Cardiography}

(3) Bioelectrical Impedance Vector Analysis (BIVA). The BIVA method does not require assumptions about geometry, as in the case of the BIS method, and it does not depend on approximations obtained by regression, as in the SFBIA method $[12,108,200]$. BIVA is based on a vector representation of the resistance and the reactance of the bioimpedance, normally at the frequency of $50 \mathrm{kHz}$, in absolute and normalized values with respect to the height of the subject. $\mathrm{R} /$ height is plotted on the abscissa axis and $\mathrm{X}_{\mathrm{C}}$ /Height on the ordinate axis [201]. Due to the correlation that exists between $R$ and $X_{C}$, the distribution of bioimpedances on the BIVA graph takes an elliptical form as shown in Figure 16. In this figure we can see the tolerance or reference ellipses that represent 50\%, 75\% and $95 \%$ of the reference population. Given their dependence on the study population, multiple reference ellipses have been proposed for different ethnic groups based on sex, age, and even body mass index (BMI) [108, 202, 203]. The bioimpedance values that fall outside the reference ellipse of $75 \%$ indicate an abnormal physiological situation, and depending on its location, the following analysis can be performed [12, 203]:

(i) Variations along the major axis of the ellipse are related to the state of hydration. Situations below the lower vertex indicate a state of overhydration or edema, or a state of dehydration above the opposite vertex.

(ii) Variations along the minor axis are associated with nutritional status, with excess of soft tissue or cells in situations that surpass the left vertex and deficit of cell mass with respect to the opposite vertex.

(4) Clinical Results in Body Composition Assessment. The estimations performed by bioimpedance devices have been compared with the results obtained by the methods considered gold standard in the measurement of the body composition (deuterium oxide dilution $\left(\mathrm{D}_{2} \mathrm{O}\right)$ or tritium dilution $(\mathrm{TrD})$ for TBW measurement, bromide dilution (BrD) for ECW assessment, total body potassium (TBK) for ICW determination and dual-energy X-ray absorptiometry (DEXA) for measurements of fat mass and lean mass). Tables 2 and 3 show some comparisons of the results obtained in several recent clinical studies. Table 2 shows the correlation coefficient $r$ and the $p$-value of statistical significance obtained in different correlation studies between the bioimpedance methods and the reference methods. Table 3 indicates the results of Bland Altman's analysis in relation to the mean value of the differences (reference value-BIA value) between the two estimations and the $95 \%$ confidence interval (CoIn) of the differences.

The comparative results of BIA methods show a good correlation and concordance with the reference methods, indicating an adequate accuracy. The BIS method is more accurate than the SFBIA method if the mean error is taken into account, although both ones are comparable. Usually, FFM is overestimated in BIA with respect to BIS. In contrast, fat mass is underestimated. Fluid excess can reduce the accuracy of DEXA, so bioimpedance may be a better option for patients in altered states of hydration. Moreover, DEXA which possibly underestimates FFM, which agrees with the estimates made by bioimpedance [204], does not allow evaluating the distribution of TBW between ECW and ICW so as to estimate body cell mass [205] and BIA has the advantage over DEXA of avoiding radiation exposure. As the BIA method is simpler, less invasive, and less expensive, its routine clinical use is reasonable [206]. Most studies on BIA also show its usefulness in the evaluation of changes in body composition [207].

Impedance cardiography (ICG) comprises a set of methods that provide an assessment of the transthoracic electrical bioimpedance and its time dependence on blood volume, circulation, and cardiac function [215]. It is also known as transthoracic electrical impedance plethysmography and provides a noninvasive approach to the measurement of the cardiac output [216]. Transthoracic impedance is modulated by cardiorespiratory activity, such that a decrease in impedance can be related to an increase of blood flow. The derivative of impedance $(\mathrm{dZ} / \mathrm{dt})$ contains a set of seven points (A, B, E, X, Y, O, Z, see Figure 17) related to cardiodynamic events [217]. Hence, cardiovascular condition and diverse hemodynamic parameters such as heart rate, cardiac volume, stroke volume, cardiac output, vascular resistance, velocity index, or thoracic fluid content, among others, can be noninvasively monitored against time by extracting characteristic points and periods of the dZ/dt signal through ICG [218]. Stroke volume (SV) can be estimated in milliliters per beat from Kubicek formula:

$$
S V=\left.\rho_{b}\left(\frac{L}{Z_{0}}\right)^{2} \cdot L V E T \cdot \frac{d Z}{d t}\right|_{\max }
$$

where $\rho_{\mathrm{b}}$ is the resistivity of blood, $\mathrm{L}$ is the transthoracic length, $Z_{0}$ is the impedance baseline, LVET is the leftventricular ejection time (i.e., $X-B$ segment), and $\left.(\mathrm{dZ} / \mathrm{dt})\right|_{\max }$ is the maximum slope of the impedance signal on a given beat. Cardiac output (CO) is computed in milliliters per 
TABLE 2: Comparison of correlation analysis results in studies of body composition.

\begin{tabular}{|c|c|c|c|c|c|c|c|}
\hline Study & Sample size & Measurement & $\begin{array}{c}\text { Reference } \\
\text { method }\end{array}$ & $\begin{array}{c}\text { Bioimpedance } \\
\text { method }\end{array}$ & Bioimpedance device & $\begin{array}{l}\text { Correlation } \\
\text { coefficient } \mathrm{r}\end{array}$ & $\mathrm{p}$-value \\
\hline \multirow{6}{*}{ [193] } & \multirow{6}{*}{$\begin{array}{l}94 \text { patients on } \\
\text { growth hormone } \\
\text { replacement } \\
\text { therapy }\end{array}$} & \multirow{6}{*}{ TBW (L) } & \multirow{6}{*}{$\operatorname{TrD}$} & SFBIA [208] & \multirow{6}{*}{$\begin{array}{c}\text { Body Scout, Fresenius } \\
\text { Medical Care, } \\
\text { Germany }\end{array}$} & 0.9 & \multirow{6}{*}{$\mathrm{p}<0.001$} \\
\hline & & & & SFBIA [189] & & 0.91 & \\
\hline & & & & SFBIA [209] & & 0.97 & \\
\hline & & & & SFBIA [188] & & 0.97 & \\
\hline & & & & BIS [154] & & 0.97 & \\
\hline & & & & BIS [63] & & 0.96 & \\
\hline [207] & $\begin{array}{l}122 \text { adult } \\
\text { hemodialysis } \\
\text { patients }\end{array}$ & TBW (L) & DEXA & BIS & $\begin{array}{l}\text { Body Composition } \\
\text { Monitor, Fresenius } \\
\text { Medical Care, } \\
\text { Germany }\end{array}$ & 0.87 & $\mathrm{p}<0.001$ \\
\hline \multirow{2}{*}[204]{} & 84 postmenopausal & Fat mass (kg) & \multirow{2}{*}{ DEXA } & \multirow{2}{*}{ MFBIA } & \multirow{2}{*}{$\begin{array}{l}\text { BIA-ACC, BIOTEKNA, } \\
\text { Italy }\end{array}$} & 0.95 & \multirow{2}{*}{$\mathrm{p}<0.001$} \\
\hline & women & Fat mass (\%) & & & & 0.76 & \\
\hline \multirow[t]{2}{*}[205]{} & $\begin{array}{l}11 \text { hemodialysis } \\
\text { patients }\end{array}$ & \multirow[t]{2}{*}{ Fat mass $(\mathrm{kg})$} & \multirow[t]{2}{*}{ DEXA } & \multirow[t]{2}{*}{ BIS } & \multirow{2}{*}{$\begin{array}{l}\text { Impedimed IMP SFB7, } \\
\text { Impedimed, USA }\end{array}$} & 0.96 & \multirow[t]{2}{*}{$\mathrm{p}<0.001$} \\
\hline & 25 healthy subjects & & & & & 0.92 & \\
\hline \multirow{2}{*}[210]{} & \multirow{2}{*}{30 healthy adults } & FFM (kg) & \multirow{2}{*}{ DEXA } & \multirow{2}{*}{ SFBIA } & \multirow{2}{*}{$\begin{array}{l}\text { RJL Systems Quantum } \\
\text { IV, RJL Systems, USA }\end{array}$} & 0.96 & \multirow{2}{*}{$\mathrm{p}<0.001$} \\
\hline & & Fat mass (kg) & & & & 0.92 & \\
\hline \multirow{3}{*}{ [206] } & \multirow{3}{*}{$\begin{array}{l}53 \text { adult } \\
\text { hemodialysis } \\
\text { patients }\end{array}$} & $\begin{array}{c}\text { Bone mineral } \\
(\mathrm{kg})\end{array}$ & \multirow{3}{*}{ DEXA } & \multirow{3}{*}{ SFBIA } & \multirow{3}{*}{$\begin{array}{l}\text { InBody S10, InBody, } \\
\text { Korea }\end{array}$} & 0.67 & \multirow{3}{*}{$\mathrm{p}<0.001$} \\
\hline & & Fat mass (kg) & & & & 0.93 & \\
\hline & & FFM $(\mathrm{kg})$ & & & & 0.96 & \\
\hline
\end{tabular}

minute as stroke volume times the heart rate. Cardiac index (CI) is obtained from $\mathrm{CO}$ normalizing with respect to body surface area.

Research works about ICG started in the 1940s and technology development in the 1960s. ICG bioimpedance is performed by using a constant current injection approach using a four-electrode (tetrapolar) arrangement. Usually, currents in the frequency range of $20-100 \mathrm{kHz}$ are used for excitation at the driving electrodes and voltage is measured at the sensing electrodes. The common configuration places the driving electrodes around the abdomen and the upper part of the neck, respectively, while the sensing electrodes are fixed around the thorax and the lower part of the neck [219]. Both band and spot electrodes have been reported for the use of ICG [220].

The advances in microprocessor technology and wireless communications have accelerated the design and development of wearable multifunction sensors providing the joint electrical (ECG) and mechanical (ICG) ambulatory monitoring of heart function using high-resolution analog-todigital converters [221, 222], embedded design with LabView [223], Raspberry PI platform [22], or off-the-shelf impedance converters [224]. The imperative of portability is a usual requirement for recent ICG monitors $[225,226]$. The use of textile electrodes has improved the portability of ICG systems through its seamless introduction in shirts [227], vests [228], the use of necklace-shaped electrode arrangements [229], or the adoption of contactless approaches [230, 231]. In other works, ICG and ECG are complemented with transmission and reflection photoplethysmography (PPG) to reduce the

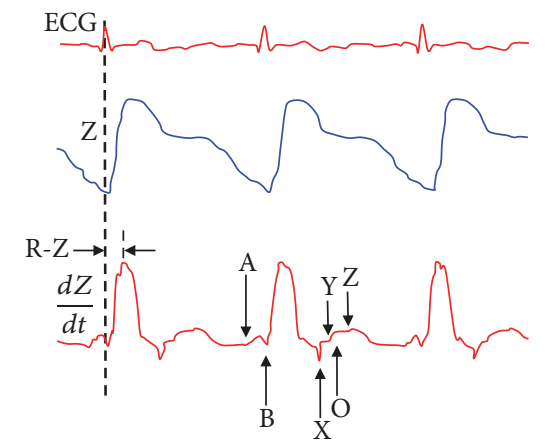

FIGURE 17: Transthoracic bioimpedance signal, its derivative, and relevant points (adapted from [220]).

sensibility of ICG to noise interference [232]. This interference has also been studied in [233] in the context of simultaneous functional magnetic resonance imaging and ICG acquisition, showing that changes in impedance $(\mathrm{dZ} / \mathrm{dt})$ are not affected.

In the context of processing the ICG signal, efforts are placed on improving the accuracy of slope change for the impedance derivative's $(\mathrm{dZ} / \mathrm{dt})$, which is related to the opening of the aortic valve [234]. The same issue has been addressed in [235] by considering the computation of the second and third derivatives, revealing the importance of the latter. The removal of ICG artifacts (both physiological and nonphysiological) has also been addressed by using adaptive filtering techniques [236] and wavelet analysis [237, 238]. 
TABLE 3: Comparison of bland Altman analysis results in studies of body composition.

\begin{tabular}{|c|c|c|c|c|c|c|c|}
\hline Study & Sample size & Measurement & $\begin{array}{c}\text { Reference } \\
\text { method }\end{array}$ & $\begin{array}{c}\text { Bioimpedance } \\
\text { method }\end{array}$ & Bioimpedance device & $\begin{array}{l}\text { Differences } \\
\text { Mean value } \\
\end{array}$ & $\begin{array}{l}95 \% \\
\text { CoIn } \\
\end{array}$ \\
\hline \multirow{6}{*}{ [211] } & \multirow{6}{*}{$\begin{array}{l}49 \text { hemodialysis } \\
\text { patients }\end{array}$} & TBW (L) & $\mathrm{D}_{2} \mathrm{O}$ & \multirow{3}{*}{ SFBIA } & \multirow{6}{*}{$\begin{array}{c}\text { Xitron 4200, Xitron } \\
\text { Technologies, USA }\end{array}$} & -2.1 & $\begin{array}{c}-5.3 \text { to } \\
1.1\end{array}$ \\
\hline & & ICW (L) & TBK & & & 0.5 & $\begin{array}{c}-1.5 \text { to } \\
2.4\end{array}$ \\
\hline & & ECW (L) & $\mathrm{BrD}$ & & & -4.4 & $\begin{array}{l}-6.2 \text { to } \\
-2.6\end{array}$ \\
\hline & & TBW (L) & $\mathrm{D}_{2} \mathrm{O}$ & \multirow{3}{*}{ BIS } & & 1.9 & $\begin{array}{c}-1.3 \text { to } \\
5.1\end{array}$ \\
\hline & & ICW (L) & TBK & & & 0.3 & $\begin{array}{c}-1.7 \text { to } \\
2.2\end{array}$ \\
\hline & & ECW (L) & $\mathrm{BrD}$ & & & -0.2 & $\begin{array}{c}-1.9 \text { to } \\
1.6\end{array}$ \\
\hline \multirow{6}{*}{ [193] } & \multirow{6}{*}{$\begin{array}{l}94 \text { patients on growth } \\
\text { hormone replacement } \\
\text { therapy }\end{array}$} & \multirow{6}{*}{ TBW (L) } & \multirow{6}{*}{$\operatorname{TrD}$} & SFBIA [208] & \multirow{6}{*}{$\begin{array}{l}\text { Body Scout, Fresenius } \\
\text { Medical Care, Germany }\end{array}$} & 2.3 & $\begin{array}{l}-5.8 \text { to } \\
10.4\end{array}$ \\
\hline & & & & SFBIA [189] & & -0.9 & $\begin{array}{c}-9.3 \text { to } \\
7.5\end{array}$ \\
\hline & & & & SFBIA [209] & & -2.7 & $\begin{array}{c}-7.5 \text { to } \\
2.1\end{array}$ \\
\hline & & & & SFBIA [188] & & -2.1 & -7.2 to 3 \\
\hline & & & & BIS [154] & & 0.6 & $\begin{array}{c}-4.2 \text { to } \\
5.4\end{array}$ \\
\hline & & & & BIS [63] & & 0.9 & $\begin{array}{c}-4.1 \text { to } \\
5.8 \\
\end{array}$ \\
\hline \multirow{3}{*}[212]{} & \multirow{3}{*}{$\begin{array}{l}130 \text { women with a } \\
\text { normal weight to the } \\
\text { third degree of obesity }\end{array}$} & \multirow{3}{*}{ Fat mass (kg) } & \multirow{3}{*}{ DEXA } & \multirow{3}{*}{ SFBIA } & $\begin{array}{l}\text { Bodystat } 1500, \text { Bodystat, } \\
\text { Isle of Man }\end{array}$ & -0.7 & -9.4 to 8 \\
\hline & & & & & $\begin{array}{c}\text { Omron BF 300, Omron, } \\
\text { Japan }\end{array}$ & -0.6 & -7.2 to 6 \\
\hline & & & & & $\begin{array}{c}\text { Tanita TBF } 410 \text { GS, } \\
\text { Tanita, Japan } \\
\end{array}$ & 0.6 & $\begin{array}{c}-7.3 \text { to } \\
8.5 \\
\end{array}$ \\
\hline \multirow{2}{*}[213]{} & \multirow{2}{*}{$\begin{array}{l}120 \text { pre-dialysis } \\
\text { patients }\end{array}$} & & \multirow[b]{2}{*}{ DEXA } & \multirow[b]{2}{*}{ BIS } & Body Composition & 3.1 & $\begin{array}{c}-6.8 \text { to } \\
13\end{array}$ \\
\hline & & FFM $(\mathrm{kg})$ & & & $\begin{array}{l}\text { Monitor, Fresenıus } \\
\text { Medical Care, Germany }\end{array}$ & -2.8 & $\begin{array}{l}-12 \text { to } \\
6.5\end{array}$ \\
\hline \multirow{4}{*}[204]{} & \multirow{4}{*}{$\begin{array}{l}84 \text { postmenopausal } \\
\text { women }\end{array}$} & Fat mass (kg) & \multirow{4}{*}{ DEXA } & \multirow{4}{*}{ MFBIA } & \multirow{4}{*}{$\begin{array}{l}\text { BIA-ACC, BIOTEKNA, } \\
\text { Italy }\end{array}$} & 3.5 & $\begin{array}{l}1.5 \text { to } \\
8.6\end{array}$ \\
\hline & & & & & & 5.3 & $\begin{array}{c}-2.3 \text { to } \\
12.8\end{array}$ \\
\hline & & FFM (kg) & & & & -2.9 & $\begin{array}{l}-11.1 \text { to } \\
5.4\end{array}$ \\
\hline & & FFM (\%) & & & & -5.2 & $\begin{array}{c}-12.5 \text { to } \\
2.1 \\
\end{array}$ \\
\hline [207] & $\begin{array}{l}122 \text { adult } \\
\text { hemodialysis patients }\end{array}$ & Fat mass $(\mathrm{kg})$ & DEXA & BIS & $\begin{array}{c}\text { Body Composition } \\
\text { Monitor, Fresenius } \\
\text { Medical Care, Germany }\end{array}$ & 0.3 & $\begin{array}{c}-5.5 \text { to } \\
6.2\end{array}$ \\
\hline \multirow[t]{2}{*}{ [210] } & \multirow[t]{2}{*}{30 healthy adults } & FFM (kg) & \multirow[t]{2}{*}{ DEXA } & \multirow[t]{2}{*}{ SFBIA } & \multirow{2}{*}{$\begin{array}{l}\text { RJL Systems Quantum } \\
\text { IV, RJL Systems, USA }\end{array}$} & -3.1 & $\begin{array}{l}-9.3 \text { to } \\
2.6\end{array}$ \\
\hline & & Fat mass $(\mathrm{kg})$ & & & & 4.2 & $\begin{array}{l}-5 \text { to } \\
12.7\end{array}$ \\
\hline$[214]$ & 31 healthy women & Fat mass (\%) & DEXA & SFBIA & BIA 101, Akern, Italy & 0.5 & $\begin{array}{c}-1.2 \text { to } \\
1.7\end{array}$ \\
\hline \multirow{3}{*}{ [206] } & \multirow{3}{*}{$\begin{array}{l}53 \text { adult hemodialysis } \\
\text { patients }\end{array}$} & $\begin{array}{c}\text { Bone mineral } \\
(\mathrm{kg})\end{array}$ & & & & 1.9 & $\begin{array}{l}0.9 \text { to } \\
2.9\end{array}$ \\
\hline & & Fat mass (kg) & DEXA & SFBIA & $\begin{array}{c}\text { InBody S10, InBody, } \\
\text { Korea }\end{array}$ & -6.5 & -15 to 2 \\
\hline & & FFM (kg) & & & & 4.2 & $\begin{array}{l}-3 \text { to } \\
11.5\end{array}$ \\
\hline
\end{tabular}


TABLE 4: Comparison of bland Altman analysis results in studies of ICG-based cardiovascular parameters.

\begin{tabular}{|c|c|c|c|c|c|c|}
\hline Study & Sample size & Measurement & Reference method & ICG device & $\begin{array}{l}\text { Differences } \\
\text { Mean value } \\
\end{array}$ & 95\% CoIn \\
\hline$[242]$ & $\begin{array}{c}42 \text { patients } \\
\text { (newborn - } 16 \text { yr) }\end{array}$ & $\mathrm{CI}\left(\mathrm{L} / \mathrm{min} / \mathrm{m}^{2}\right)$ & $\begin{array}{c}\text { Doppler } \\
\text { echocardiography }\end{array}$ & $\begin{array}{c}\text { NiCaS, NI Medical, } \\
\text { Israel }\end{array}$ & 0.05 & -0.77 to 0.87 \\
\hline$[243]$ & $\begin{array}{l}25 \text { healthy adults } \\
32 \text { patients with } \\
\text { chronic anemia }\end{array}$ & $\mathrm{CI}\left(\mathrm{L} / \mathrm{min} / \mathrm{m}^{2}\right)$ & $\begin{array}{c}\text { Magnetic } \\
\text { resonance imaging } \\
(\mathrm{MRI})\end{array}$ & $\begin{array}{l}\text { FhysioFlow, Manatec } \\
\text { Biomedical, France }\end{array}$ & $\begin{array}{l}0.1 \\
1.5\end{array}$ & $\begin{array}{l}-1.1 \text { to } 1.3 \\
-0.6 \text { to } 3.6\end{array}$ \\
\hline$[244]$ & 11 healthy adults & $\mathrm{SV}(\mathrm{mL})$ & MRI & $\begin{array}{c}\text { Cardiotronic, Osypka } \\
\text { Medical, Germany }\end{array}$ & $\sim 0$ & -64 to 64 \\
\hline$[245]$ & $\begin{array}{c}17 \text { patients under } \\
\text { hemodialysis }\end{array}$ & $\mathrm{SV}(\mathrm{mL})$ & $\begin{array}{c}\text { Doppler } \\
\text { echocardiography }\end{array}$ & $\begin{array}{c}\text { NiCaS, NI Medical, } \\
\text { Israel }\end{array}$ & 1.3 & -11.6 to 14.1 \\
\hline \multirow{2}{*}[246]{} & \multirow{2}{*}{32 healthy adults } & $\mathrm{SV}(\mathrm{mL})$ & Doppler & \multirow{2}{*}{ non-commercial } & -1.53 & -26.7 to 23.64 \\
\hline & & $\mathrm{CO}(\mathrm{L} / \mathrm{min})$ & echocardiography & & 0.32 & -1.19 to 0.18 \\
\hline \multirow{2}{*}[247]{} & \multirow{2}{*}{$\begin{array}{l}53 \text { pregnant } \\
\text { women }\end{array}$} & SV (mL) & Doppler & \multirow{2}{*}{$\begin{array}{l}\text { ICG, Philips Medical } \\
\text { Systems, USA }\end{array}$} & 2.5 & -15.7 to 20.7 \\
\hline & & $\mathrm{CO}(\mathrm{L} / \mathrm{min})$ & echocardiography & & 0.2 & -1.3 to 1.7 \\
\hline
\end{tabular}

The use of artificial neural networks has been proposed to improve the calculation of stroke volume from ensemble averages of ICG signals [239]. Finally, kernel methods have been used to analyze the time-frequency content of the ICG signal in [240, 241].

While some studies have warned against the accuracy of ICG-based measurements compared with gold standards for cardiovascular condition (Table 4 summarizes recent studies for diverse health conditions), its low-cost and ease of use make this technique appropriate for clinical use.

\section{Transthoracic Impedance Pneumography}

Transthoracic impedance pneumography (TIP) is a noninvasive method that evaluates the thoracic impedance and changes related to respiratory activity, becoming an alternative to spirometry or pneumotachometry for ambulatory and outside hospital settings [248]. It offers a simple way for the characterization of respiration rate together with other vital pulmonary signs, such as respiration cycle length and tidal volume. Hence, it provides essential indicators for the respiratory condition. Breathing activity changes the thoracic volume, geometry, and conductivity, thus modulating the impedance that can be recorded between two surface electrodes placed on the chest [249]. While it can be acquired from the same electrodes as ECG [250], TIP uses $50-500 \mathrm{kHz}$ low amplitude currents. Tetrapolar electrode configuration is also popular to reduce skin-electrode effects [251]. Due to the conductivity of lung tissue, most of the impedance changes are due to the current passing through the chest and back. Upon respiration activity, voltage increase and drop are associated with lung air filling (inspiration) and emptying (expiration), respectively, with a linear correlation between impedance changes and the volume of ventilated air [252]. In [253], such relation is expressed similarly to (24) as

$$
\Delta V_{L}=\rho \cdot\left(\frac{L}{Z_{0}}\right)^{2} \cdot \Delta Z
$$

where $\mathrm{V}_{\mathrm{L}}$ and $\Delta \mathrm{Z}$ are the respiration volume change and magnitude of the impedance change, respectively. $\mathrm{L}$ is the length of the conducting volume, $\mathrm{Z}_{0}$ the impedance baseline, and $\rho$ a resistivity parameter. Flow can be expressed as

$$
\Phi=\frac{d V_{L}}{d t}
$$

However, the respiratory airflow monitored by TIP is sensitive to movement artifacts [254].

TIP has been used to measure lung volume changes [255], to evaluate respiratory condition during normal and deep breathing [256], the assessment of asthma risk from tidal flow variability [257], sleep disorders [258], energy expenditure estimation [259], or even the detection of body posture changes [260]. Another application of TIP is the gating of positron-emission tomography (PET) and singlephoton emission computed tomography (SPECT) images. Respiratory movement is an important source of error in studies using PET images of the thoracic region. Due to the long period of time necessary for taking a PET image, usually minutes, the respiratory movement can deteriorate the image quality [261], leading to an incorrect diagnosis or inadequate treatment [262]. TIP has been used to compensate for the adverse effects of movement in oncological PET images [261]. It has also been applied to reduce the effects of respiratory movements in the analysis of myocardial perfusion SPECT images [263].

Like ICG, the e-health paradigm has fostered the design of wearable sensors supported by signal processing algorithms. Among the former, textile electrodes have been designed in [264] to provide joint ECG and TIP monitoring. The careful design of the electrodes has stimulated research, with the adoption of textile multimaterial fibers for contactless TIP sensing [265], compound electrodes [266], and graphene electrodes for a long-term measurement life [267]. Signal processing methods for TIP are focused on the derivation of respiratory rate through autoregressive models [250] or power spectral density estimation [268], the decomposition of the cardiac and respiratory components [269], 
TABLE 5: Bland Altman analysis results obtained in TIP.

\begin{tabular}{|c|c|c|c|c|c|c|}
\hline Study & Sample size & Measurement & $\begin{array}{c}\text { Reference } \\
\text { method }\end{array}$ & TIP device & $\begin{array}{l}\text { Differences } \\
\text { Mean value }\end{array}$ & 95\% CoIn \\
\hline [271] & $\begin{array}{l}20 \text { adult patients in } \\
\text { postoperative care }\end{array}$ & $\begin{array}{l}\text { Respiratory rate } \\
\text { (breaths/min) }\end{array}$ & Capnography & $\begin{array}{c}\text { Datex Ohmeda } \\
\text { Compact S3 } \\
\text { (e-PRESTN), Datex } \\
\text { Ohmeda, Finland }\end{array}$ & -1.9 & -13.1 to 9.2 \\
\hline [272] & $\begin{array}{l}98 \text { patients } \\
\text { scheduled for } \\
\text { upper } \\
\text { gastrointestinal } \\
\text { endoscopy }\end{array}$ & $\begin{array}{l}\text { Respiratory rate } \\
\text { (breaths/min) }\end{array}$ & $\begin{array}{l}\text { Acoustic } \\
\text { respiratory rate } \\
\text { monitoring }\end{array}$ & $\begin{array}{l}\text { AG-920RA bedside } \\
\text { monitor, Nihon } \\
\text { Koden, Japan }\end{array}$ & 0.4 & -11.1 to 11.9 \\
\hline
\end{tabular}

the improvement of respiratory parameters using nonlinear corrections with neural networks [270], and the removal of motion artifacts based on correlation measurements [251].

Given the characteristics of the signal, TIP has been evaluated as an alternative in the study of the respiratory rate $[271,272]$. Table 5 shows the results obtained in two recent clinical studies. Another application of TIP is the gating of positron-emission tomography (PET) and singlephoton emission computed tomography (SPECT) images. Respiratory movement is an important source of error in studies using PET images of the thoracic region. Due to the long period of time necessary for taking a PET image, usually minutes, the respiratory movement can deteriorate the image quality [261], leading to an incorrect diagnosis or inadequate treatment [262]. TIP has been used to compensate for the adverse effects of movement in oncological PET images [261]. It has also been applied to reduce the effects of respiratory movements in the analysis of myocardial perfusion SPECT images [263].

\section{Electrical Impedance Tomography}

Introduced by Barber and Brown in the 1980s [273], electrical impedance tomography (EIT) is a medical imaging modality that provides the spatial distribution of bioimpedance inside the body [274], relying on a set of 16-32 electrodes [275] placed on the surface confining the volume of interest. Current excitation in the typical frequency range of 20$250 \mathrm{kHz}$ causes an electromagnetic field that interacts with volume tissues yielding electric potential maps that can be measured [276]. However, different operating frequencies have been proposed for EIT systems. For instance, the KHU Mark 2.5 EIT system, designed for imaging brain function, operates within $11-500 \mathrm{kHz}$ [277], and broadband EIT systems for breast imaging span from $10 \mathrm{kHz}$ to $12.5 \mathrm{MHz}$ [278]. A high output impedance current source is required for broadband EIT applications [279].

Two complementary problems may be distinguished in the context of EIT. The forward problem assumes a known conductivity distribution within volume tissues and involves the numerical computation of voltages on the surface electrodes [280]. The inverse problem addresses the prediction of conductivity distribution from the electric potential measurements through a reconstruction algorithm [281], from which bioimpedance images are derived. Among the advantages, EIT outstands as a low-cost, harmless, simple, and noninvasive technique yielding high-speed imaging and high temporal resolution. It can be very fast, able to generate thousands of images per second. Compared to nuclear imaging, EIT does not use ionizing radiations and thus harmful side effects are avoided. Its drawbacks are associated with the limited spatial resolution it provides [282] due to physical constraints for electrode separation and the numerical issues for impedance image reconstruction due to the ill-posed nature of the inverse problem. However, compared to other imaging modalities EIT is regarded as a high-resolution technique in terms of the functional parameters that it provides.

In the biomedical field, EIT has been applied to the examination of chest [283], to manage patients with acute respiratory distress syndrome (ARDS) in the intensive care unit (ICU) by estimating regional lung ventilation at the bedside [284]. Other areas of application include stroke imaging [285], the characterization of brain tissues [26, $286]$, the monitoring of regional cerebral edema during clinical dehydration treatment [287], the early warning of brain injury during aortic arch replacement operation [288], gesture recognition [289], cancer detection [290-292], pediatric intensive care [293], tissue engineering [294], thermal management of hyperthermia [295], or laparoscopic surgery [296]. Another application of EIT is the gating of positronemission tomography (PET) and single-photon emission computed tomography (SPECT) images. Respiratory movement is an important source of error in studies using PET images of the thoracic region. Due to the long period of time necessary for taking a PET image, usually minutes, the respiratory movement can deteriorate the image quality [261], leading to an incorrect diagnosis or inadequate treatment [262]. EIT has been used to compensate for the adverse effects of movement in oncological PET images [261]. It has also been applied to reduce the effects of respiratory movements in the analysis of myocardial perfusion SPECT images [263]. Other applications have been reviewed in [297].

The EIT forward problem usually relies on the finiteelement (FE) method. The dimensionality of the forward problem has been regarded as the most critical factor limiting the quality of image reconstruction [298]. The analysis of convergence of the FE approximation for EIT has been analyzed in [299], showing that the estimated voltages on the 
TABLE 6: Bland Altman analysis results obtained in EIT studies.

\begin{tabular}{ccccccc}
\hline Study & Sample size & Measurement & Reference method & EIT device & $\begin{array}{c}\text { Differences } \\
\text { Mean value }\end{array}$ & 95\% CoIn \\
\hline$[339]$ & $\begin{array}{c}\text { 14 patients with severe } \\
\text { respiratory failure }\end{array}$ & $\begin{array}{c}\text { Regional expiratory } \\
\text { time constant (s) }\end{array}$ & $\begin{array}{c}\text { Anemometry } \\
\text { (V-Meter, EKU, } \\
\text { Germany) }\end{array}$ & $\begin{array}{c}\text { Swistom BB2, } \\
\text { Swisstom, } \\
\text { Switzerland) }\end{array}$ & 0.14 & 0.37 to 0.65 \\
\hline [340] & $\begin{array}{c}\text { 15 ventilated intensive } \\
\text { care patients }\end{array}$ & $\begin{array}{c}\text { Positive } \\
\text { end-expiratory } \\
\text { pressure (PEEP) } \\
\text { (mbar) }\end{array}$ & PEEP trial & $\begin{array}{c}\text { Pulmovista 500, } \\
\text { Draguer, Germany }\end{array}$ & 1 & 2 to 1 \\
\hline [341] & 9 healthy piglets & Ventilation (ml) & $\begin{array}{c}\text { Vibration response } \\
\text { imaging }\end{array}$ & $\begin{array}{c}\text { Goe-MFII, } \\
\text { CareFusion, Germany }\end{array}$ & -1 & -49 to 47 \\
\hline
\end{tabular}

electrodes converge to the true values as the underlying mesh is more refined. Regarding the inverse problem, different linearization methods have been proposed to address the linear inverse in an iterative algorithm [300]. Among the regularization approaches for the nonlinear inverse problem, the "D-bar method" [301] and the "Calderón method" [302] are proven strategies which have been extended in [303] with a convolutional neural network to prevent image blurring. Deep learning approaches applied to image reconstruction for EIT have also been addressed in $[304,305]$. The incorporation of structural information has been applied in [306] to reduce the dimensionality and sensitivity to regularization parameters. Another way of compensating the ill-posedness of the inverse problem is the enforcement of monotonicity constraints about the reconstructed conductivity [307309]. In other papers, regularization incorporates a priori knowledge yielding sparse solutions [306]. Other works combine level set function, shape and topological derivatives to avoid local minima in the optimization algorithm [310]. The consideration of alternate solution spaces, such as the logarithmic conductivity, has proven to be more robust to the initial guess and yield faster convergence [311]. With regard to EIT image resolution, resampling-based methods [312] and sparse Bayesian learning [313] have been suggested to improve the quality of EIT image reconstruction. In other words, multiple information fusion methods are approached to address the limitations of EIT. Careful considerations of a priori information, measurement error, and the model parameters are required, together with close collaboration between mathematicians and experimentalists.

During the last couple of years, EIT sensors and systems have been customized for real-time operation and portability. A wearable EIT system has been proposed in [314] based on system-on-chip technology. It integrates 48 channels in a flexible printed circuit board belt with wireless communications to an external imaging device. Another belt design with 32 electrodes is reported in [294], which uses active electrode ASIC (Application-Specific Integrated Circuit) technology. The electronic sensing architecture proposed in [315] uses dry electrodes integrated in a vest for the simultaneous EIT and multilead ECG data acquisition through frequency division multiplexing. The prototype described in [316] uses an arrangement of 16 microelectrodes placed around the base of a circular chamber for $3 \mathrm{D}$ cultivation imaging. The system in [317] integrates multifrequency operation with 32 electrodes for $2 \mathrm{D}$ and $3 \mathrm{D}$ imaging. Alternative methods to sequential electrode excitation have also been tested in [318], which uses mixed excitation patterns yielding a simultaneous linear combination of single excitations. The reconfigurability and control of the variables involved in the experimental operation is another highly demanded requirement [319] for EIT systems.

As it has been previously mentioned, the medical images provided by EIT are of great clinical utility. These images have also been used to derive clinical parameters from the analysis of their evolution. Table 6 summarizes some examples of recent studies that compare estimates against reference standards.

\section{Skin Conductance Applications}

The sympathetic skin conductance response (SCR) is one index of autonomic arousal [320]. Electrodermal activity (EDA) measures the electrical activity on the surface of the skin or originating from the skin [321]. It can be characterized as a tonic level through skin conductance or as a transient response through galvanic skin response. EDA measurement systems are based on the injection of a signal-usually an electric current-into the body using surface electrodes attached to the skin, and the electric voltage developed as a consequence of the injection of the current is usually recorded. Sweat gland permeability is the main responsible for skin conductance changes [322]. As sweat gland activity is neurally mediated through sympathetic activity, EDA is highly appreciated in psychophysiology and behavioral medicine [323]. However, EDA is also influenced by factors such as external temperature, and requires reference measurements and calibration [324].

EDA processing is focused on activity modeling and the removal of artifacts. While exponential smoothing [325] and low-pass filtering [326] are common approaches to signal denoising, wavelet transform has been used in [327] for motion artifact reduction in drivers and machine learning algorithms have been suggested in [328] to distinguish artifacts in an EDA signal from normal physiological responses. Sparse recovery based on the orthogonal matching pursuit algorithm has been applied in [329] to separate skin conductance response from artifacts with a high accuracy. For 
TABLE 7: Bland Altman analysis results obtained in skin conductance studies.

\begin{tabular}{|c|c|c|c|c|c|c|}
\hline Study & Sample size & Measurement & Reference method & SCR device & $\begin{array}{l}\text { Differences } \\
\text { Mean value }\end{array}$ & 95\% CoIn \\
\hline \multirow{2}{*}{ [337] } & \multirow{2}{*}{49 healthy elderly } & $\begin{array}{l}\text { Resting energy expenditure } \\
\text { (kcal/24h) in the morning }\end{array}$ & \multirow{2}{*}{$\begin{array}{l}\text { Indirect } \\
\text { calorimetry }\end{array}$} & \multirow{2}{*}{$\begin{array}{l}\text { Sensewear armband, } \\
\text { Body- Media, USA }\end{array}$} & -166 & -438 to 105 \\
\hline & & $\begin{array}{l}\text { Resting energy expenditure } \\
\text { (kcal/24h) at night }\end{array}$ & & & -187 & -427 to 53 \\
\hline \multirow{2}{*}{ [338] } & \multirow{2}{*}{$\begin{array}{c}133 \text { type } 2 \text { diabetic } \\
\text { patients and } 41 \\
\text { control subjects }\end{array}$} & $\begin{array}{l}\text { Sudomotor function (\%) on } \\
\text { hands }\end{array}$ & \multirow{2}{*}{$\begin{array}{l}\text { Quantitative } \\
\text { sudomotor axon } \\
\text { reflex test }\end{array}$} & \multirow{2}{*}{$\begin{array}{l}\text { EZSCAN, Impeto } \\
\text { Medical, France }\end{array}$} & -4.3 & -23.5 to 15 \\
\hline & & $\begin{array}{l}\text { Sudomotor function (\%) on } \\
\text { foots }\end{array}$ & & & -1 & -14 to 12 \\
\hline
\end{tabular}

the analysis of EDA signals and identification of arousals, the methods range from power spectral density analysis [330, 331], machine learning [332], compressed sensing [333], to nonlinear analysis based on chaotic characterization and complexity assessment [334].

EDA sensors can be based on $\mathrm{Ag} / \mathrm{AgCl}$ disc electrodes attached to the palm sides of index and middle fingers, such as the prototype reported in [335], or multilayered sensor disc for use as a dry electrode [336]. The use of spiral geometry for the electrodes has been suggested in [38] to make measurement results independent of skin anisotropy.

An example of application of skin conductance measurement is the evaluation of the metabolic condition of a person. Table 7 shows the results obtained in a comparative study of the skin conductance with the measurement of the resting energy expenditure [337]. Another example is the evaluation of sudomotor function [338].

\section{Impedance Detection in Biosensors}

Impedance biosensors are in use for the detection and characterization of different bioparticles as bacteria, viruses, cells, and biomolecular structures by the aid of measurement of their electrical impedance in microfluidic environment. The rise of impedance-based biosensors was initiated by the following two enabling factors.

(1) Impedance sensing in electrochemistry: first, the impedance-based electrochemical sensors for the detection and concentration determination of chemical have been known for many decades already [342]. Electrochemical sensors have used the impedance measurement for a long time already [342], e.g., for determination the concentration of ionic reagents in solutions and for monitoring reduction-oxidation (redox) reactions. Emergence of electrical current as the product of chemical reactions is known as the Faradaic process. The level of evoked current depends on intensity of chemical reactions. Sensing methods are potentiometry, amperometry, and voltammetry using either direct current or slowly changing alternating current. Such the sensing method is called Faradaic, which are widely used in electrochemistry, but preferably not in biosensors. Electrochemistry gave impetus to the use of impedance sensing in biosensors, but the biosensors use mostly non-Faradaic formation of electrical current, which depends on the rate of presence (concentration) certain biological particlescells and biomolecules as DNA, but mostly the pathogens as bacteria and viruses-causing changes in the impedance to alternating electrical current in $\mathrm{kHz}$ and $\mathrm{MHz}$ frequency range.

(2) Bioimpedance sensing in microfluidics: another boost to the rise of impedance methods gave an expansion of microfluidic technology, enabling to create miniature lab-on-a-chip (LoC) devices [343]. First of all, the bioparticles were discovered and characterized by labeling-introducing certain chemicals for visual detection through the changing of label colour. Unfortunately, the labeling chemicals are not fully passive; they change the properties of fluids and can change also the bioparticles to be detected. Moreover, the labeling chemicals are not always available and can be expensive. Using of impedance methods enables the label-free detection of bioparticles. Besides, the label-free impedance detection is the fastest method known.

Both the above described reasons have worked as accelerators for introducing the methods for bioimpedance detection and analysis. Two types of impedance biosensors are known:

(1) Sensors of floating/flowing bioparticles: the task is to detect the floating or fast flowing bioparticles in a narrow submillimeter or micrometer range channel, only a slightly larger than detectable bioparticles. Such the devices are, for example, impedance cytometers intended for the counting and selection of single cells, e.g., for making difference between bigger and smaller cells or for separating the living and dead cells. Bare or coated (electrically insulated) metal electrodes are in use. Capturing of particles to electrodes is not requested, on the contrary, it should be avoided. Fast flow of fluids containing particles is needed [344].

(2) Affinity biosensors: the affinity biosensors selectively detect the bound (immobilized) bioparticles. At least one of the electrodes is covered by a selectively acting layer (antibody or aptamer layer), which binds to itself 


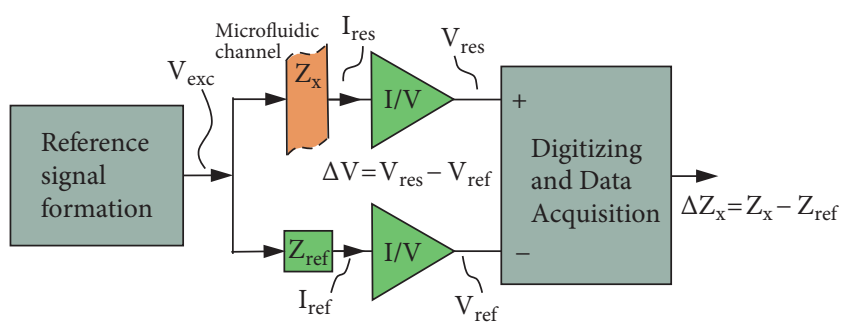

FIGURE 18: A generalized measurement scheme for differential measurement of changes $\Delta \mathrm{Z}_{\mathrm{x}}$ of the impedance to be measured $\mathrm{Z}_{\mathrm{x}}$. The current responses $\left(\mathrm{I}_{\text {res }}\right.$ and $\mathrm{I}_{\text {ref }}$ ) to voltage excitation $\mathrm{V}_{\text {exc }}$ are converted into response voltages $\left(\mathrm{V}_{\text {res }}\right.$ and $\left.\mathrm{V}_{\text {ref }}\right)$; the difference of which is amplified, acquired, and digitized by the aid of differential input analog-to-digital converter giving the digital output $\Delta \mathrm{Z}_{\mathrm{x}}$.

the specific bioparticles (bacteria and viruses, also big protein molecules), existing in the surrounding fluid. Antibodies exist in human and animal organisms as the guarantors of immunity or are formed there if the pathogens attack. As already told, the antibodies are selective, they bind only these pathogens (named as antigens, in general), which called them up. Producing of natural antibodies can be complicated and costly. Artificially synthesized aptamers have the same binding effect, but are cheaper and more flexible to modify, only their production technologies are still in development stage.

8.1. Impedance Measurement Principles in Biosensors. Important is to underline that it is extremely hard to measure tiny impedance deviations $\Delta Z_{x}$ around 0.05 to $0.5 \%$ from its basic value $Z_{x}$. Therefore, differential measurements are needed. In Figure 18, there is given a 2-channel measurement scheme to determine, how much the impedance to be measured $Z_{x}$ (impedance of the microfluidic channel in Figure 18) differs from the reference value $Z_{\text {ref }}$, which is introduced as an electrical equivalent circuit mimicking the measurement channel (microfluidic channel) physically. As a result, the resolution of measurement enhances at least tens and hundreds of times. In microscale biosensors, mostly the voltage excitation $\mathrm{V}_{\mathrm{exc}}$ and current response $\mathrm{I}_{\text {res }}$ are used for impedance measurement.

8.2. Biosensor Electrodes. The measurement of impedance $Z_{x}$ requires electrodes made from conductive material, frequently metallic ones or carbon based. In microfluidic channels, mostly golden electrodes are used for bioimpedance measurement, bare or covered by thin electrically insulating layer. Electrodes for affinity sensors use multilayer structure on gold (see Figure 21).

Though mostly quadrupole electrodes (4-electrode system, one pair for excitation current and the other one for voltage pick-up) are used for bioimpedance measurement, only two electrodes are predominantly applied both for the voltage excitation and current response. The reason is that it is complicated to take advantage of 4-electrode system in miniature non-Faradaic biosensors. It is important to remember that there is no current due to chemical reactions but solely due to the voltage excitation. The electrical current through the sensing part between electrodes is ionic (charge carriers are ions), but outside of this the charge carriers are electrons. As a result, a thin interfacial double layer appears at electrodes, which behaves like a modified capacitance and is modeled as CPE (see (27)). The impedance of CPE can be expressed through the frequency dependent magnitude $\mathrm{Z}_{\mathrm{CPE}}(\omega)$ and constant phase $\varphi$, where $\mathrm{n}$ can be set from $\mathrm{n}=0$ (ideal resistor $\mathrm{R}$ ) through $\mathrm{n}=0.5$ (ideal Warburg impedance) to $\mathrm{n}=1$ (ideal capacitor $\mathrm{C}$, valid in case of electrically insulated electrode):

$$
\mathrm{Z}_{C P E}(\omega)=\frac{Z_{0}}{\omega^{n}}
$$

and

$$
\phi=-n \frac{\pi}{2}
$$

The frequency response plot of the CPE at different values of $\mathrm{n}$ is given in Figure 19(b) in logarithmic scale.

Configurations of the most commonly used electrodes for miniature impedance biosensors are depicted in Figure 20 $[345,346]$. The simplest one in Figure 20(a) represents linearly placed quadrupole electrodes. A similar arrangement of concentric configuration is given in Figure 20(b). A linear configuration of 2-pole interdigitated electrodes covering a wider sensing area is given in Figure 20(c). Analogous interdigitated sensor with concentric arrangement of electrodes is given in Figure 20(d).

8.3. Affinity Biosensor. A typical affinity based biosensor for the bioimpedance detection of pathogen in a biological solutions which uses gold electrodes on substrate is depicted in Figure 21. To connect antibody (or aptamer) layer strongly onto gold electrode, a thin nanometer range self-assembling monolayer (SAM) is required for interfacing inorganic electrode from one side and the biological material (biomolecules, cells, liquid). Thiol and dithiol SAMs on metals, and particularly on gold, have attracted attention due to their easy preparation from gas phase or from solution, and their relatively high stability mediated by the strength of the SAu (sulfur-gold) bond and by van der Waals forces from molecular interactions [347]. If there exist appropriate bioparticles (named also as antigens) in saline solution, then the selective antibody layer selects these antigens (bacteria, viruses) and antigen-antibody layer will be formed. Antigens (pathogens) change the impedance of antibody layers. This change $\Delta Z_{x}$ informs us on the presence of corresponding pathogens and enables also to estimate their concentrations.

The multilayer coating on gold electrode can be divided into two parts. First, the complex impedance $Z_{e}(j \omega)$ takes in only the double layer and the accompanying phenomena (Figure 19(a)). The other part of impedance, $Z_{x}(j \omega)$, contains the impedance of SAM, antigen-antibody layer and saline solution with floating bioparticles $\left(\mathrm{R}_{1}, \mathrm{R}_{2}\right.$, and $\mathrm{C}_{1}$ together with a parasitic stray capacitor $C_{p}$ ); see Figure 19(a). Resistor $\mathrm{R}_{1}$ brings together the resistive components of saline solution, SAM, and antigen-antibody layer, changing the last of which 


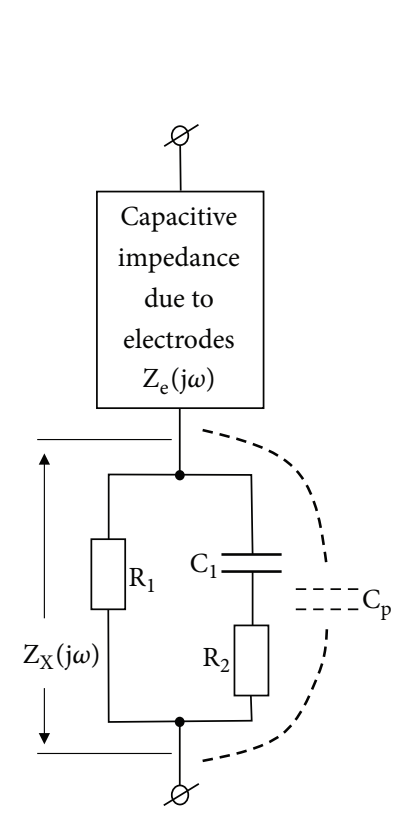

(a)

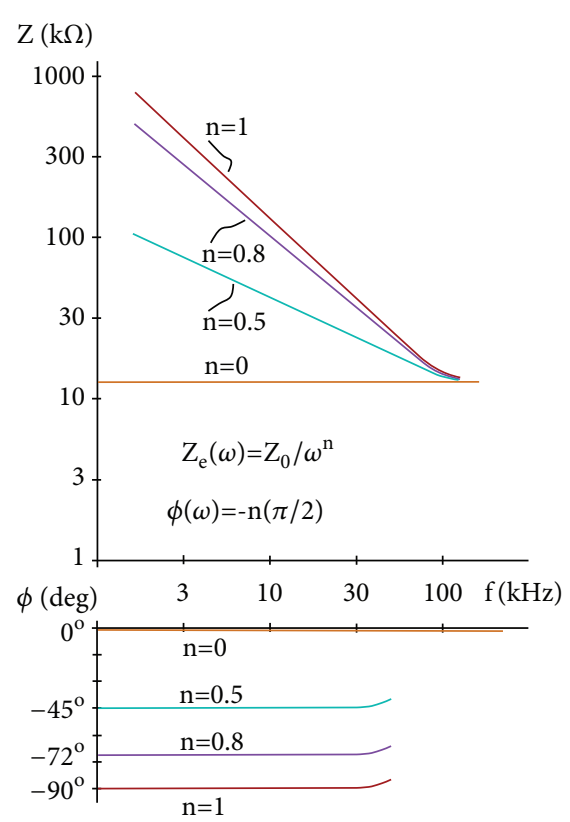

(b)

FIGURE 19: Characterization of non-Faradaic affinity based impedance biosensors: (a) an equivalent electrical circuit; (b) the frequency response of its impedance magnitude $\mathrm{Z}(\mathrm{f})$ and constant phase $\varphi$.

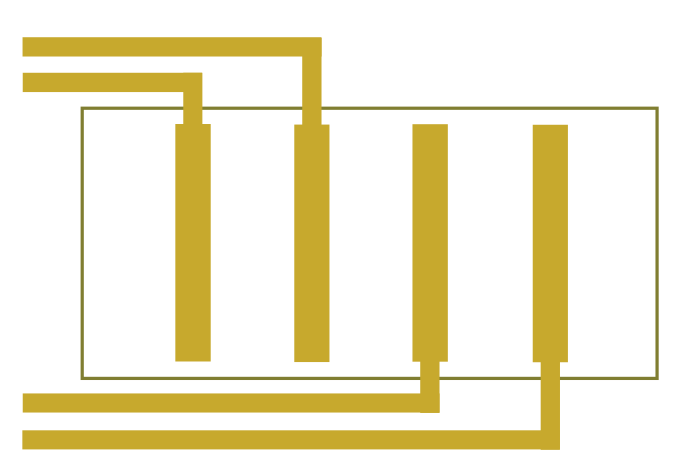

(a)

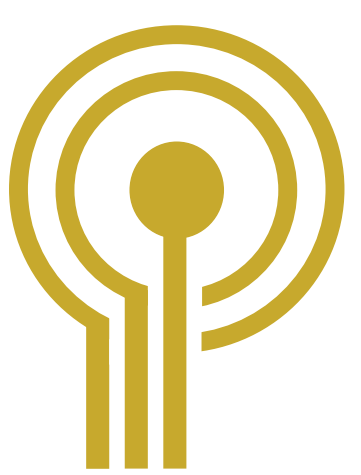

(b)

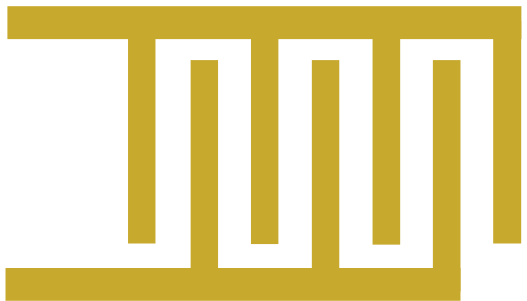

(c)

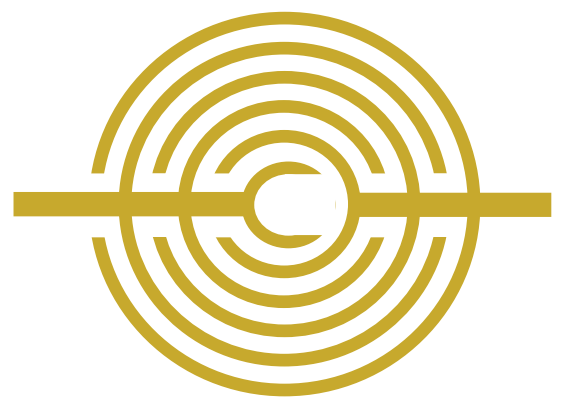

(d)

FIGURE 20: Configurations of electrodes: (a) linear 4-pole; (b) concentric; (c) interdigitated 2-pole, linear; (d) interdigitated 2-pole, concentric. 


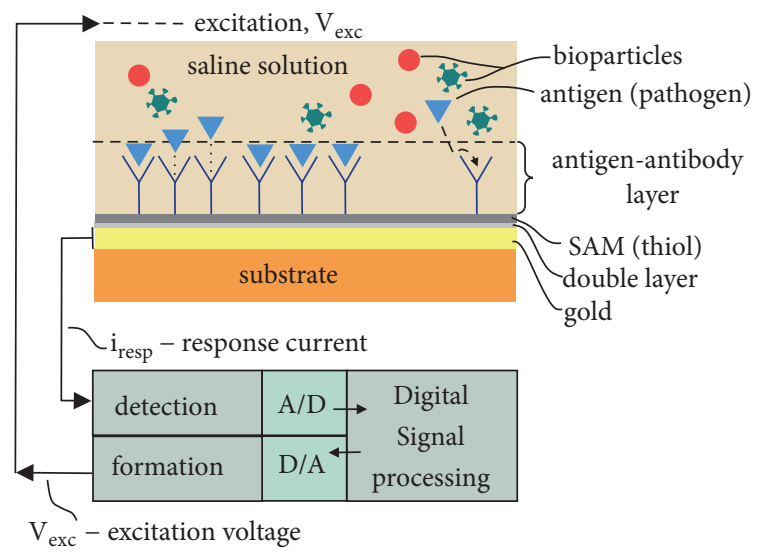

FIGURE 21: Affinity biosensor for bioimpedance detection of pathogens in biological solution.

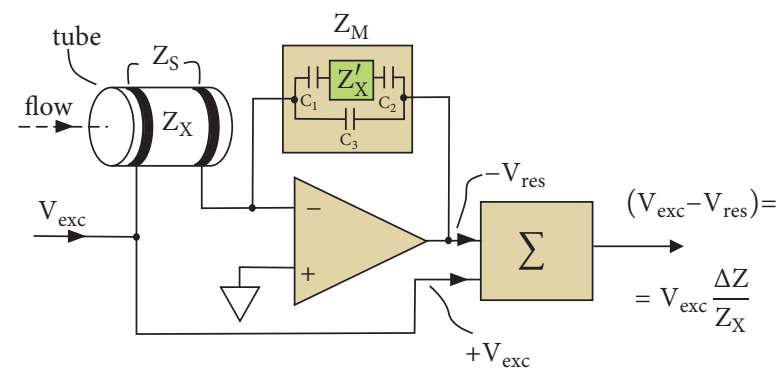

FIGURE 22: Biosensor with insulated electrodes on the tube form channel.

$\Delta \mathrm{R}_{1}$ is caused by binding of antigens to antibodies (formation of the antigen-antibody layer). Presence of capacitance $\mathrm{C}_{1}$ brings us the reason of arising capacitive current through SAM and antigen-antibody layer, also between the bioparticles in the saline solution. This current is limited by the resistor $\mathrm{R}_{2}$ at higher frequencies, but overall capacitive current continues flowing through the parasitic stray capacitance $C_{p}$. Despite the fact that only changing resistive component, $\Delta \mathrm{R}_{1}$, is expected to be an informative factor, this change reflects in both, in real and imaginary parts $\operatorname{Re}\left[Z_{x}(j \omega)\right]$ and $\operatorname{Im}\left[Z_{x}(j \omega)\right]$ of the complex impedance $Z_{x}(j \omega)$. Extraction of $\Delta R_{1}$ requires spectral measurements at least over the range of medium frequencies.

In Figure 22, there is given a case of measurement with electrodes fully insulated from biological material. A glass microtube (100 $\mu \mathrm{m}$ inner diameter, $50 \mu \mathrm{m}$ thick wall) is used for the measurement of impedance of biosolution flowing through [344]. Because of absence of contact between ionic solution and surrounding metallic ring electrodes, also the double layer is absent. Instead of, nearly ideal capacitances $\mathrm{C}_{1}$ and $\mathrm{C}_{2}$ exist between the electrodes and solution, which is characterized now by $n=1$ in (27) and in Figure 19, $C_{e}(j \omega)$ transforms to the impedance of capacitor $Z_{c}(j \omega)=1 /(j \omega C)$.

The measurement configuration in Figure 22 is based on the structure of inverting operational amplifier with electrical model (impedance $Z_{M}$ ) in the feedback circuit and physical sensors with impedance $Z_{S}$ at the input. Complex impedance

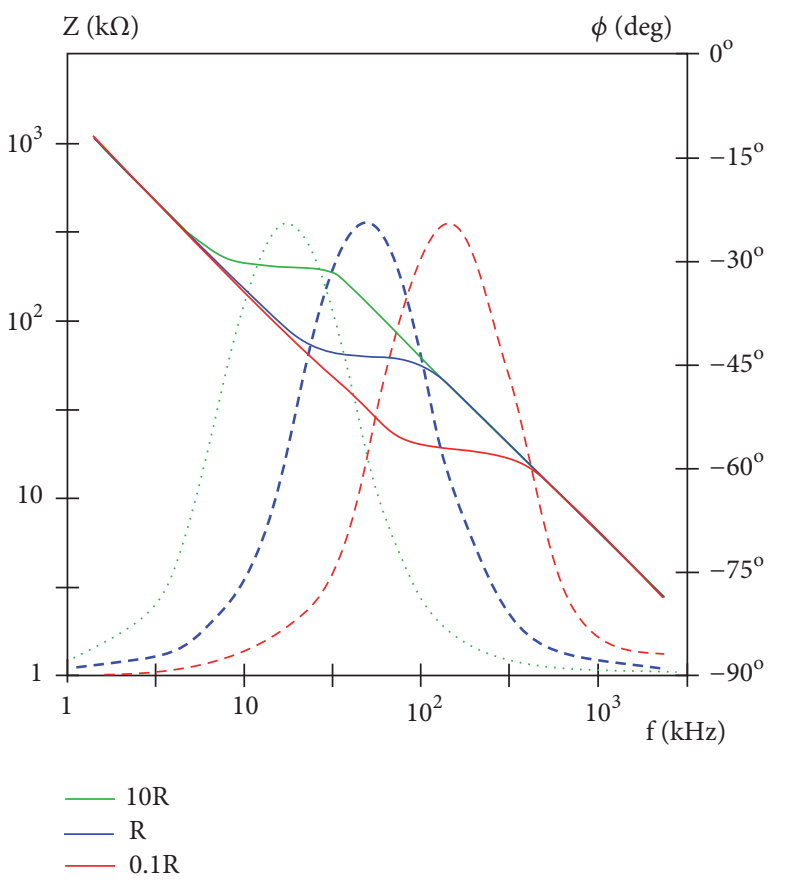

FIGURE 23: Frequency dependence of magnitude $Z_{S}(\omega)$ and phase $\varphi_{S}(\omega)$ of sensor impedance $Z_{S}(j \omega)$ at different values of concentration expressed through the resistive impedance $\mathrm{Z}$ in the graph. Informative value is measured at such frequencies, at which the phase curves obtain their maximal value about $-25^{\circ}$.

of the model $Z_{M}(j \omega)$ and of the real sensor $Z_{S}(j \omega)$ must be as similar as possible in the wide range of frequency $\omega=2 \pi \mathrm{f}$. As a result, the output signal of electronic summation $\Sigma$ is proportional to the relative change $\Delta \mathrm{Z}_{\mathrm{x}} / \mathrm{Z}_{\mathrm{x}}$ of the impedance to be measured; in more detail, please read [344].

How to extract the value of resistance $\mathrm{R}_{1}$, and its changing component $\Delta \mathrm{R}_{1}$, which carries primary information? The most reasonable way is to measure the impedance at the frequency, at which the phase shift $\varphi$ obtains its minimal value (Figure 23). At this frequency the impedance turns to its real value. For finding that frequency we have to measure in some range of frequencies, that is, to put through impedance spectroscopy around the expected frequency.

An experimental device for impedance-based counter of cells (cytometer) together with impedance-based characterization and sorting of cells and/or droplets is given in Figure 24 [348]. This device uses 4-electrode system having two pairs of electrodes, one pair for current excitation $\mathrm{i}_{\text {exc }}$ outside the channel, and the other one for pick-up the voltage response $\mathrm{V}_{\text {res }}$ inside the channel. Spectral analysis of cell impedance enables to differentiate the cells and count them separately using automatic decision maker operating on the bases of measurement results.

In Figures 25 and 26 there are described two practical versions of differential impedance measurement without using of some electronic reference circuits as designed for the device in Figure 22; see impedance $Z_{M}$ [344]. The both are for detecting and comparative analyzing of cells, i.e., the both are specific cytometers. In the first version 


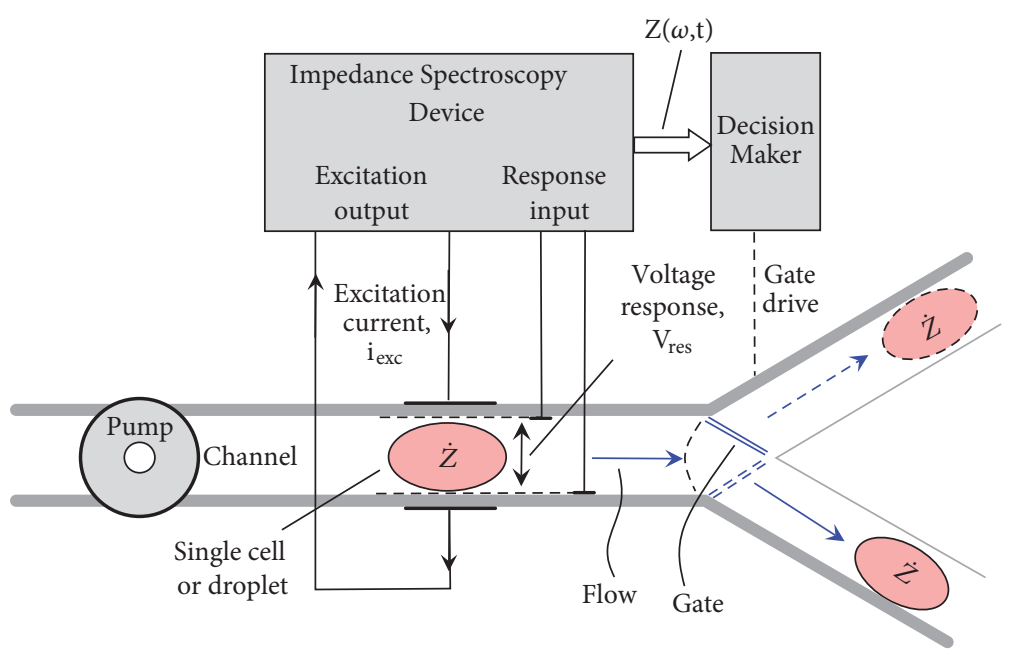

FIGURE 24: A cell or droplet sorter based on time dependent impedance spectroscopy (adapted from [348]).

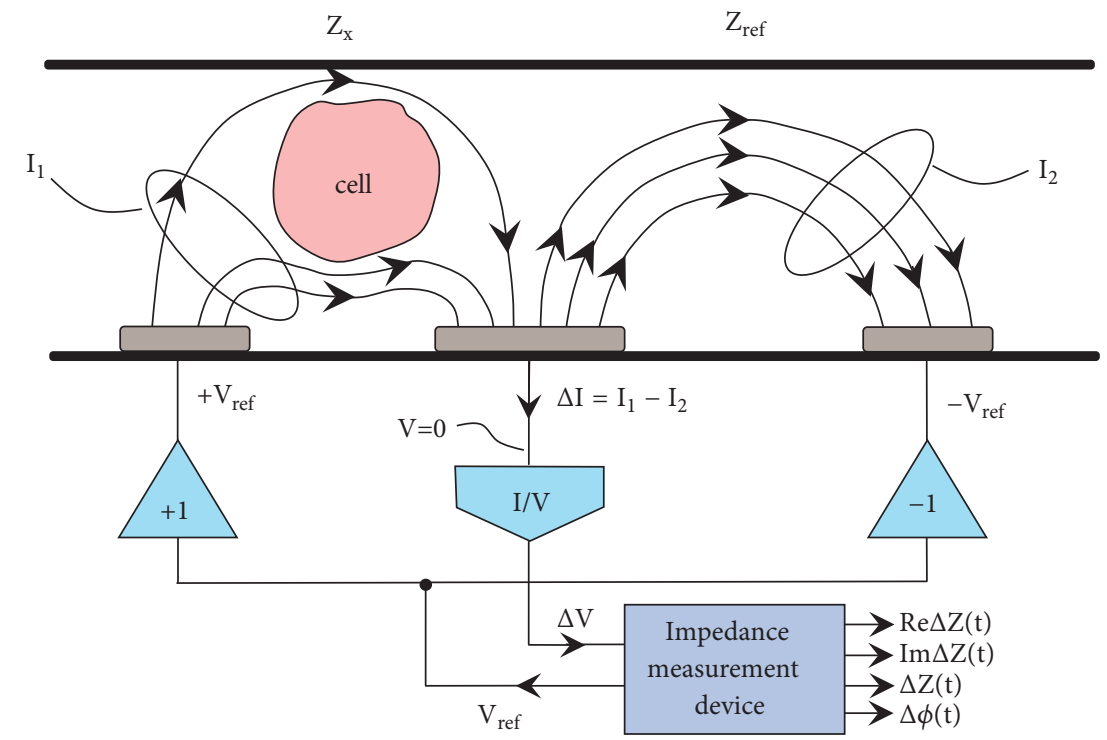

FIGURE 25: Three electrode system for the differential cell counting and characterizing.

(Figure 25), the opposite phase excitation signal as references $+\mathrm{V}_{\text {ref }}$ and $-\mathrm{V}_{\text {ref }}$ are generated. If the reference impedance $Z_{x}$ and impedance $Z_{\text {ref }}$ are equal (no cells in the channel), then the response current $\Delta \mathrm{I}$ is absent. But if the cell is present in channel, then $\mathrm{Z}_{\mathrm{x}}$ becomes higher than $\mathrm{V}_{\text {ref }}$ at lower frequencies (cell membrane does not conduct low-frequency currents). This phenomenon is the bases for the detecting and characterization of cells.

Similar principle is in use in Figure 26, where only a single excitation voltage $V_{\text {exc }}$ is applied to the both impedance $Z_{x}$ and impedance $Z_{\text {ref. }}$. Separate output currents, $I_{\text {res }}$ and $I_{\text {ref }}$, which will be subtracted from each other for yielding the difference $\Delta \mathrm{I}$. Significant difference between these currents $(\Delta \mathrm{I}>0)$ produces the voltage difference $\Delta \mathrm{V}$ at the output of current-to-voltage converter $\mathrm{I} / \mathrm{V}$. The voltage difference $\Delta \mathrm{V}$ signal indicates the presence of a cell in the channel.

\section{Future Challenges of Bioimpedance}

Despite all the advances made in bioimpedance for healthcare applications, there are still many challenges and open study fields for future research.

The development of wearable technologies has had a spectacular growth in recent years (heart rate, body temperature, physical activity, etc.). Important research efforts are carried out so that the information provided by these biomedical sensors can be really useful (remote monitoring of patients, early detection of anomalies, promotion of selfcare, etc.) towards an improvement in health and quality of life. There has been a paradigm shift in healthcare, moving from a curative medicine to a personalized, preventive, and proactive medicine $[134,349]$. This evolution is supported by the development of wearable biomedical devices for the management of personal health, which allow users to monitor 


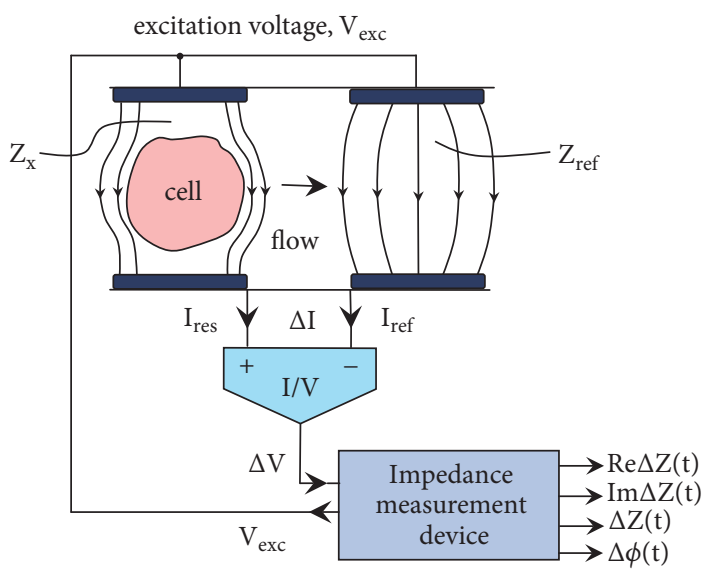

FIGURE 26: Four-electrode system for the differential cell counting and characterizing.

their physiological parameters continuously throughout the day. This functionality increases people's awareness of their current state of health and allows them to make adjustments in their lifestyle or seek early medical attention when the prognosis of the disease may be more favorable [134, 349]. As mentioned in previous sections, bioimpedance devices can provide multiple physiological parameters, and a huge interest is represented by the continuous monitoring of them. This circumstance poses the challenge of portability in bioimpedance systems, since the typical applications of bioimpedance measurement on the human body (BIA, ICG, TIP, EIT, and EDA) use electrodes on the skin. An obstacle for the development of portable bioimpedance devices is that a suitable electrode system is yet an open issue for wearable applications. These electrodes should be comfortable, easy to place by the user and should provide reliable measurements [95]. Aspects such as the material, size, shape, and disposition of the electrodes are a still open study area in contact electrodes for a nonobstructive use. As the contact electrodes like $\mathrm{Ag} / \mathrm{AgCl}$ are not suitable for continuous use, new methods based on conductive textiles and other noncontact coupling methods are being investigated. The use of noncontact electrodes can solve this problem, but it raises others as a greater sensitivity to the artifacts of movement and the electrical conditions of the environment and the human body [350]. Although the specific placement of the electrodes for BIA, ICG, EIT, and TIP applications has been widely discussed in the literature, there is a lack of information on the performances and issues about their application in a compact configuration more suitable for a portable environment, such as a patch or a textile garment [351]. The understanding of the current and voltage distribution mechanisms inside the body will be essential to optimize the sensitivity of bioimpedance devices in portable applications [351].

The standard methods of bioimpedance usually require the interaction of the doctor/nurse with the patient (placement of cables, electrodes, device management, etc.), so that these measurement systems are only adequate in a clinical setting. However, in an ideal healthcare application, the patient must forget that he/she is being monitored, for a realistic evaluation under conditions of daily life. Bioimpedance devices must be seamless to the user and carers. To that aim, important challenges are related to the miniaturization and contact-free and unobtrusive use of bioimpedance systems, which must also have processing and wireless communication capabilities for its integration into an e-Health system. The bioimpedance acts as an additional source of data, with the ability to integrate information into the electronic medical record and the consequent interoperability needs. It is necessary to establish a trade-off between the comfort of the user, the capacity for long-term use and the wireless communications of reliable data. The complexity of the bioimpedance systems is usually quite high (current injection, voltage measurement, demodulation, processing, etc.) [351] and the use of high frequency signals (from tens to hundreds of $\mathrm{kHz}$ ) generally requires a high energy consumption [351], so this new horizon involves a hardware optimization in size, robustness, and precision, but also in energy consumption, to maximize the autonomy of the devices, fundamental in the case of implants $[116,352]$. A key point of bioimpedance systems is the improvement of the current source. A research effort must be made to increase the bandwidth and the output impedance of the current source to improve the accuracy of the measurements and make the devices robust against load bioimpedance variations [112]. As the digital signal processing and wireless communications are the main sources of energy consumption in sensor devices, any progress in both the hardware and the processing algorithms will have a positive impact on increasing energy efficiency $[140,353]$. An alternative may be the use of comparators and timers instead of ADC to reduce the energy consumption associated with processing [354].

The improvement of sensitivity is highlighted as a study objective in bioimpedance equipment development, since the biological process to be monitored can lead to very small signal variations [355]. An adaptive preconditioning can be convenient for this purpose. The application of new approaches like nanotechnologies can improve the sensitivity of bioimpedance systems, as already it has been demonstrated in the case of the incorporation of carbon nanofibers on the electrodes of electrochemical sensors [96] or graphene electrodes for body measurements [267]. Due to the characteristics of an EIT system, with multiple electrodes and the need for processing algorithms, portability and real-time monitoring is an open challenge in the case of EIT.

Since the bioimpedance measurements are affected by multiple factors at the same time and it is very complicated to isolate the influence of the parameter to be measured, another challenge is the investigation of new models, equations, and methods to improve the specificity [356]. The problem of specificity is common to all bioimpedance methods. In BIA applications, the dependence of SFBIA method on a population regression study implies a loss of specificity in situations different from that of the study. Although BIS method is more robust in this sense, more extensive investigations must still be carried out. In bioimpedance measurements of biological samples, the concentration of the metabolite or substance to be detected can be masked by the presence of another element that affects electrically in the same way. An EIT image is 
naturally fuzzy because the electric currents diffuse inside a volume. However, new EIT models, measurement schemes and electrode configurations can provide a higher spatial resolution. The application of new processing algorithms, more complex and taking into account a greater number of factors, can also allow an improvement in specificity.

As mentioned in Section 2.6, multiple sources of noise and artifacts can affect bioimpedance measurements. High frequency measurement devices suffer from significant distortions in the phase [357]. Research that minimizes these effects must be done at the hardware level, but also at the processing level to detect and correct its effects. The phenomena that introduce errors in bioimpedance measurement should be investigated in greater depth to incorporate these parameters in the processing algorithms [358]. In addition, the context of continuous monitoring imposes an even greater challenge, since artifacts due to movement and parasitic capacitances can be even greater. To reduce errors, bioimpedance systems use calibrations; however, there is no standard calibration protocol for the different bioimpedance applications. Standardized protocols should also be established when carrying out studies with bioimpedance, to allow evaluating the results of different authors on the same conditions and with the electrodes in the same positions.

The anisotropic properties of the skin and tissues must be taken into account, since the flow of the electric current inside the human body is modified by the composition and orientation of the cells in the tissues $[88,359]$. Since the tetrapolar configuration of electrodes, which is commonly used in bioimpedance measurements, is affected by the anisotropic properties of blood vessels, muscle and nerve fibers [98], new electrode configurations and methods must be investigated to mitigate their effects [38] or allow their measurement as a diagnostic parameter of diseases [98, 99, 360]. Moreover, the anisotropy is of special relevance in EIT and its incorporation to image reconstruction models using tensors of anisotropic conductivity can provide a higher resolution [361].

Advances in miniaturization of IC technologies, biocompatible materials and wireless communication technologies have made implants more and more feasible for clinical use. To the extent, bioimpedance technology can provide a characterization of a tissue, and taking into account the fact that it is safe, simple and of low-cost, this technology has been investigated for its incorporation in implants such as cardiac pacemakers for the objective evaluation of the cardiac and respiratory process [140], in stimulation intracranial electrodes or cochlear implants to assess the state of the implant [362], to help in the process of placement of an implant [363] or in the detection of prostate cancer with microendoscopic probes [37]. Future advances in the field of implants will give rise to new challenges in the application of bioimpedance, to serve as a complement of the implant, but also serve as a basis in innovative implantable devices for in situ monitoring of a physiological process.

Biosensors and Lab-on-Chip technologies based on bioimpedance are of great interest in biological, medical, and industrial fields, mainly due to their low-cost and speed in the measurement, and because they can be used in continuous monitoring processes. The development of novel implants could also benefit from these technologies. However, the problems of precision and sensitivity are a major challenge in this type of applications. In-depth investigations must be carried out to accurately model the behavior of the biological impedance of these systems. As previously discussed, specificity is a major problem that can be solved by antibodies or artificially synthesized aptamers, still in the process of development. Implementation of bioimpedance sensing in Lab-on-Chip based technology for point-of-care (PoC) and need-of-care (NoC) devices [364] opens new perspectives to prevent of epidemic spread of infectious diseases in developing countries, and environmental and social disasters, everywhere. New implantable sensors can be designed as well on the bases of bioimpedance sensing [365]. The biosensors can detect cell nutrients such as glucose, metabolites such as lactate, cell density and $\mathrm{pH}$ in biological processes, but the field of research is open to new parameters and substances. Besides, the sample preparation process imposes important portability restrictions in some applications, hindering the development of this technology.

In this work, a review of the most recent applications of bioimpedance has been carried out, but in all of them there are some challenges that must be addressed. Although the estimation of body composition by bioimpedance has multiple advantages (noninvasive, painless, safe, simple and nonionizing-radiation-based method [103]) and exhibits a strong correlation with reference methods [210], this technique has not yet been accepted in routine clinical practice, possibly due to artifacts, systematic errors and estimation errors that occur in the measurements [366]. The algorithms used in commercial devices are usually unknown and there is little information about their validation in a clinical setting [103]. Clinical studies in which these devices have been used are limited by the small sample size [151] and additional research is needed with larger and more heterogeneous population samples, especially in obese individuals, subjects with low weight and in population groups with unique anthropometric characteristics or in altered states of composition [210]. To improve the error, innovative and individualized approaches must be developed to improve the accuracy of the bioimpedance methods [193].

In a typical bioimpedance body estimation, the electrodes are usually placed on the hand and the foot due to the multiple benefits that this configuration provides (reduction of the variability of the position, simply and easy measurement, the user does not need to undress, possibility of patient evaluation in bed, etc.) [210]. However, this location may not be optimal for assessing the specific composition in a corporal segment, especially in those patients who suffer extreme changes in body composition, such as patients on hemodialysis or patients with spinal cord injury [367]. The measurement of segmental and localized bioimpedance is an area of study that requires additional research [210, 367].

Despite the maturity of ICG, there are still doubts about whether this technique can really reflect the cardiac function and the filling pressures in the heart [368], especially under unnormal health conditions. The data provided in most of the studies are small and often are not controlled. More extensive 
studies must be conducted to test the clinical utility of this technology and extend its application beyond the scope of the research. A disadvantage of ICG is that the distances between the measuring electrodes are high, and thus inaccuracies may arise in the estimation of parameters due to the own cardiorespiratory dynamics [369]. New methods based on ensemble averaging, wavelet transform, or signal filtering can address this inaccuracy $[369,370]$.

Despite the low resolution of the EIT technology for its application in medical images, which results in a smoothing effect, this technology stands out because functional information related to the internal body bioimpedance compartments is generated. In this way, EIT provides added value when it is applied in combination with other diagnostic methods. EIT is a very active study area as represents a low-cost and harmless method that can be used to obtain images of organs, tumors and blood vessels, although with low spatial resolution [371]. This method is very sensitive to modeling and measurement errors, and a small difference can lead to a large error in the reconstruction [372]. Advances in more accurate body models and in technology to provide increased stability and SNR will lead to an improvement in image resolution $[26,373]$. Many researchers use phantoms that mimic body tissues to calibrate and test EIT systems [374]. As the intracellular compartment can only be observed at high frequencies, EIT usually needs a high bandwidth that is affected by the parasitic capacitance of the cables and switches. One way to solve the inherent inaccuracy of this technology can be the fusion of different sources of information, but also the parallel use of diverse technologies. Some research groups are investigating new schemes to improve the bandwidth by using multiple current injectors and voltmeters in parallel [372]. Other approaches employ active electrodes to mitigate the effects of parasitic capacitances $[375,376]$. The collaboration of technical experts, mathematicians, and clinicians will be necessary to make a trade-off between the hardware and software complexity and the precision performance.

Bioimpedance based sensing methods have found an established usage in some types of biosensors (e.g., for single cell counting and sorting), but their implementation in biotechnology and medicine is still a topic of future developments. Excellent perspectives are opening in detection of pathogens for preventing epidemic spread of infectious diseases in developing countries, and environmental and social disasters, everywhere. Implementation of bioimpedance sensing in Lab-on-Chip based technology for point-of-care $(\mathrm{PoC})$ and need-of-care $(\mathrm{NoC})$ devices [364] opens new perspectives for prevention of the abovementioned threats.

Although bioimpedance measurement technology is a mature method, a major research effort must be made in conjunction with experts in clinical diagnosis to improve the sensitivity, the specificity, and the confidence in the use of technology in routine clinical practice. Another challenge faced by bioimpedance is the research and development of new applications, in which the detection of cancer will have a prominent position $[31,37,65,68]$, but other new applications are feasible such as lymphoedema evaluation
[43, 48], vital sign monitoring in automotive environments [377], noninvasive cuffless blood pressure estimation [378381], electrical impedance myography [382], glucose concentration assessment $[383,384]$, noninvasive detection of physiological processes [385], diagnosis of diseases of peripheral vascular system $[43,386]$, evaluation of elbow joint motion [387], detection of bacteria populations in water solutions [388], evaluation of the status of wound healing [389], infiltration detection during intravenous therapy [390], blood flow assessment [43], and characterization of an implantable neurostimulator [391], as a surgical aid tool [101, 392] or in the evaluation of transplanted organs [46].

\section{Acronyms}

ADC: Analog-to-digital converter

AFE: Analog-front-end

ASIC: Application-specific integrated circuit

BIA: Bioelectrical impedance analysis

BIS: Bioimpedance spectroscopy

BIVA: Bioelectrical impedance vector analysis

BMI: Body mass index

BrD: $\quad$ Bromide dilution

CI: $\quad$ Cardiac index

CMOS: Complementary metal-oxide-semiconductor

CMRR: Common-mode rejection ratio

CO: Cardiac output

CoIn: Confidence interval

CPE: Constant phase element

$\mathrm{D}_{2} \mathrm{O}:$ Deuterium oxide dilution

DAC: Digital-to-analog converter

DEXA: Dual-energy X-ray absorptiometry

ECG: Electrocardiogram

ECW: Extracellular water

EDA: Electrodermal activity

EIS: $\quad$ Electrochemical impedance spectroscopy

EIT: Electrical impedance tomography

FFM: Fat free mass

FE: $\quad$ Finite-element

FFT: Fast Fourier Transform

FM: $\quad$ Fat mass

FPGA: Field-programmable gate array

IC: Integrated circuit

ICG: Impedance cardiography

ICW: Intracellular water

LoC: Lab-on-a-chip

LVET: Left-ventricular ejection time

MFBIA: Multifrequency Bioimpedance Analysis

MRI: Magnetic resonance imaging

NLLS: Nonlinear least squares

NoC: Need-of-care

OPAM: Operational amplifier

PEEP: Positive end-expiratory pressure

PET: Positron-emission tomography

PoC: Point-of-care

PPG: Photoplethysmography

PWV: Pulse wave velocity

SAM: Self-assembling monolayer

SCR: Skin conductance response 
SFBIA: Single frequency bioimpedance analysis

SPECT: Single-photon emission computed tomography

SNR: Signal-to-noise ratio

SV: $\quad$ Stroke volume

TBK: Total body potassium

TBW: Total body water

TIP: Transthoracic impedance pneumography

TrD: Tritium dilution

VCCS: Voltage-controlled current source.

\section{Conflicts of Interest}

The authors declare that there are no conflicts of interest with any company or organization regarding the material discussed in this paper.

\section{Acknowledgments}

This work was supported in part by the "Fondo de Investigaciones Sanitarias" (Instituto de Salud Carlos III, Spain) under Grants PI15/00306 and DTS15/00195, in part by the "Fundación Progreso y Salud" (Government of Andalucía, Spain) under Grants PI-0010-2013, PI-0041-2014, and PIN0394-2017, in part by "Fundación Mutua Madrileña" under grant VÍA-RENAL, in part by Estonian Research Council (Grant IUT1911) and European Regional Development Fund in frames of Estonian ICT Center EXCITE, and in part by the projects H2020-2014-2-668995-Cognitive Electronics and H2020-FLAG-ERA-JTC2016-CONVERGENCE.

\section{References}

[1] E. Borelli, G. Paolini, F. Antoniazzi et al., "Habitat: an IoT solution for independent elderly," Sensors, vol. 19, no. 5, 2019.

[2] S. Majumder, T. Mondal, and M. J. Deen, "A simple, low-cost and efficient gait analyzer for wearable healthcare applications," IEEE Sensors Journal, vol. 19, no. 6, pp. 2320-2329, 2019.

[3] R. Punj and R. Kumar, "Technological aspects of WBANs for health monitoring: a comprehensive review," Wireless Networks, vol. 25, no. 3, pp. 1125-1157, 2019.

[4] F. Riaz, M. A. Azad, J. Arshad, M. Imran, A. Hassan, and S. Rehman, "Pervasive blood pressure monitoring using Photoplethysmogram (PPG) sensor," Future Generation Computer Systems, vol. 98, pp. 120-130, 2019.

[5] L. Haoyu, L. Jianxing, N. Arunkumar, A. F. Hussein, and M. M. Jaber, "An IoMT cloud-based real time sleep apnea detection scheme by using the $\mathrm{SpO} 2$ estimation supported by heart rate variability," Future Generation Computer Systems, vol. 98, pp. 69-77, 2019.

[6] M. S. Mahmud, H. Fang, and H. Wang, "An integrated wearable sensor for unobtrusive continuous measurement of autonomic nervous system," IEEE Internet of Things Journal, vol. 6, no. 1, pp. 1104-1113, 2019.

[7] Y. Li, S. Li, H. Song, B. Shao, X. Yang, and N. Deng, "Noninvasive blood pressure estimation with peak delay of different pulse waves," International Journal of Distributed Sensor Networks, vol. 15, no. 3, 2019.
[8] K. Xu, Y. Lu, and K. Takei, "Multifunctional skin-inspired flexible sensor systems for wearable electronics," Advanced Materials Technologies, vol. 4, no. 3, 2019.

[9] J. Harvey, S. Salehizadeh, Y. Mendelson, and K. Chon, "OxiMA: a frequency-domain approach to address motion artifacts in photoplethysmograms for improved estimation of arterial oxygen saturation and pulse rates," IEEE Transactions on Biomedical Engineering, vol. 66, no. 2, pp. 311-318, 2019.

[10] M. Rapin, F. Braun, A. Adler et al., "Wearable sensors for frequency-multiplexed EIT and multilead ECG data acquisition," IEEE Transactions on Biomedical Engineering, vol. 66, no. 3, pp. 810-820, 2019.

[11] E. Piuzzi, S. Pisa, E. Pittella, L. Podesta, and S. Sangiovanni, "Low-cost and portable impedance plethysmography system for the simultaneous detection of respiratory and heart activities," IEEE Sensors Journal, vol. 19, no. 7, pp. 2735-2746, 2019.

[12] L. M. Roa, D. Naranjo, J. Reina-Tosina et al., "Applications of bioimpedance to end stage renal disease (ESRD)," Studies in Computational Intelligence, vol. 404, pp. 689-769, 2013.

[13] S. Kugananthan, Z. Gridneva, C. T. Lai et al., "Associations between maternal body composition and appetite hormones and macronutrients in human milk," Nutrients, vol. 9, no. 3, 2017.

[14] L. Mullie, A. Obrand, M. Bendayan et al., "Phase angle as a biomarker for frailty and postoperative mortality: the BICS study," Journal of the American Heart Association, vol. 7, no. 17, Article ID e008721, 2018.

[15] M. S. Mundi, J. J. Patel, and R. Martindale, "Body composition technology: implications for the ICU," Nutrition in Clinical Practice, vol. 34, no. 1, pp. 48-58, 2019.

[16] E. M. Widen, I. Tsai, S. M. Collins et al., "HIV infection and increased food insecurity are associated with adverse body composition changes among pregnant and lactating Kenyan women," European Journal of Clinical Nutrition, vol. 73, no. 3, pp. 474-482, 2019.

[17] A. Ruiz-Vargas, J. W. Arkwright, and A. Ivorra, "A portable bioimpedance measurement system based on red pitaya for monitoring and detecting abnormalities in the gastrointestinal tract," in Proceedings of the 2016 IEEE-EMBS Conference on Biomedical Engineering and Sciences, IECBES 2016, pp. 150-154, December 2016.

[18] M. V. Fedewa, B. S. Nickerson, and M. R. Esco, "Associations of body adiposity index, waist circumference, and body mass index in young adults," Clinical Nutrition, vol. 38, no. 2, pp. 715720, 2019.

[19] S. Lai, M. Muscaritoli, P. Andreozzi et al., "Sarcopenia and cardiovascular risk indices in patients with chronic kidney disease on conservative and replacement therapy," Nutrition Journal, vol. 62, pp. 108-114, 2019.

[20] X. Zhang, J. Zheng, L. Zhang et al., "Systemic inflammation mediates the detrimental effects of obesity on asthma control," Allergy and Asthma Proceedings, vol. 39, no. 1, pp. 43-50, 2018.

[21] T. R. Ackland, T. G. Lohman, J. Sundgot-Borgen et al., "Current status of body composition assessment in sport: review and position statement on behalf of the Ad Hoc research working group on body composition health and performance, under the auspices of the I.O.C. medical commission," Sports Medicine, vol. 42, no. 3, pp. 227-249, 2012.

[22] A. Hafid, S. Benouar, M. Kedir-Talha, F. Abtahi, M. Attari, and F. Seoane, "Full impedance cardiography measurement device using raspberry PI3 and system-on-chip biomedical instrumentation solutions," IEEE Journal of Biomedical and Health Informatics, vol. 22, no. 6, pp. 1883-1894, 2018. 
[23] S. Said and G. T. Hernandez, "The link between chronic kidney disease and cardiovascular disease," Journal of Nephropathology, vol. 3, no. 3, pp. 99-104, 2014.

[24] W. Lee and S. Cho, "Integrated all electrical pulse wave velocity and respiration sensors using bio-impedance," IEEE Journal of Solid-State Circuits, vol. 50, no. 3, pp. 776-785, 2015.

[25] W. Lee and S. H. Cho, "An integrated pulse wave velocity sensor using Bio-impedance and noise-shaped body channel communication," in Proceedings of the 2013 Symposium on VLSI Circuits, VLSIC 2013, pp. C218-C219, June 2013.

[26] M. Fernández-Corazza, S. Turovets, P. Luu, N. Price, C. H. Muravchik, and D. Tucker, "Skull modeling effects in conductivity estimates using parametric electrical impedance tomography," IEEE Transactions on Biomedical Engineering, vol. 65, no. 8, pp. 1785-1797, 2018.

[27] G. Boverman, D. Isaacson, J. C. Newell et al., "Efficient simultaneous reconstruction of time-varying images and electrode contact impedances in electrical impedance tomography," IEEE Transactions on Biomedical Engineering, vol. 64, no. 4, pp. 795806, 2017.

[28] Z. Zhao, M. Chang, M. Chang et al., "Positive end-expiratory pressure titration with electrical impedance tomography and pressure-volume curve in severe acute respiratory distress syndrome," Annals of Intensive Care, vol. 9, no. 1, 2019.

[29] B. Sun, S. Yue, Z. Hao, Z. Cui, and H. Wang, "An improved tikhonov regularization method for lung cancer monitoring using electrical impedance tomography," IEEE Sensors Journal, vol. 19, no. 8, pp. 3049-3057, 2019.

[30] M. Takhti and K. Odame, "Structured design methodology to achieve a high SNR electrical impedance tomography," IEEE Transactions on Biomedical Circuits and Systems, vol. 13, no. 2, pp. 364-375, 2019.

[31] P. Kassanos, L. Constantinou, I. F. Triantis, and A. Demosthenous, "An integrated analog readout for multi-frequency bioimpedance measurements," IEEE Sensors Journal, vol. 14, no. 8, pp. 2792-2800, 2014.

[32] R. E. Fernandez, E. Lebiga, A. Koklu, A. C. Sabuncu, and A. Beskok, "Flexible bioimpedance sensor for label-free detection of cell viability and biomass," IEEE Transactions on NanoBioscience, vol. 14, no. 7, pp. 700-706, 2015.

[33] G. Huertas, A. Maldonado, A. Yufera, A. Rueda, and J. L. Huertas, "The bio-oscillator: a circuit for cell-culture assays," IEEE Transactions on Circuits and Systems II: Express Briefs, vol. 62, no. 2, pp. 164-168, 2015.

[34] F. A. Alexander, D. T. Price, and S. Bhansali, "From cellular cultures to cellular spheroids: is impedance spectroscopy a viable tool for monitoring multicellular spheroid (MCS) drug models?" IEEE Reviews in Biomedical Engineering, vol. 6, pp. 63-76, 2013.

[35] R. Kraya, A. Komin, and P. Searson, "On chip bioelectric impedance spectroscopy reveals the effect of P-glycoprotein efflux pumps on the paracellular impedance of tight junctions at the blood-brain barrier," IEEE Transactions on NanoBioscience, vol. 15, no. 7, pp. 697-703, 2016.

[36] K. Yu, Q. Shao, S. Ashkenazi, J. C. Bischof, and B. He, "In vivo electrical conductivity contrast imaging in a mouse model of cancer using high-frequency magnetoacoustic tomography with magnetic induction (hfMAT-MI)," IEEE Transactions on Medical Imaging, vol. 35, no. 10, pp. 2301-2311, 2016.

[37] S. Khan, A. Mahara, E. S. Hyams, A. R. Schned, and R. J. Halter, "Prostate cancer detection using composite impedance metric,"
IEEE Transactions on Medical Imaging, vol. 35, no. 12, pp. 25132523, 2016

[38] K. Dudzinski, M. Dawgul, K. D. Pluta, B. Wawro, W. Torbicz, and D. G. Pijanowska, "Spiral concentric two electrode sensor fabricated by direct writing for skin impedance measurements," IEEE Sensors Journal, vol. 17, no. 16, pp. 5306-5314, 2017.

[39] S. Ahmad, I. Batkin, O. Kelly, H. R. Dajani, M. Bolic, and V. Groza, "Multiparameter physiological analysis in obstructive sleep apnea simulated with mueller maneuver," IEEE Transactions on Instrumentation and Measurement, vol. 62, no. 10, pp. 2751-2762, 2013.

[40] P. Walsh, O. Escalona, J. Velasquez, N. Waterman, and P. Rodrigues, "Impedance compensated passive implantable atrial defibrillator," IEEE Electronics Letters, vol. 50, no. 17, pp. 11921193, 2014.

[41] L. Sornmo, F. Sandberg, E. Gil, and K. Solem, "Noninvasive techniques for prevention of intradialytic hypotension," IEEE Reviews in Biomedical Engineering, vol. 5, pp. 45-59, 2012.

[42] C. Brendle, B. Rein, A. Niesche et al., "Electrical bioimpedancecontrolled surgical instrumentation," IEEE Transactions on Biomedical Circuits and Systems, vol. 9, no. 5, pp. 743-750, 2015.

[43] S. Hersek, H. Toreyin, and O. T. Inan, "A robust system for longitudinal knee joint edema and blood flow assessment based on vector bioimpedance measurements," IEEE Transactions on Biomedical Circuits and Systems, vol. 10, no. 3, pp. 545-555, 2016.

[44] S. Hersek, H. Toreyin, C. N. Teague et al., "Wearable vector electrical bioimpedance system to assess knee joint health," IEEE Transactions on Biomedical Engineering, vol. 64, no. 10, pp. 2353-2360, 2017.

[45] C. J. Chester, P. T. Gaynor, R. D. Jones, and M. Huckabee, "Electrical bioimpedance measurement as a tool for dysphagia visualisation," Healthcare Technology Letters, vol. 1, no. 3, pp. 115-118, 2014.

[46] O. I. Al-Surkhi and R. Y. Naser, "Detection of cell morphological changes of ischemic rabbit liver tissue using bioimpedance spectroscopy," IEEE Transactions on NanoBioscience, vol. 17, no. 4, pp. 402-408, 2018.

[47] H. Wang, Y. He, Q. Yan et al., "Correlation between the dielectric properties and biological activities of human ex vivo hepatic tissue," Physics in Medicine and Biology, vol. 60, no. 6, pp. 26032617, 2015.

[48] M. Asklöf, P. Kjølhede, N. B. Wodlin, and L. Nilsson, "Bioelectrical impedance analysis; a new method to evaluate lymphoedema, fluid status, and tissue damage after gynaecological surgery - A systematic review," European Journal of Obstetrics \& Gynecology and Reproductive Biology, vol. 228, pp. 111-119, 2018.

[49] C. Hawthorne, M. Shaw, I. Piper, L. Moss, and J. Kinsella, "Transcranial bioimpedance measurement as a non-invasive estimate of intracranial pressure," Acta Neurochirurgica, Supplementum, vol. 126, pp. 89-92, 2018.

[50] P. Langer, P. Jurák, V. Vondra et al., "Respiratory-induced hemodynamic changes measured by whole-body multichannel impedance plethysmography," Physiological Research, vol. 67, no. 4, pp. 571-581, 2018.

[51] M.-H. Jun, S. Kim, B. Ku et al., "Glucose-independent segmental phase angles from multi-frequency bioimpedance analysis to discriminate diabetes mellitus," Scientific Reports, vol. 8, no. 1, 2018.

[52] A. C. Buchholz, C. Bartok, and D. A. Schoeller, "The validity of bioelectrical impedance models in clinical populations," Nutrition in Clinical Practice, vol. 19, no. 5, pp. 433-446, 2004. 
[53] J. Castizo-Olier, A. Irurtia, M. Jemni, M. Carrasco-Marginet, R. Fernández-García, and F. A. Rodríguez, "Bioelectrical impedance vector analysis (BIVA) in sport and exercise: Systematic review and future perspectives," PLoS ONE, vol. 13, no. 6, 2018.

[54] S. Grimnes and Ø. Martinsen, Bioimpedance and Bioelectricity Basics, Academic Press, London, UK, 3rd edition, 2014.

[55] V. S. Teixeira, W. Krautschneider, and J. J. Montero-Rodriguez, "Bioimpedance spectroscopy for characterization of healthy and cancerous tissues," in Proceedings of the 2018 IEEE International Conference on Electrical Engineering and Photonics (EExPolytech), pp. 147-151, October 2018.

[56] D. Križaj, "Basics of numerical simulations of bioimpedance phenomena," in Bioimpedance in Biomedical Applications and Research, pp. 101-116, Springer International Publishing, 2018.

[57] C. Gabriel, S. Gabriel, and E. Corthout, "The dielectric properties of biological tissues: I. Literature survey," Physics in Medicine and Biology, vol. 41, no. 11, pp. 2231-2249, 1996.

[58] M. Lin, D. Hu, M. Marmor, S. Herfat, C. Bahney, and M. Maharbiz, "Smart bone plates can monitor fracture healing," Scientific Reports, vol. 9, no. 1, 2019.

[59] P. Bhardwaj, D. V. Rai, M. L. Garg, and B. P. Mohanty, "Potential of electrical impedance spectroscopy to differentiate between healthy and osteopenic bone," Clinical Biomechanics, vol. 57, pp. 81-88, 2018.

[60] M. Amini, J. Hisdal, and H. Kalvøy, "Applications of bioimpedance measurement techniques in tissue engineering," Journal of Electrical Bioimpedance (JEB), vol. 9, no. 1, pp. 142-158, 2018.

[61] S. Gabriel, R. W. Lau, and C. Gabriel, “The dielectric properties of biological tissues: III. Parametric models for the dielectric spectrum of tissues," Physics in Medicine and Biology, vol. 41, no. 11, pp. 2271-2293, 1996.

[62] M. D. O’Toole, L. A. Marsh, J. L. Davidson, Y. M. Tan, D. W. Armitage, and A. J. Peyton, "Non-contact multi-frequency magnetic induction spectroscopy system for industrial-scale bio-impedance measurement," Measurement Science and Technology, vol. 26, no. 3, 2015.

[63] A. De Lorenzo, A. Andreoli, J. Matthie, and P. Withers, "Predicting body cell mass with bioimpedance by using theoretical methods: a technological review," Journal of Applied Physiology, vol. 82, no. 5, pp. 1542-1558, 1997.

[64] A. Piccoli, L. D. Nescolarde, and J. Rosell, "Conventional and vectorial analysis of bioimpedance in clinical practice," Nefrología, vol. 22, no. 3, pp. 228-238, 2002.

[65] C. Carpano Maglioli, D. G. Caldwell, and L. S. Mattos, "A bioimpedance sensing system for in-vivo cancer tissue identification: Design and preliminary evaluation," in Proceedings of the 39th Annual International Conference of the IEEE Engineering in Medicine and Biology Society, EMBC 2017, pp. 4235-4238, July 2017.

[66] U. G. Kyle, I. Bosaeus, A. D. De Lorenzo et al., "Bioelectrical impedance analysis - part I: review of principles and methods," Clinical Nutrition, vol. 23, no. 5, pp. 1226-1243, 2004.

[67] T. J. Freeborn, "A survey of fractional-order circuit models for biology and biomedicine," IEEE Journal on Emerging and Selected Topics in Circuits and Systems, vol. 3, no. 3, pp. 416-424, 2013.

[68] J. Shell and W. D. Gregory, "Efficient cancer detection using multiple neural networks," IEEE Journal of Translational Engineering in Health and Medicine, vol. 5, pp. 1-7, 2017.
[69] H. Wu, Y. Zuo, F. Wei et al., "Study on complex Td correction and Cole-Cole parameters for the impedance of immunobiosensor," in Proceedings of the 2011 International Conference on New Technology of Agricultural Engineering, ICAE 2011, pp. 806-809, May 2011.

[70] R. Buendia, F. Seoane, I. Bosaeus et al., "Robustness study of the different immittance spectra and frequency ranges in bioimpedance spectroscopy analysis for assessment of total body composition," Physiological Measurement, vol. 35, no. 7, pp. 1373-1395, 2014.

[71] P. D. Jensen, P. M. Meaney, N. R. Epstien, and K. D. Paulsen, "Cole-cole parameter characterization of urea and potassium for improving dialysis treatment assessment," IEEE Antennas and Wireless Propagation Letters, vol. 11, pp. 1598-1601, 2012.

[72] M. Guermazi, O. Kanoun, and N. Derbel, "Investigation of long time beef and veal meat behavior by bioimpedance spectroscopy for meat monitoring," IEEE Sensors Journal, vol. 14, no. 10, pp. 3624-3630, 2014.

[73] K. Sasaki, K. Wake, and S. Watanabe, "A dosimetric study using best-fit Cole-Cole parameters of biological tissues and organs in radio frequency band," in Proceedings of the 2013 21st International Symposium on Electromagnetic Theory, EMTS 2013, pp. 630-633, May 2013.

[74] B. Rigaud, L. Hamzaoui, M. R. Frikha, N. Chauveau, and J.-P. Morucci, "In vitro tissue characterization and modelling using electrical impedance measurements in the $100 \mathrm{~Hz}-10 \mathrm{MHz}$ frequency range," Physiological Measurement, vol. 16, no. 3A, pp. A15-A28, 1995.

[75] M. Sezdi, M. Bayik, and Y. Ulgen, "Storage effects on the ColeCole parameters of erythrocyte suspensions," Physiological Measurement, vol. 27, no. 7, pp. 623-635, 2006.

[76] T. Dai and A. Adler, "In vivo blood characterization from bioimpedance spectroscopy of blood pooling," IEEE Transactions on Instrumentation and Measurement, vol. 58, no. 11, pp. 3831-3838, 2009.

[77] S. Kun, B. Ristic, R. Peura, and R. Dunn, "Algorithm for tissue ischemia estimation based on electrical impedance spectroscopy," IEEE Transactions on Biomedical Engineering, vol. 50, no. 12, pp. 1352-1359, 2003.

[78] O. I. Al-Surkhi, P. J. Riu, F. F. Vazquez, and J. Ibeas, "Monitoring Cole-Cole parameters during haemodialysis (HD)," in Proceedings of the 2007 29th Annual International Conference of the IEEE Engineering in Medicine and Biology Society, pp. 22382241, August 2007.

[79] C. Wang, M. Li, and M. Yao, "Impedance feature extraction of breast cancer and the surrounding tissues," in Proceedings of the 3rd International Conference on BioMedical Engineering and Informatics, BMEI 2010, vol. 3, pp. 922-926, October 2010.

[80] B. Maundy and A. S. Elwakil, "Extracting single dispersion Cole-Cole impedance model parameters using an integrator setup," Analog Integrated Circuits and Signal Processing, vol. 71, no. 1, pp. 107-110, 2012.

[81] I. Jesus and J. T. MacHado, "Application of Integer and Fractional Models in Electrochemical Systems," Mathematical Problems in Engineering, vol. 2012, Article ID 248175, 17 pages, 2012.

[82] A. De Lorenzo, N. Candeloro, A. Andreoli, and P. Deurenberg, "Determination of intracellular water by multifrequency bioelectrical impedance," Annals of Nutrition and Metabolism, vol. 39, no. 3, pp. 164-176, 1995. 
[83] A. H. Eldarrat, A. S. High, and G. M. Kale, "Age-related changes in ac-impedance spectroscopy studies of normal human dentine: further investigations," Journal of Materials Science: Materials in Medicine, vol. 21, no. 1, pp. 45-51, 2010.

[84] M. Tiitta and H. Olkkonen, "Electrical impedance spectroscopy device for measurement of moisture gradients in wood," Review of Scientific Instruments, vol. 73, no. 8, pp. 3093-3100, 2002.

[85] T. Repo, J. Laukkanen, and R. Silvennoinen, "Measurement of the tree root growth using electrical impedance spectroscopy," Silva Fennica, vol. 39, no. 2, pp. 159-166, 2005.

[86] R. L. Magin and M. Ovadia, "Modeling the cardiac tissue electrode interface using fractional calculus," Journal of Vibration and Control, vol. 14, no. 9-10, pp. 1431-1442, 2008.

[87] W. H. Huang, C. K. Chui, S. H. Teoh, and S. K. Chang, "A multiscale model for bioimpedance dispersion of liver tissue," IEEE Transactions on Biomedical Engineering, vol. 59, no. 6, pp. 1593-1597, 2012.

[88] M. A. Callejón, P. del Campo, J. Reina-Tosina, and L. M. Roa, "A parametric computational analysis into galvanic coupling intrabody communication," IEEE Journal of Biomedical and Health Informatics, vol. 22, no. 4, pp. 1087-1096, 2018.

[89] B. Taji, S. Shirmohammadi, V. Groza, and I. Batkin, "Impact of skin-electrode interface on electrocardiogram measurements using conductive textile electrodes," IEEE Transactions on Instrumentation and Measurement, vol. 63, no. 6, pp. 1412-1422, 2014.

[90] M. H. Jones and J. Scott, "Scaling of electrode-electrolyte interface model parameters in phosphate buffered saline," IEEE Transactions on Biomedical Circuits and Systems, vol. 9, no. 3, pp. 441-448, 2015.

[91] H. H. Woltjer, B. J. M. van der Meer, H. J. Bogaard, and P. M. J. M. de Vries, "Comparison between spot and band electrodes and between two equations for calculations of stroke volume by means of impedance cardiography," Medical \& Biological Engineering \& Computing, vol. 33, no. 3, pp. 330-334, 1995.

[92] B. Eilebrecht, J. Willkomm, A. Pohl, T. Wartzek, and S. Leonhardt, "Impedance measurement system for determination of capacitive electrode coupling," IEEE Transactions on Biomedical Circuits and Systems, vol. 7, no. 5, pp. 682-689, 2013.

[93] M. Guermandi, R. Cardu, E. F. Scarselli, and R. Guerrieri, "Active electrode IC for EEG and electrical impedance tomography with continuous monitoring of contact impedance," IEEE Transactions on Biomedical Circuits and Systems, vol. 9, no. 1, pp. 21-33, 2015.

[94] B. W. Lee, C. Lee, J. Kim, and M. Lee, "Comparison of conductive fabric electrode with electromyography to evaluate knee joint movement," IEEE Sensors Journal, vol. 12, no. 2, pp. 410-411, 2012.

[95] J. Ferreira, I. Pau, K. Lindecrantz, and F. Seoane, "A handheld and textile-enabled bioimpedance system for ubiquitous body composition analysis. an initial functional validation," IEEE Journal of Biomedical and Health Informatics, vol. 21, no. 5, pp. 1224-1232, 2017.

[96] Y. Yu, K. A. Al Mamun, A. S. Shanta, S. K. Islam, and N. McFarlane, "Vertically aligned carbon nanofibers as a cell impedance sensor," IEEE Transactions on Nanotechnology, vol. 15, no. 6, pp. 856-861, 2016.

[97] G. Qiao, W. Wang, W. Duan, F. Zheng, A. J. Sinclair, and C. R. Chatwin, "Bioimpedance analysis for the characterization of breast cancer cells in suspension," IEEE Transactions on Biomedical Engineering, vol. 59, no. 8, pp. 2321-2329, 2012.
[98] H. Kwon, H. Wi, B. Karki et al., "Bioimpedance spectroscopy tensor probe for anisotropic measurements," IEEE Electronics Letters, vol. 48, no. 20, pp. 1253-1255, 2012.

[99] H. Kwon, M. Guasch, J. A. Nagy, S. B. Rutkove, and B. Sanchez, "New electrical impedance methods for the in situ measurement of the complex permittivity of anisotropic skeletal muscle using multipolar needles," Scientific Reports, vol. 9, no. 1, 2019.

[100] B. Karki, H. Wi, A. McEwan et al., "Evaluation of a multielectrode bioimpedance spectroscopy tensor probe to detect the anisotropic conductivity spectra of biological tissues," Measurement Science and Technology, vol. 25, no. 7, 2014.

[101] D. Teichmann, L. Rohe, A. Niesche, M. Mueller, K. Radermacher, and S. Leonhardt, "Estimation of penetrated bone layers during craniotomy via bioimpedance measurement," IEEE Transactions on Biomedical Engineering, vol. 64, no. 4, pp. 765-774, 2017.

[102] A. S. Tucker, R. M. Fox, and R. J. Sadleir, "Biocompatible, high precision, wideband, improved howland current source with lead-lag compensation," IEEE Transactions on Biomedical Circuits and Systems, vol. 7, no. 1, pp. 63-70, 2013.

[103] A. S. Anusha, S. P. Preejith, J. Joseph, and M. Sivaprakasam, "Design and implementation of a hand-to-hand multifrequency bioimpedance measurement scheme for Total Body Water estimation," in Proceedings of the 2017 IEEE International Instrumentation and Measurement Technology Conference, I2MTC 2017, May 2017.

[104] H. Ko, T. Lee, J. Kim, J. Park, and J. P. Kim, "Ultralowpower bioimpedance IC with intermediate frequency shifting chopper," IEEE Transactions on Circuits and Systems II: Express Briefs, vol. 63, no. 3, pp. 259-263, 2016.

[105] M. Etemadi, P. Chung, J. A. Heller, J. A. Liu, L. Rand, and S. Roy, "Towards birthalert-a clinical device intended for early preterm birth detection," IEEE Transactions on Biomedical Engineering, vol. 60, no. 12, pp. 3484-3493, 2013.

[106] J. E. Sanders, M. A. Moehring, T. M. Rothlisberger et al., "A bioimpedance analysis platform for amputee residual limb assessment," IEEE Transactions on Biomedical Engineering, vol. 63, no. 8, pp. 1760-1770, 2016.

[107] L. Yan, J. Pettine, S. Mitra et al., "A 13 murmA analog signal processing IC for accurate recognition of multiple intra-cardiac signals," IEEE Transactions on Biomedical Circuits and Systems, vol. 7, no. 6, pp. 785-795, 2013.

[108] R. Harder, A. Diedrich, J. S. Whitfield, M. S. Buchowski, J. B. Pietsch, and F. J. Baudenbacher, "Smart multi-frequency bioelectrical impedance spectrometer for BIA and BIVA applications," IEEE Transactions on Biomedical Circuits and Systems, vol. 10, no. 4, pp. 912-919, 2016.

[109] L. Constantinou, I. F. Triantis, R. Bayford, and A. Demosthenous, "High-power CMOS current driver with accurate transconductance for electrical impedance tomography," IEEE Transactions on Biomedical Circuits and Systems, vol. 8, no. 4, pp. 575-583, 2014.

[110] P. J. Langlois, N. Neshatvar, and A. Demosthenous, "A sinusoidal current driver with an extended frequency range and multifrequency operation for bioimpedance applications," IEEE Transactions on Biomedical Circuits and Systems, vol. 9, no. 3, pp. 401-411, 2015.

[111] L. Constantinou, R. Bayford, and A. Demosthenous, "A wideband low-distortion CMOS current driver for tissue impedance analysis," IEEE Transactions on Circuits and Systems II: Express Briefs, vol. 62, no. 2, pp. 154-158, 2015. 
[112] V. Chitturi and N. Farrukh, "An alternate voltage-controlled current source for electrical impedance tomography applications," Lecture Notes in Networks and Systems, vol. 43, pp. 93$102,2019$.

[113] Ø. G. Martinsen, B. Nordbotten, S. Grimnes, H. Fossan, and J. Eilevstjønn, "Bioimpedance-based respiration monitoring with a defibrillator," IEEE Transactions on Biomedical Engineering, vol. 61, no. 6, pp. 1858-1862, 2014.

[114] A. Yúfera and A. Rueda, "Design of a CMOS closed-loop system with applications to bio-impedance measurements," Microelectronics Journal, vol. 41, no. 4, pp. 231-239, 2010.

[115] P. Kassanos, I. F. Triantis, and A. Demosthenous, "A CMOS magnitude/phase measurement chip for impedance spectroscopy," IEEE Sensors Journal, vol. 13, no. 6, pp. 2229-2236, 2013.

[116] S.-J. Kweon, J.-H. Park, S. Shin, S.-S. Yoo, and H.-J. Yoo, "A reconfigurable time-to-digital converter based on time stretcher and chain-delay-line for electrical bioimpedance spectroscopy," in Proceedings of the 60th IEEE International Midwest Symposium on Circuits and Systems, MWSCAS 2017, vol. 2017, pp. 10371040, August 2017.

[117] B. Sanchez, G. Vandersteen, R. Bragos, and J. Schoukens, “Basics of broadband impedance spectroscopy measurements using periodic excitations," Measurement Science and Technology, vol. 23, no. 10, 2012.

[118] M. Min, T. Parve, A. Ronk, P. Annus, and T. Paavle, "Synchronous sampling and demodulation in an instrument for multifrequency bioimpedance measurement," IEEE Transactions on Instrumentation and Measurement, vol. 56, no. 4, pp. 1365-1372, 2007.

[119] S. K. Oruganti, S. H. Heo, H. Ma, and F. Bien, "Wireless energy transfer: Touch/Proximity/Hover sensing for large contoured displays and industrial applications," IEEE Sensors Journal, vol. 15, no. 4, pp. 2062-2068, 2015.

[120] F. R. Parente, S. Di Giovanni, G. Ferri, V. Stornelli, G. Pennazza, and M. Santonico, "An analog bootstrapped biosignal read-out circuit with common-mode impedance two-electrode compensation," IEEE Sensors Journal, vol. 18, no. 7, pp. 2861-2869, 2018.

[121] N. Li, H. Xu, W. Wang, Z. Zhou, G. Qiao, and D. D.-U. Li, "A high-speed bioelectrical impedance spectroscopy system based on the digital auto-balancing bridge method," Measurement Science and Technology, vol. 24, no. 6, 2013.

[122] J. He, M. Wang, X. Li, G. Li, and L. Lin, "Pulse wave detection method based on the bio-impedance of the wrist," Review of Scientific Instruments, vol. 87, no. 5, 2016.

[123] Y. Liu, X. Qiao, G. Li, and L. Lin, "An improved device for bioimpedance deviation measurements based on 4-electrode half bridge," Review of Scientific Instruments, vol. 87, no. 10, 2016.

[124] J. C. Bæg, H. Wi, T. I. Oh, A. L. McEwan, and E. J. Woo, "An amplitude-to-time conversion technique suitable for multichannel data acquisition and bioimpedance imaging," IEEE Transactions on Biomedical Circuits and Systems, vol. 7, no. 3, pp. 349-354, 2013.

[125] B. Sanchez, G. Vandersteen, R. Bragos, and J. Schoukens, "Optimal multisine excitation design for broadband electrical impedance spectroscopy," Measurement Science and Technology, vol. 22, no. 11, 2011.

[126] M. Min and T. Paavle, "Broadband discrete-level excitations for improved extraction of information in bioimpedance measurements," Physiological Measurement, vol. 35, no. 6, pp. 997-1010, 2014.
[127] T. Paavle, M. Min, and T. Parve, "Using of chirp excitation for bioimpedance estimation: Theoretical aspects and modeling," in Proceedings of the 11th Biennial Baltic Electronics Conference, BEC 2008, pp. 325-328, October 2008.

[128] B. Sanchez, J. Schoukens, R. Bragos, and G. Vandersteen, "Novel estimation of the electrical bioimpedance using the local polynomial method. application to in vivo real-time myocardium tissue impedance characterization during the cardiac cycle," IEEE Transactions on Biomedical Engineering, vol. 58, no. 12, part 1, pp. 3376-3385, 2011.

[129] X. Shi, F. You, Z. Ji, F. Fu, R. Liu, and X. Dong, "Digital Demodulation in data acquisition system for multi-frequency electrical impedance tomography," in Proceedings of the 4th International Conference on Bioinformatics and Biomedical Engineering, iCBBE 2010, June 2010.

[130] X. Shi, F. You, C. Xu et al., "Design and implementation of a high-precision electrical impedance tomography data acquisition system for brain imaging," in Proceedings of the 12th IEEE Biomedical Circuits and Systems Conference, BioCAS 2016, pp. 9-13, China, October 2016.

[131] J. Gracia, V. P. Seppa, J. Viik, and J. Hyttinen, "Multilead measurement system for the time-domain analysis of bioimpedance magnitude," IEEE Transactions on Biomedical Engineering, vol. 59, no. 8, pp. 2273-2280, 2012.

[132] F. Seoane, J. Ferreira, J. J. Sanchéz, and R. Bragós, "An analog front-end enables electrical impedance spectroscopy system on-chip for biomedical applications," Physiological Measurement, vol. 29, no. 6, pp. S267-S278, 2008.

[133] B. Sanchez, A. L. Aroul, E. Bartolome, K. Soundarapandian, and R. Bragos, "Propagation of measurement errors through body composition equations for body impedance analysis," IEEE Transactions on Instrumentation and Measurement, vol. 63, no. 6, pp. 1535-1544, 2014.

[134] M. Konijnenburg, S. Stanzione, L. Yan et al., "A Multi(bio)sensor Acquisition System with Integrated Processor, Power Management, $8 \times 8$ LED Drivers, and Simultaneously Synchronized ECG, BIO-Z, GSR, and Two PPG Readouts," IEEE Journal of Solid-State Circuits, vol. 51, no. 11, pp. 2584-2595, 2016.

[135] E. M. Bartels, E. R. Sørensen, and A. P. Harrison, "Multifrequency bioimpedance in human muscle assessment," Physiological Reports, vol. 3, no. 4, 2015.

[136] R. Kubendran, S. Lee, S. Mitra, and R. F. Yazicioglu, "Error correction algorithm for high accuracy bio-impedance measurement in wearable healthcare applications," IEEE Transactions on Biomedical Circuits and Systems, vol. 8, no. 2, pp. 196-205, 2014.

[137] A. A. Silverio, W.-Y. Chung, and V. F. S. Tsai, "A low power high CMRR CMOS instrumentation amplifier for Bio-impedance Spectroscopy," in Proceedings of the 2014 IEEE International Symposium on Bioelectronics and Bioinformatics, IEEE ISBB 2014, April 2014.

[138] E. H. Sanabria, J. A. Palacio, H. H. Herrera, and W. Van Noije, "A design methodology for an integrated CMOS instrumentation amplifier for bioespectroscopy applications," in Proceedings of the 2017 CHILEAN Conference on Electrical, Electronics Engineering, Information and Communication Technologies, CHILECON 2017, pp. 1-7, October 2017.

[139] K. Kim, K. Song, K. Bong et al., "A $24 \mu \mathrm{W} 38.51 \mathrm{~m} \Omega \mathrm{rms}$ resolution bio-impedance sensor with dual path instrumentation amplifier," in Proceedings of the 43rd IEEE European Solid State Circuits Conference, ESSCIRC 2017, pp. 223-226, September 2017. 
[140] M. Zamani, Y. Rezaeiyan, O. Shoaei, and W. A. Serdijn, "A $1.55 \mu \mathrm{w}$ bio-impedance measurement system for implantable cardiac pacemakers in $0.18 \mu \mathrm{m}$ CMOS," IEEE Transactions on Biomedical Circuits and Systems, vol. 12, no. 1, pp. 211-221, 2018.

[141] M. Ulbrich, J. Muhlsteff, D. Teichmann, S. Leonhardt, and M. Walter, "A thorax simulator for complex dynamic bioimpedance measurements with textile electrodes," IEEE Transactions on Biomedical Circuits and Systems, vol. 9, no. 3, pp. 412-420, 2015.

[142] C. Aliau-Bonet and R. Pallas-Areny, "A novel method to estimate body capacitance to ground at mid frequencies," IEEE Transactions on Instrumentation and Measurement, vol. 62, no. 9, pp. 2519-2525, 2013.

[143] C. Aliau-Bonet and R. Pallas-Areny, "On the effect of body capacitance to ground in tetrapolar bioimpedance measurements," IEEE Transactions on Biomedical Engineering, vol. 59, no. 12, pp. 3405-3411, 2012.

[144] Y. Yang, W. Ni, Q. Sun, H. Wen, and Z. Teng, "Improved Cole parameter extraction based on the least absolute deviation method," Physiological Measurement, vol. 34, no. 10, pp. 12391252, 2013.

[145] R. Buendia, R. Gil-Pita, and F. Seoane, "Cole parameter estimation from total right side electrical bioimpedance spectroscopy measurements-influence of the number of frequencies and the upper limit," in Proceedings of the 33rd Annual International Conference of the IEEE Engineering in Medicine and Biology Society (EMBS '11), pp. 1843-1846, IEEE, Boston, Mass, USA, September 2011.

[146] T. J. Freeborn, B. Maundy, and A. Elwakil, "Improved ColeCole parameter extraction from frequency response using least squares fitting," in Proceedings of the 2012 IEEE International Symposium on Circuits and Systems, ISCAS 2012, pp. 337-340, Republic of Korea, May 2012.

[147] L. C. Ward, T. Essex, and B. H. Cornish, "Determination of Cole parameters in multiple frequency bioelectrical impedance analysis using only the measurement of impedances," Physiological Measurement, vol. 27, no. 9, pp. 839-850, 2006.

[148] B. J. Nordbotten, C. Tronstad, Ø. G. Martinsen, and S. Grimnes, "Evaluation of algorithms for calculating bioimpedance phase angle values from measured whole-body impedance modulus," Physiological Measurement, vol. 32, no. 7, pp. 755-765, 2011.

[149] A. Keshtkar, Z. Salehnia, A. Keshtkar, and B. Shokouhi, "Bladder cancer detection using electrical impedance technique (Tabriz Mark 1)," Pathology Research International, vol. 2012, Article ID 470101, 5 pages, 2012.

[150] F. Villa, A. Magnani, G. Merati, and P. Castiglioni, "Feasibility of long-term monitoring of multifrequency and multisegment body impedance by portable devices," IEEE Transactions on Biomedical Engineering, vol. 61, no. 6, pp. 1877-1886, 2014.

[151] S. L. Yoon, O. Grundmann, J. J. Williams, L. Gordan, and T. J. George, "Body composition changes differ by gender in stomach, colorectal, and biliary cancer patients with cachexia: Results from a pilot study, Cancer Medicine, vol. 7, no. 8, pp. 3695-3703, 2018.

[152] C. Earthman, D. Traughber, J. Dobratz, and W. Howell, "Bioimpedance spectroscopy for clinical assessment of fluid distribution and body cell mass," Nutrition in Clinical Practice, vol. 22, no. 4, pp. 389-405, 2007.

[153] S. Sipahi, E. Hur, S. Demirtas et al., "Body composition monitor measurement technique for the detection of volume status in peritoneal dialysis patients: the effect of abdominal fullness," International Urology and Nephrology, vol. 43, no. 4, pp. 11951199, 2011.
[154] U. M. Moissl, P. Wabel, P. W. Chamney et al., "Body fluid volume determination via body composition spectroscopy in health and disease," Physiological Measurement, vol. 27, no. 9, pp. 921-933, 2006.

[155] S. Khalil, M. Mohktar, and F. Ibrahim, "The theory and fundamentals of bioimpedance analysis in clinical status monitoring and diagnosis of diseases," Sensors, vol. 14, no. 6, pp. 1089510928, 2014.

[156] R. Thibault and C. Pichard, "The evaluation of body composition: a useful tool for clinical practice," Annals of Nutrition and Metabolism, vol. 60, no. 1, pp. 6-16, 2012.

[157] P. P. Gupta, G. C. Fonarow, and T. B. Horwich, "Obesity and the obesity paradox in heart failure," Canadian Journal of Cardiology, vol. 31, no. 2, pp. 195-202, 2015.

[158] S. Hocking, D. Samocha-Bonet, K.-L. Milner, J. R. Greenfield, and D. J. Chisholm, "Adiposity and insulin resistance in humans: the role of the different tissue and cellular lipid depots," Endocrine Reviews, vol. 34, no. 4, pp. 463-500, 2013.

[159] M. Kovarik, M. Hronek, and Z. Zadak, "Clinically relevant determinants of body composition, function and nutritional status as mortality predictors in lung cancer patients," Lung Cancer, vol. 84, no. 1, pp. 1-6, 2014.

[160] E. De Rosa, L. Santarpia, M. Marra et al., "Preliminary evaluation of the prevalence of sarcopenia in obese patients from Southern Italy," Nutrition Journal, vol. 31, no. 1, pp. 79-83, 2015.

[161] E. M. Widen and D. Gallagher, "Body composition changes in pregnancy: measurement, predictors and outcomes," European Journal of Clinical Nutrition, vol. 68, no. 6, pp. 643-652, 2014.

[162] M. L. Giannì, P. Roggero, A. Orsi et al., "Body composition changes in the first 6 months of life according to method of feeding," Journal of Human Lactation, vol. 30, no. 2, pp. 148-155, 2014.

[163] A. Shuster, M. Patlas, J. H. Pinthus, and M. Mourtzakis, “The clinical importance of visceral adiposity: a critical review of methods for visceral adipose tissue analysis," British Journal of Radiology, vol. 85, no. 1009, pp. 1-10, 2012.

[164] A. A. Jackson, M. Johnson, K. Durkin, and S. Wootton, "Body composition assessment in nutrition research: value of BIA technology," European Journal of Clinical Nutrition, vol. 67, no. S1, pp. S71-S78, 2013.

[165] C. M. Toomey, A. Cremona, K. Hughes, C. Norton, and P. Jakeman, "A review of body composition measurement in the assessment of health," Topics in Clinical Nutrition, vol. 30, no. 1, pp. 16-32, 2015.

[166] K. Cumming, G. E. Hoyle, J. D. Hutchison, and R. L. Soiza, "Bioelectrical impedance analysis is more accurate than clinical examination in determining the volaemic status of elderly patients with fragility fracture and hyponatraemia," The Journal of Nutrition, Health \& Aging, vol. 18, no. 8, pp. 744-750, 2014.

[167] C. Tsigos, C. Stefanaki, G. I. Lambrou, D. Boschiero, and G. P. Chrousos, "Stress and inflammatory biomarkers and symptoms are associated with bioimpedance measures," European Journal of Clinical Investigation, vol. 45, no. 2, pp. 126-134, 2015.

[168] L. Mattar, N. Godart, J. C. Melchior, and C. Pichard, "Anorexia nervosa and nutritional assessment: contribution of body composition measurements," Nutrition Research Reviews, vol. 24, no. 1, pp. 39-45, 2011.

[169] A. F. Casey, "Measuring body composition in individuals with intellectual disability: A scoping review," Journal of Obesity, vol. 2013, Article ID 628428, 6 pages, 2013. 
[170] D. Thangarajah, M. J. Hyde, V. K. S. Konteti, S. Santhakumaran, G. Frost, and J. M. E. Fell, "Systematic review: Body composition in children with inflammatory bowel disease," Alimentary Pharmacology \& Therapeutics, vol. 42, no. 2, pp. 142-157, 2015.

[171] E. Haverkort, P. Reijven, J. Binnekade et al., "Bioelectrical impedance analysis to estimate body composition in surgical and oncological patients: a systematic review," European Journal of Clinical Nutrition, vol. 69, pp. 3-13, 2015.

[172] Z. Erdogan Iyigun, D. Selamoglu, G. Alco et al., "Bioelectrical impedance for detecting and monitoring lymphedema in patients with breast cancer. Preliminary results of the florence nightingale breast study group," Lymphatic Research and Biology, vol. 13, no. 1, pp. 40-45, 2015.

[173] B. E. Lingwood, "Bioelectrical impedance analysis for assessment of fluid status and body composition in neonates-the good, the bad and the unknown," European Journal of Clinical Nutrition, vol. 67, no. S1, pp. S28-S33, 2013.

[174] E. Rivers, B. Nguyen, S. Havstad et al., "Early goal-directed therapy in the treatment of severe sepsis and septic shock," The New England Journal of Medicine, vol. 345, no. 19, pp. 1368-1377, 2001.

[175] J. H. Boyd, J. Forbes, T. Nakada, K. R. Walley, and J. A. Russell, "Fluid resuscitation in septic shock: a positive fluid balance and elevated central venous pressure are associated with increased mortality," Critical Care Medicine, vol. 39, no. 2, pp. 259-265, 2011.

[176] H. P. Wiedemann, A. P. Wheeler, G. R. Bernard et al., "Comparison of two fluid-management strategies in acute lung injury," The New England Journal of Medicine, vol. 354, no. 24, pp. 2564$2575,2006$.

[177] U. Mulasi, A. J. Kuchnia, A. J. Cole, and C. P. Earthman, "Bioimpedance at the bedside: current applications, limitations, and opportunities," Nutrition in Clinical Practice, vol. 30, no. 2, pp. 180-193, 2015.

[178] C. Moctezuma-Velázquez, I. García-Juárez, R. Soto-Solís, J. Hernández-Cortés, and A. Torre, "Nutritional assessment and treatment of patients with liver cirrhosis," Nutrition Journal, vol. 29, no. 11-12, pp. 1279-1285, 2013.

[179] M. Siervo, C. Prado, L. Hooper et al., "Serum osmolarity and haematocrit do not modify the association between the impedance index $(\mathrm{Ht} 2 / \mathrm{Z})$ and total body water in the very old: The Newcastle 85+ Study," Archives of Gerontology and Geriatrics, vol. 60, no. 1, pp. 227-232, 2015.

[180] S. M. Ribeiro and J. J. Kehayias, "Sarcopenia and the analysis of body composition," Advances in Nutrition, vol. 5, no. 3, pp. 260-267, 2014.

[181] G. Mascherini, C. Petri, and G. Galanti, "Integrated total body composition and localized fat-free mass assessment," Sport Sciences for Health, vol. 11, no. 2, pp. 217-225, 2015.

[182] A. Walter-Kroker, A. Kroker, M. Mattiucci-Guehlke, and T. Glaab, "A practical guide to bioelectrical impedance analysis using the example of chronic obstructive pulmonary disease," Nutrition Journal, vol. 10, no. 1, 2011.

[183] A. M. Abbatecola, A. Fumagalli, L. Spazzafumo et al., "Body composition markers in older persons with COPD," Age and Ageing, vol. 43, no. 4, pp. 548-553, 2014.

[184] J. Bauer, E. Egan, and A. Clavarino, "The scored patientgenerated subjective global assessment is an effective nutrition assessment tool in subjects with chronic obstructive pulmonary disease," e-SPEN Journal, vol. 6, no. 1, pp. e27-e30, 2011.
[185] S. Mitra, "Extracellular hydration, cardiovascular risk, and the interstitium: a three-dimensional view," Kidney International, vol. 85, no. 3, pp. 510-512, 2014.

[186] R. Hussain, F. Kappel, F. Zhu, N. W. Levin, and P. Kotanko, "Body composition and solute kinetics in hemodialysis patients: a mathematical model," IET Communications, vol. 6, no. 18, pp. 3301-3308, 2012.

[187] R. Patterson, "Body fluid determinations using multiple impedance measurements," IEEE Engineering in Medicine and Biology Magazine, vol. 8, no. 1, pp. 16-18, 1989.

[188] S. S. Sun, W. C. Chumlea, S. B. Heymsfield et al., "Development of bioelectrical impedance analysis prediction equations for body composition with the use of a multicomponent model for use in epidemiologic surveys," American Journal of Clinical Nutrition, vol. 77, no. 2, pp. 331-340, 2003.

[189] B. L. Heitmann, "Prediction of body water and fat in adult Danes from measurement of electrical impedance. A validation study," International Journal of Obesity, vol. 14, no. 9, pp. 789-802, 1990.

[190] G. Sergi, M. Bussolotto, P. Perini et al., "Accuracy of bioelectrical impedance analysis in estimation of extracellular space in healthy subjects and in fluid retention states," Annals of Nutrition and Metabolism, vol. 38, no. 3, pp. 158-165, 1994.

[191] P. Deurenberg, K. Van Der Kooy, R. Leenen, J. A. Weststrate, and J. C. Seidell, "Sex and age specific prediction formulas for estimating body composition from bioelectrical impedance: A cross-validation study," International Journal of Obesity, vol. 15, no. 1, pp. 17-25, 1991.

[192] M. Ring, C. Lohmueller, M. Rauh, J. Mester, and B. M. Eskofier, "A temperature-based bioimpedance correction for water loss estimation during sports," IEEE Journal of Biomedical and Health Informatics, vol. 20, no. 6, pp. 1477-1484, 2016.

[193] F. Seoane, S. Abtahi, F. Abtahi et al., "Mean expected error in prediction of total body water: A true accuracy comparison between bioimpedance spectroscopy and single frequency regression equations," BioMed Research International, vol. 2015, Article ID 656323, 11 pages, 2015.

[194] U. G. Kyle, I. Bosaeus, A. D. De Lorenzo et al., "Bioelectrical impedance analysis-part II: utilization in clinical practice," Clinical Nutrition, vol. 23, no. 6, pp. 1430-1453, 2004.

[195] L. Nescolarde, T. Doñate, A. Piccoli, and J. Rosell, "Comparison of segmental with whole-body impedance measurements in peritoneal dialysis patients," Medical Engineering \& Physics, vol. 30, no. 7, pp. 817-824, 2008.

[196] M. D. Van Loan, P. Withers, J. Matthie, and P. L. Mayclin, "Use of bioimpedance spectroscopy to determine extracellular fluid, intracellular fluid, total body water, and fat-free mass," Basic Life Sciences, vol. 60, pp. 67-70, 1993.

[197] M. Y. Jaffrin and M. V. Moreno, "Measurements of total body water with a foot-to-foot impedancemeter," Medical Engineering \& Physics, vol. 30, no. 4, pp. 483-489, 2008.

[198] M. Y. Jaffrin and H. Morel, "Body fluid volumes measurements by impedance: a review of bioimpedance spectroscopy (BIS) and bioimpedance analysis (BIA) methods," Medical Engineering \& Physics, vol. 30, no. 10, pp. 1257-1269, 2008.

[199] J. Eisenkölbl, M. Kartasurya, and K. Widhalm, "Underestimation of percentage fat mass measured by bioelectrical impedance analysis compared to dual energy X-ray absorptiometry method in obese children," European Journal of Clinical Nutrition, vol. 55, no. 6, pp. 423-429, 2001.

[200] A. Piccoli, B. Rossi, L. Pillon, and G. Bucciante, "A new method for monitoring body fluid variation by bioimpedance analysis: 
the RXc graph," Kidney International, vol. 46, no. 2, pp. 534-539, 1994.

[201] L. Nescolarde, A. Piccoli, A. Román et al., "Bioelectrical impedance vector analysis in haemodialysis patients: relation between oedema and mortality," Physiological Measurement, vol. 25, no. 5, pp. 1271-1280, 2004.

[202] M. D. L. Á. Espinosa-Cuevas, L. Rivas-Rodríguez, E. C. González-Medina, X. Atilano-Carsi, P. Miranda-Alatriste, and R. Correa-Rotter, "Bioimpedance vector analysis for body composition in Mexican population," Revista de Investigación Clínica, vol. 59, no. 1, pp. 15-24, 2007.

[203] A. Piccoli, L. Pillon, and F. Dumler, "Impedance vector distribution by sex, race, body mass index, and age in the United States: standard reference intervals as bivariate Z scores," Nutrition Journal, vol. 18, no. 2, pp. 153-167, 2002.

[204] M. Peppa, C. Stefanaki, A. Papaefstathiou, D. Boschiero, G. Dimitriadis, and G. P. Chrousos, "Bioimpedance analysis vs. DEXA as a screening tool for osteosarcopenia in lean, overweight and obese caucasian postmenopausal females," Hormones, vol. 16, no. 2, pp. 181-193, 2017.

[205] A. Molfino, B. R. Don, and G. A. Kaysen, "Comparison of bioimpedance and dual-energy X-ray absorptiometry for measurement of fat mass in hemodialysis patients," Nephron Clinical Practice, vol. 122, no. 3-4, pp. 127-133, 2012.

[206] M. T. Najafi, O. Nasiri, A. Alamdari et al., "Comparison of body composition assessed by multi-frequency segmental bioelectrical impedance analysis and dual energy X-ray absorptiometry in hemodialysis patients," Nephro-Urology Monthly, vol. 10, no. 6, 2018.

[207] P. S. Lim, C. H. Chen, F. Zhu et al., "Validating body fat assessment by bioelectric impedance spectroscopy in taiwanese hemodialysis patients," Journal of Renal Nutrition, vol. 27, no. 1, pp. 37-44, 2017.

[208] P. Deurenberg, A. Tagliabue, and F. J. M. Schouten, "Multifrequency impedance for the prediction of extracellular water and total body water," British Journal of Nutrition, vol. 73, no. 3, pp. 349-358, 1995.

[209] R. F. Kushner and D. A. Schoeller, "Estimation of total body water by bioelectrical impedance analysis," American Journal of Clinical Nutrition, vol. 44, no. 3, pp. 417-424, 1986.

[210] B. C. Wingo, V. G. Barry, A. C. Ellis, and B. A. Gower, "Comparison of segmental body composition estimated by bioelectrical impedance analysis and dual-energy X-ray absorptiometry," Clinical Nutrition ESPEN, vol. 28, pp. 141-147, 2018.

[211] J. G. Raimann, F. Zhu, J. Wang et al., "Comparison of fluid volume estimates in chronic hemodialysis patients by bioimpedance, direct isotopic, and dilution methods," Kidney International, vol. 85, no. 4, pp. 898-908, 2014.

[212] R. Větrovská, Z. Vilikus, J. Klaschka et al., "Does impedance measure a functional state of the body fat?" Physiological Research, vol. 63, no. 2, pp. S309-S320, 2014.

[213] Y. Zhou, P. Höglund, and N. Clyne, "Comparison of DEXA and bioimpedance for body composition measurements in nondialysis patients with ckd," Journal of Renal Nutrition, vol. 29, no. 1, pp. 33-38, 2019.

[214] M. Krzykała, J. Konarski, R. Malina, K. Rachwalski, P. Leszczyński, and E. Ziółkowska-Łajp, "Fatness of female field hockey players: Comparison of estimates with different methods," Homo: Journal of Comparative Human Biology, vol. 67, no. 3, pp. 245-257, 2016.
[215] R. Patterson, "Fundamentals of impedance cardiography," IEEE Engineering in Medicine and Biology Magazine, vol. 8, no. 1, pp. 35-38, 1989.

[216] A. Sherwood(Chair), M. T. Allen, J. Fahrenberg, R. M. Kelsey, W. R. Lovallo, and L. J. Doornen, "Methodological guidelines for impedance cardiography," Psychophysiology, vol. 27, no. 1, pp. $1-23,1990$.

[217] S. Benouar, A. Hafid, M. Attari, M. Kedir-Talha, and F. Seoane, "Systematic variability in ICG recordings results in ICG complex subtypes - steps towards the enhancement of ICG characterization," Journal of Electrical Bioimpedance (JEB), vol. 9, no. 1, pp. 72-82, 2018.

[218] G. Singh, S. Anand, B. Lall, A. Srivastava, and V. Singh, "A technical review of various bioelectric impedance methods for health monitoring," in Proceedings of the 2018 IEEE Long Island Systems, Applications and Technology Conference, LISAT 2018, pp. 1-6, 2018.

[219] T. K. Bera, "Bioelectrical impedance methods for noninvasive health monitoring: A review," Journal of Medical Engineering, vol. 2014, Article ID 381251, 28 pages, 2014.

[220] J. Malmivuo and R. Plonsey, Bioelectromagnetism: Principles and Applications of Bioelectric and Biomagnetic Fields, Oxford University Press, New York, NY, USA, 1995.

[221] X. Chen, X. Hu, R. Ren et al., "Noninvasive ambulatory monitoring of the electric and mechanical function of heart with a multifunction wearable sensor," in Proceedings of the 2014 IEEE 38th International Computer Software and Applications Conference Workshops, pp. 662-667, Vasteras, Sweden, July 2014.

[222] J. Xu, P. Harpe, and C. V. Hoof, "An energy-efficient and reconfigurable sensor IC for bio-impedance spectroscopy and ECG recording," IEEE Journal on Emerging and Selected Topics in Circuits and Systems, vol. 8, no. 3, pp. 616-626, 2018.

[223] X. Chen, J. Xie, Z. Fang, and S. Xia, "Low power electrocardiography and impedance cardiography detection system based on LabVIEW and Bluetooth Low Energy," in Proceedings of the 2015 IET International Conference on Biomedical Image and Signal Processing, ICBISP 2015, 2015.

[224] M. Snajdarova, S. Borik, and I. Cap, "Features extraction from impedance cardiography signal," in Proceedings of the 11th International Conference on Measurement, pp. 225-228, May 2017.

[225] X. Hu, X. Chen, and R. Ren, "Noninvasive ambulatory hemodynamic monitoring based on electrocardiogram and impedance cardiography," Journal of Fiber Bioengineering and Informatics, vol. 8, no. 4, pp. 741-749, 2015.

[226] H. Yazdanian, A. Mahnam, M. Edrisi, and M. Esfahani, "Design and implementation of a portable impedance cardiography system for noninvasive stroke volume monitoring," Journal of Medical Signals and Sensors, vol. 6, no. 1, pp. 47-56, 2016.

[227] M. Ulbrich, J. Mühlsteff, A. Sipilä et al., "The IMPACT shirt: textile integrated and portable impedance cardiography," Physiological Measurement, vol. 35, no. 6, pp. 1181-1196, 2014.

[228] A. Szczesna, A. Nowak, P. Grabiec, P. Rozentryt, and M. Wojciechowska, "Innovations in biomedical engineering. advances in intelligent systems and computing," in Wearable sensor vest design study for vital parameters measurement system, Advances in Intelligent Systems and Computing, vol. 526, pp. 330-337, Springer, Cham, Switzerland, 2017.

[229] M. Banet, S. Pede, M. Dhillon, and R. Hunt, Body Worn Sensor for Characterizing Patients with Heart Failure, Perminova Inc., 2014, Patent US 2014/0187990 A1. 
[230] E. Pinheiro, O. Postolache, and P. Girão, "Contactless impedance cardiography using embedded sensors," Measurement Science Review, vol. 13, no. 3, pp. 157-164, 2013.

[231] D. B. Jain, J. Weeks, D. Nadezhdin, and J.-F. Asselin, "Contactless electric cardiogram system," Patent WO 2016/044933 Al, Article ID 044933, 2016.

[232] S.-H. Liu, J.-J. Wang, S. Chun-Hung, and D.-C. Cheng, "Improvement of left ventricular ejection time measurement in the impedance cardiography combined with the reflection photoplethysmography," Sensors, vol. 18, no. 9, 2018.

[233] M. Cieslak, W. S. Ryan, A. Macy et al., "Simultaneous acquisition of functional magnetic resonance images and impedance cardiography," Psychophysiology, vol. 52, no. 4, pp. 481-488, 2015.

[234] M. Forouzanfar, F. C. Baker, M. de Zambotti, C. McCall, L. Giovangrandi, and G. T. A. Kovacs, "Toward a better noninvasive assessment of preejection period: A novel automatic algorithm for B-point detection and correction on thoracic impedance cardiogram," Psychophysiology, vol. 55, no. 8, Article ID e13072, 2018.

[235] J. R. Árbol, P. Perakakis, A. Garrido, J. L. Mata, M. C. FernándezSantaella, and J. Vila, "Mathematical detection of aortic valve opening (B point) in impedance cardiography: A comparison of three popular algorithms," Psychophysiology, vol. 54, no. 3, pp. 350-357, 2017.

[236] S. S. Mirza and M. Z. U. Rahman, "Efficient adaptive filtering techniques for thoracic electrical bio-impedance analysis in health care systems," Journal of Medical Imaging and Health Informatics, vol. 7, no. 6, pp. 1126-1138, 2017.

[237] S. Liu, K. Yue, H. Yang, L. Liu, X. Duan, and T. Guo, "Study on cardiac impedance signal feature point extraction," in Proceedings of the 3rd IEEE Information Technology and Mechatronics Engineering Conference, ITOEC 2017, pp. 790-793, October 2017.

[238] R. Stepanov, A. Dumler, S. Podtaev, and P. Frick, "Wavelet analysis in impedance rheocardiography," in Bioimpedance in Biomedical Applications and Research, pp. 257-269, Springer International Publishing, Cham, Switzerland, 2018.

[239] S. M. M. Naidu, U. R. Bagal, P. C. Pandey, S. Hardas, and N. D. Khambete, "Monitoring of stroke volume through impedance cardiography using an artificial neural network," in Proceedings of the 2015 21st National Conference on Communications, NCC 2015, pp. 1-6, March 2015.

[240] J. Muñoz, P. Gambús, E. W. Jensen, and M. Vallverdú, “Timefrequency features for impedance cardiography signals during anesthesia using different distribution kernels," Methods of Information in Medicine, vol. 57, no. S01, pp. el-e9, 2018.

[241] J. Escrivá Muñoz, Y. Pan, S. Ge, E. W. Jensen, and M. Vallverdú, "Novel characterization method of impedance cardiography signals using time-frequency distributions," Medical \& Biological Engineering \& Computing, vol. 56, no. 10, pp. 1757-1770, 2018.

[242] R. Beck, L. Milella, and C. Labellarte, "Continuous non-invasive measurement of stroke volume and cardiac index in infants and children: comparison of Impedance Cardiography NICaSOR vs CardioQOR method," La Clinica Terapeutica, vol. 169, no. 3, pp. el10-el13, 2018.

[243] P. Bogui, E. Balayssac-Siransy, P. Connes et al., "The physioflow thoracic impedancemeter is not valid for the measurements of cardiac hemodynamic parameters in chronic anemic patients," PLoS ONE, vol. 8, no. 10, 2013.

[244] M. Borzage, K. Heidari, T. Chavez et al., "Measuring stroke volume: Impedance cardiography vs phasecontrast magnetic resonance imaging," American Journal of Critical Care, vol. 26, no. 5, pp. 408-415, 2017.

[245] M. J. Germain, J. Joubert, D. O’Grady, B. H. Nathanson, Y. Chait, and N. W. Levin, "Comparison of stroke volume measurements during hemodialysis using bioimpedance cardiography and echocardiography," Hemodialysis International, vol. 22, no. 2, pp. 201-208, 2018.

[246] D. P. Bernstein, I. C. Henry, H. J. Lemmens et al., "Validation of stroke volume and cardiac output by electrical interrogation of the brachial artery in normals: assessment of strengths, limitations, and sources of error," Journal of Clinical Monitoring and Computing, vol. 29, no. 6, pp. 789-800, 2015.

[247] H. Liang, Å. Vårtun, and G. Acharya, "Agreement between preload reserve measured by impedance cardiography and echocardiography during pregnancy," Archives of Gynecology and Obstetrics, vol. 298, no. 1, pp. 59-66, 2018.

[248] J. M. van De Water, B. E. Mount, J. R. Barela, R. Schuster, and F. S. Leacock, "Monitoring the chest with impedance," Chest, vol. 64, no. 5, pp. 597-603, 1973.

[249] A. F. Pacela, "Impedance pneumography-A survey of instrumentation techniques," Medical \& Biological Engineering, vol. 4, no. 1, pp. 1-15, 1966.

[250] V. Jeyhani, T. Vuorinen, M. Mäntysalo, and A. Vehkaoja, “Comparison of simple algorithms for estimating respiration rate from electrical impedance pneumography signals in wearable devices," Health and Technology, vol. 7, no. 1, pp. 21-31, 2017.

[251] L. D’Alvia, E. Scalona, E. Palermo et al., "Tetrapolar lowcost systems for thoracic impedance plethysmography," in Proceedings of the 13th IEEE International Symposium on Medical Measurements and Applications, MeMeA 2018, pp. 1-6, June 2018.

[252] M. Młyńczak and G. Cybulski, "Impedance pneumography: Is it possible?" in Photonics Applications in Astronomy, Communications, Industry, and High-Energy Physics Experiments, vol. 8454, p. 84, 2012.

[253] M. H. Sim, M. Y. Kim, I. C. Jeong et al., "Development and evaluation of an improved technique for pulmonary function testing using electrical impedance pneumography intended for the diagnosis of chronic obstructive pulmonary disease patients," Sensors, vol. 13, no. 11, pp. 15846-15860, 2013.

[254] J. N. Wilkinson and V. U. Thanawala, "Thoracic impedance monitoring of respiratory rate during sedation - is it safe?" Anaesthesia, vol. 64, no. 4, pp. 455-456, 2009.

[255] V.-P. Seppä and J. Viik, Measuring Lung Volume Changes by Impedance Pneumography, Tampereen Teknillinen Yliopisto, 2015, Patent US 2015-0051469 A1.

[256] M. Młyńczak, W. Niewiadomski, M. Żyliński, and G. Cybulski, "Verification of the respiratory parameters derived from impedance pneumography during normal and deep breathing in three body postures," in 6th European Conference of the International Federation for Medical and Biological Engineering, I. Lacković and D. Vasic, Eds., vol. 45, pp. 881-884, Springer International Publishing, Cham, Switzerland, 2015.

[257] V. Seppä, A. S. Pelkonen, A. Kotaniemi-Syrjänen, J. Viik, M. J. Mäkelä, and L. P. Malmberg, "Tidal flow variability measured by impedance pneumography relates to childhood asthma risk," European Respiratory Journal, vol. 47, no. 6, pp. 1687-1696, 2016.

[258] T. Das, T. K. Dawn, P. Das, and P. Basak, "Design of low cost apnea detector by modifying a simple pulse delay circuit interfacing with computer with audio input by freely available 
audio software," in Proceedings of the 1st International Conference on Electronics, Materials Engineering and Nano-Technology, IEMENTech 2017, pp. 1-4, April 2017.

[259] K. Lu, L. Yang, F. Seoane, F. Abtahi, M. Forsman, and K. Lindecrantz, "Fusion of heart rate, respiration and motion measurements from a wearable sensor system to enhance energy expenditure estimation," Sensors, vol. 18, no. 9, 2018.

[260] Młyńczak M. and G. Cybulski, "Improvement of body posture changes detection during ambulatory respiratory measurements using impedance pneumography signals," in Proceedings of the 14 Mediterranean Conference on Medical and Biological Engineering and Computing, E. Kyriacou, S. Christofides, and C. S. Pattichis, Eds., pp. 167-171, Springer International Publishing, Cham, Switzerland, 2016.

[261] T. Koivumäki, J. Teuho, M. Teräs, M. Vauhkonen, and M. A. Hakulinen, "A novel respiratory gating method for oncologic positron emission tomography based on bioimpedance approach," Annals of Nuclear Medicine, vol. 29, no. 4, pp. 351358, 2015.

[262] T. Koivumäki, M. Vauhkonen, J. T. Kuikka, and M. A. Hakulinen, "Optimizing bioimpedance measurement configuration for dual-gated nuclear medicine imaging: A sensitivity study," Medical \& Biological Engineering \& Computing, vol. 49, no. 7, pp. 783-791, 2011.

[263] M. J. Kortelainen, T. M. Koivumäki, M. J. Vauhkonen et al., "Respiratory motion reduction with a dual gating approach in myocardial perfusion SPECT: Effect on left ventricular functional parameters," Journal of Nuclear Cardiology, vol. 25, no. 5, pp. 1633-1641, 2018.

[264] K. Tuohimäki, S. Mahdiani, V. Jeyhani et al., "Electrode comparison for textile-integrated electrocardiogram and impedance pneumography measurement," in Proceedings of the European Medical and Biological Engineering Confernce, Nordic-Baltic Conference on Biomedical Engineering and Medical Physics, $\mathrm{H}$. Eskola, O. Väisänen, J. Viik, and J. Hyttinen, Eds., pp. 302-305, Springer Singapore, Singapore, June 2018.

[265] P. Guay, S. Gorgutsa, S. Larochelle, and Y. Messaddeq, "Wearable contactless respiration sensor based on multi-material fibers integrated into textile," Sensors, vol. 17, no. 5, 2017.

[266] H. Li, H. Xu, Y. Ma et al., "Thoracic impedance measurement for lung function evaluation," in Proceedings of the 2017 IEEE International Conference on Imaging Systems and Techniques, IST 2017, pp. 1-5, China, October 2017.

[267] M. Młyńczak, M. Zyliński, D. Janczak, M. Jakubowska, W. Niewiadomski, and G. Cybulski, "Graphene electrodes for long-term impedance pneumography - A feasibility study," in Proceedings of the European Medical and Biological Engineering Confernce, Nordic-Baltic Conference on Biomedical Engineering and Medical Physics, H. Eskola, O. Väisänen, J. Viik, and J. Hyttinen, Eds., pp. 514-517, Springer Singapore, Singapore, 2018.

[268] S. Genc, Respiration Rate Determination in Impedance Pneumography, General Electric Company, 2017, Patent US 9-706945 B2.

[269] M. Młyńczak and G. Cybulski, "Decomposition of the cardiac and respiratory components from impedance pneumography signals," in Proceedings of the 10th International Joint Conference on Biomedical Engineering Systems and Technologies, vol. 4, pp. 16-23, Porto, Portugal, Feburary 2017.

[270] M. Młyńczak and G. Cybulski, "Flow parameters derived from impedance pneumography after nonlinear calibration based on neural networks," in Proceedings of the 10th International Joint
Conference on Biomedical Engineering Systems and Technologies, vol. 4, pp. 70-77, Porto, Portugal, Feburary 2017.

[271] K. van Loon, L. M. Peelen, E. C. van de Vlasakker, C. J. Kalkman, L. van Wolfswinkel, and B. van Zaane, "Accuracy of remote continuous respiratory rate monitoring technologies intended for low care clinical settings: a prospective observational study," Canadian Journal of Anesthesia, vol. 65, no. 12, pp. 1324-1332, 2018.

[272] B. G. Goudra, L. C. Penugonda, R. M. Speck, and A. C. Sinha, "Comparison of acoustic respiration rate, impedance pneumography and capnometry monitors for respiration rate accuracy and apnea detection during gi endoscopy anesthesia," Open Journal of Anesthesiology, vol. 3, no. 2, pp. 74-79, 2013.

[273] D. C. Barber and B. H. Brown, "Applied potential tomography," Journal of Physics E: Scientific Instruments, vol. 17, no. 9, pp. 723733, 1984.

[274] N. Li, H. Xu, Z. Zhou, J. Xin, Z. Sun, and X. Xu, "Reconfigurable bioimpedance emulation system for electrical impedance tomography system validation," IEEE Transactions on Biomedical Circuits and Systems, vol. 7, no. 4, pp. 460-468, 2013.

[275] T. Mauri, A. Mercat, and G. Grasselli, "What's new in electrical impedance tomography," Intensive Care Medicine, 2018.

[276] S. Zhang, G. Xu, X. Zhang et al., "Computation of a 3-D model for lung imaging with electrical impedance tomography," IEEE Transactions on Magnetics, vol. 48, no. 2, pp. 651-654, 2012.

[277] T. I. Oh, E. J. Woo, and D. Holder, "Multi-frequency EIT system with radially symmetric architecture: KHU Mark1," Physiological Measurement, vol. 28, no. 7, pp. S183-S196, 2007.

[278] R. Halter, A. Hartov, and K. Paulsen, "A broadband highfrequency electrical impedance tomography system for breast imaging," IEEE Transactions on Biomedical Engineering, vol. 55, no. 2, pp. 650-659, 2008.

[279] C. W. Denyer, F. J. Lidgey, Q. S. Zhu, and C. N. McLeod, "A high output impedance current source," Physiological Measurement, vol. 15, no. 2A, pp. A79-A82, 1994.

[280] X. Zhang, G. Xu, S. Zhang et al., "A numerical computation forward problem model of electrical impedance tomography based on generalized finite element method," IEEE Transactions on Magnetics, vol. 50, no. 2, pp. 1045-1048, 2014.

[281] X. Zhang, J. Liu, and B. He, "Magnetic-resonance-based electrical properties tomography: a review," IEEE Reviews in Biomedical Engineering, vol. 7, pp. 87-96, 2014.

[282] G. Singh, S. Anand, B. Lall, A. Srivastava, and V. Singh, "Development of a microcontroller based electrical impedance tomography system," in Proceedings of the 2015 IEEE Long Island Systems, Applications and Technology Conference, LISAT 2015, 2015.

[283] I. Frerichs, M. Amato, A. H. van Kaam et al., "Chest electrical impedance tomography examination, data analysis, terminology, clinical use and recommendations: consensus statement of the translational EIT development study group," Thorax, vol. 72, no. 1, pp. 83-93, 2017.

[284] J. C. Richard, C. Pouzot, A. Gros et al., "Electrical impedance tomography compared to positron emission tomography for the measurement of regional lung ventilation: An experimental study," Critical Care, vol. 13, no. 3, 2009.

[285] T. Dowrick, C. Blochet, and D. Holder, "In vivo bioimpedance measurement of healthy and ischaemic rat brain: Implications for stroke imaging using electrical impedance tomography," Physiological Measurement, vol. 36, no. 6, article no. 1273, pp. 1273-1282, 2015. 
[286] J. Zhang, B. Yang, H. Li et al., "A novel 3D-printed head phantom with anatomically realistic geometry and continuously varying skull resistivity distribution for electrical impedance tomography," Scientific Reports, vol. 7, no. 1, article no. 4608, 2017.

[287] F. Fu, B. Li, M. Dai et al., "Use of electrical impedance tomography to monitor regional cerebral edema during clinical dehydration treatment," PLoS ONE, vol. 9, no. 12, Article ID el13202, 2014.

[288] Y. Li, D. Zhang, B. Liu et al., "Noninvasive cerebral imaging and monitoring using electrical impedance tomography during total aortic arch replacement," Journal of Cardiothoracic and Vascular Anesthesia, vol. 32, no. 6, pp. 2469-2476, 2018.

[289] Y. Zhang, R. Xiao, and C. Harrison, "Advancing hand gesture recognition with high resolution electrical impedance tomography," in Proceedings of the 29th Annual Symposium on User Interface Software and Technology (UIST '16), pp. 843-850, ACM, New York, NY, USA, October 2016.

[290] S. Hong, K. Lee, U. Ha et al., "A $4.9 \mathrm{~m} \Omega$-sensitivity mobile electrical impedance tomography IC for early breast-cancer detection system," IEEE Journal of Solid-State Circuits, vol. 50, no. 1, pp. 245-257, 2015.

[291] E. K. Murphy, A. Mahara, and R. J. Halter, "Absolute reconstructions using rotational electrical impedance tomography for breast cancer imaging," IEEE Transactions on Medical Imaging, vol. 36, no. 4, pp. 892-903, 2017.

[292] K. Ain, R. A. Wibowo, and S. Soelistiono, "Modeling of electrical impedance tomography to detect breast cancer by finite volume methods," Journal of Physics: Conference Series, vol. 853, no. 1, Article ID 012001, 2017.

[293] P. Davies, S. Gates, D. Bird, and C. Silvestre, "Impedance tomography in paediatric intensive care patients," Pediatric Critical Care Medicine, vol. 19, no. 6S, p. 46, 2018.

[294] Y. Wu, D. Jiang, A. Bardill, S. de Gelidi, R. Bayford, and A. Demosthenous, "A high frame rate wearable EIT system using active electrode ASICs for lung respiration and heart rate monitoring," IEEE Transactions on Circuits and Systems I: Regular Papers, vol. 65, no. 11, pp. 3810-3820, 2018.

[295] M. Ma, Y. Zhang, and N. Gu, "Estimation the tumor temperature in magnetic nanoparticle hyperthermia by infrared thermography: Phantom and numerical studies," Journal of Thermal Biology, vol. 76, pp. 89-94, 2018.

[296] A. Mahara, S. Khan, E. K. Murphy, A. R. Schned, E. S. Hyams, and R. J. Halter, "3D microendoscopic electrical impedance tomography for margin assessment during robotassisted laparoscopic prostatectomy," IEEE Transactions on Medical Imaging, vol. 34, no. 7, pp. 1590-1601, 2015.

[297] T. K. Bera, "Applications of electrical impedance tomography (EIT): a short review," IOP Conference Series: Materials Science and Engineering, vol. 331, no. 1, Article ID 012004, 2018.

[298] K. Zhang, M. Li, F. Yang, S. Xu, and A. Abubakar, "Study on the accuracy of forward modeling in electrical impedance tomography for thorax imaging," in Proceedings of the 32nd General Assembly and Scientific Symposium of the International Union of Radio Science, URSI GASS 2017, pp. 1-4, August 2017.

[299] E. Ma, "Convergence of finite element approximation for electrical impedance tomography with the complete electrode model," Journal of Physics Communications, vol. 2, no. 8, Article ID 085024, 2018.

[300] N. Hyvönen and L. Mustonen, "Generalized linearization techniques in electrical impedance tomography," Numerische Mathematik, vol. 140, no. 1, pp. 95-120, 2018.
[301] K. Knudsen, M. Lassas, J. Mueller, and S. Siltanen, "Regularized D-bar method for the inverse conductivity problem," Inverse Problems and Imaging, vol. 35, no. 4, pp. 599-624, 2009.

[302] A. P. Calderón, "On an inverse boundary value problem," Computational \& Applied Mathematics, vol. 25, no. 2-3, pp. $133-$ 138, 2006.

[303] S. J. Hamilton and A. Hauptmann, "Deep D-bar: real-time electrical impedance tomography imaging with deep neural networks," IEEE Transactions on Medical Imaging, vol. 37, no. 10, pp. 2367-2377, 2018.

[304] X. Li, Y. Lu, J. Wang et al., "An image reconstruction framework based on deep neural network for electrical impedance tomography," in Proceedings of the 24th IEEE International Conference on Image Processing, ICIP 2017, pp. 3585-3589, September 2017.

[305] G. Kłosowski and T. T. Rymarczyk, "Using neural networks and deep learning algorithms in electrical impedance tomography," Informatyka, Automatyka, Pomiary $w$ Gospodarce i Ochronie Środowiska, 2017.

[306] A. Samore, M. Guermandi, S. Placati, and R. Guerrieri, "Parametric detection and classification of compact conductivity contrasts with electrical impedance tomography," IEEE Transactions on Instrumentation and Measurement, vol. 66, no. 10, pp. 2666-2679, 2017.

[307] L. Zhou, B. Harrach, and J. K. Seo, "Monotonicity-based electrical impedance tomography for lung imaging," Inverse Problems, vol. 34, no. 4, Article ID 045005, 2018.

[308] B. Harrach and M. N. Minh, "Monotonicity-based regularization for phantom experiment data in electrical impedance tomography," in New Trends in Parameter Identification for Mathematical Models, pp. 107-120, Springer International Publishing, 2018.

[309] H. Garde and S. Staboulis, "Convergence and regularization for monotonicity-based shape reconstruction in electrical impedance tomography," Numerische Mathematik, vol. 135, no. 4, pp. 1221-1251, 2017.

[310] T. Rymarczyk and K. Szulc, "Solving inverse problem for electrical impedance tomography using topological derivative and level set method," in Proceedings of the 2018 International Interdisciplinary PhD Workshop, IIPhDW 2018, pp. 191-195, May 2018.

[311] S. P. Pellegrini, F. C. Trigo, and R. G. Lima, "Solving the electrical impedance tomography inverse problem for logarithmic conductivity: Numerical sensitivity," COMPEL - The International Journal for Computation and Mathematics in Electrical and Electronic Engineering, vol. 37, no. 2, pp. 681-690, 2018.

[312] R. A. Borsoi, J. C. C. Aya, G. H. Costa, and J. C. M. Bermudez, "Super-resolution reconstruction of electrical impedance tomography images," Computers and Electrical Engineering, vol. 69, pp. 1-13, 2018.

[313] S. Liu, J. Jia, Y. D. Zhang, and Y. Yang, "Image reconstruction in electrical impedance tomography based on structure-aware sparse bayesian learning," IEEE Transactions on Medical Imaging, vol. 37, no. 9, pp. 2090-2102, 2018.

[314] M. Kim, J. Jang, H. Kim et al., “A 1.4-m $\Omega$-Sensitivity 94-dB dynamic-range electrical impedance tomography SoC and 48channel Hub-SoC for 3-D lung ventilation monitoring system," IEEE Journal of Solid-State Circuits, vol. 52, no. 11, pp. 28292842, 2017.

[315] M. Rapin, F. Braun, A. Adler et al., "Wearable sensors for frequency-multiplexed EIT and multilead ECG data acquisition," IEEE Transactions on Biomedical Engineering, 2018. 
[316] Y. Yang, J. Jia, S. Smith, N. Jamil, W. Gamal, and P. Bagnaninchi, "A miniature electrical impedance tomography sensor and 3-D image reconstruction for cell imaging," IEEE Sensors Journal, vol. 17, no. 2, pp. 514-523, 2017.

[317] Y. Yang and J. Jia, "A multi-frequency electrical impedance tomography system for real-time 2D and 3D imaging," Review of Scientific Instruments, vol. 88, no. 8, Article ID 085110, 2017.

[318] A. Dupré and S. Mylvaganam, "A simultaneous and continuous excitation method for high-speed electrical impedance tomography with reduced transients and noise sensitivity," Sensors, vol. 18 , no. $4,2018$.

[319] J. Avery, T. Dowrick, M. Faulkner, N. Goren, and D. Holder, "A versatile and reproducible multi-frequency electrical impedance tomography system," Sensors, vol. 17, no. 2, 2017.

[320] H. D. Critchley, R. Elliott, C. J. Mathias, and R. J. Dolan, "Neural activity relating to generation and representation of galvanic skin conductance responses: a functional magnetic resonance imaging study," The Journal of Neuroscience, vol. 20, no. 8, pp. 3033-3040, 2000.

[321] R. L. Bailey, "Electrodermal activity (EDA)," in The International Encyclopedia of Communication Research Methods, American Cancer Society, pp. 1-15, 2017.

[322] M. Velana, S. Gruss, G. Layher et al., "The senseemotion database: a multimodal database for the development and systematic validation of an automatic pain- and emotionrecognition system," in Multimodal Pattern Recognition of Social Signals in Human-Computer-Interaction, F. Schwenker and S. Scherer, Eds., pp. 127-139, Springer International Publishing, Cham, Switzerland, 2017.

[323] H. Critchley and Y. Nagai, "Electrodermal activity (EDA)," in Encyclopedia of Behavioral Medicine, pp. 666-669, Springer, 2013.

[324] S. Walter, S. Gruss, H. Ehleiter et al., "The biovid heat pain database data for the advancement and systematic validation of an automated pain recognition system," in Proceedings of the 2013 IEEE International Conference on Cybernetics (CYBCO), pp. 128-131, June 2013.

[325] J. Hernandez, R. R. Morris, and R. W. Picard, "Call center stress recognition with person-specific models," in Affective Computing and Intelligent Interaction, S. D’Mello, A. Graesser, B. Schuller, and J.-C. Martin, Eds., pp. 125-134, Springer, Berlin, Germany, 2011.

[326] R. Kocielnik, N. Sidorova, F. M. Maggi, M. Ouwerkerk, and J. H. D. M. Westerink, "Smart technologies for long-term stress monitoring at work," in Proceedings of the 26th IEEE International Symposium on Computer-Based Medical Systems, CBMS 2013, pp. 53-58, Portugal, June 2013.

[327] J. Shukla, M. Barreda-Ángeles, J. Oliver, and D. Puig, "Efficient wavelet-based artifact removal for electrodermal activity in real-world applications," Biomedical Signal Processing and Control, vol. 42, pp. 45-52, 2018.

[328] S. Taylor, N. Jaques, W. Chen, S. Fedor, A. Sano, and R. Picard, "Automatic identification of artifacts in electrodermal activity data," in Proceedings of the 37th Annual International Conference of the IEEE Engineering in Medicine and Biology Society, EMBC 2015, pp. 1934-1937, August 2015.

[329] M. Kelsey, M. Akcakaya, I. R. Kleckner et al., "Applications of sparse recovery and dictionary learning to enhance analysis of ambulatory electrodermal activity data," Biomedical Signal Processing and Control, vol. 40, pp. 58-70, 2018.
[330] H. F. Posada-Quintero, N. Reljin, C. Mills et al., "Time-varying analysis of electrodermal activity during exercise," PLoS ONE, vol. 13, no. 6, pp. 1-12, 2018.

[331] H. F. Posada-Quintero and K. H. Chon, "Exploring electrodermal activity in water-immersed subjects," in Proceedings of the 15th IEEE International Conference on Wearable and Implantable Body Sensor Networks, BSN 2018, pp. 90-93, March 2018.

[332] A. Greco, G. Valenza, L. Citi, and E. P. Scilingo, "Arousal and valence recognition of affective sounds based on electrodermal activity," IEEE Sensors Journal, vol. 17, no. 3, pp. 716-725, 2017.

[333] S. Jain, U. Oswal, K. S. Xu, B. Eriksson, and J. Haupt, "A compressed sensing based decomposition of electrodermal activity signals," IEEE Transactions on Biomedical Engineering, vol. 64, no. 9, pp. 2142-2151, 2017.

[334] S. G. Caliskan, M. D. Bilgin, and M. Polatli, "Nonlinear analysis of electrodermal activity signals for healthy subjects and patients with chronic obstructive pulmonary disease," Australasian Physical \& Engineering Sciences in Medicine, vol. 41, no. 2, pp. 487-494, 2018.

[335] R. Zangróniz, A. Martínez-Rodrigo, J. M. Pastor, M. T. López, and A. Fernández-Caballero, "Electrodermal activity sensor for classification of calm/distress condition," Sensors, vol. 17, no. 10, 2017.

[336] D. McDonnell, Electrodermal Activity Sensor, Galvanic Ltd., 2017, Patent US 2017-0014043 A1.

[337] S. Heiermann, K. Khalaj Hedayati, M. J. Müller, and M. Dittmar, "Accuracy of a portable multisensor body monitor for predicting resting energy expenditure in older people: a comparison with indirect calorimetry," Gerontology, vol. 57, no. 5, pp. 473-479, 2011.

[338] H. Mayaudon, P. Miloche, and B. Bauduceau, "A new simple method for assessing sudomotor function: Relevance in type 2 diabetes," Diabetes \& Metabolism, vol. 36, no. 6, pp. 450-454, 2010.

[339] C. Karagiannidis, A. D. Waldmann, P. L. Róka et al., "Regional expiratory time constants in severe respiratory failure estimated by electrical impedance tomography: A feasibility study," Critical Care, vol. 22, no. 1, 2018.

[340] J. Karsten, N. Voigt, H.-J. Gillmann, and T. Stueber, "Determination of optimal positive end-expiratory pressure based on respiratory compliance and electrical impedance tomography: A pilot clinical comparative trial," Biomedizinische Technik. Biomedical Engineering, 2018.

[341] C. Shi, S. Boehme, A. H. Bentley et al., "Assessment of regional ventilation distribution: comparison of vibration response imaging (VRI) with electrical impedance tomography (EIT)," PLoS ONE, vol. 9, no. 1, 2014.

[342] E. Barsoukov and J. Macdonald, Impedance Spectroscopy: Theory, Experiment, and Applications, Wiley-Interscience, Hoboken, NJ, USA, 3rd edition, 2018.

[343] S. Sang, Y. Wang, Q. Feng, Y. Wei, J. Ji, and W. Zhang, "Progress of new label-free techniques for biosensors: A review," Critical Reviews in Biotechnology, vol. 36, no. 3, pp. 465-481, 2016.

[344] M. Min, T. Parve, and U. Pliquett, "Impedance detection," in Encyclopedia of Microfluidics and Nanofluidics, pp. 1338-1361, Springer, New York, NY, USA, 2nd edition, 2015.

[345] A. De Ninno, V. Errico, F. R. Bertani, L. Businaro, P. Bisegna, and F. Caselli, "Coplanar electrode microfluidic chip enabling accurate sheathless impedance cytometry," Lab on a Chip, vol. 17, no. 6, pp. 1158-1166, 2017. 
[346] M. Varshney and Y. Li, "Interdigitated array microelectrodes based impedance biosensors for detection of bacterial cells," Biosensors and Bioelectronics, vol. 24, no. 10, pp. 2951-2960, 2009.

[347] C. Vericat, M. E. Vela, G. Benitez, P. Carro, and R. C. Salvarezza, "Self-assembled monolayers of thiols and dithiols on gold: new challenges for a well-known system," Chemical Society Reviews, vol. 39, no. 5, pp. 1805-1834, 2010.

[348] M. Min, R. Land, T. Paavle, T. Parve, P. Annus, and D. Trebbels, "Broadband spectroscopy of dynamic impedances with short chirp pulses," Physiological Measurement, vol. 32, no. 7, pp. 945958, 2011.

[349] M. Chua, "Design and development of an integrated health (ihealth) monitoring watch," in Proceedings of the 2016 IEEE/SICE International Symposium on System Integration, pp. 296-300, 2017.

[350] M. Metshein and R. Gordon, "On the possibility of detecting the electrical bioimpedance of human body by using non-contact electrodes in capacitive connection," in Proceedings of the 15th Biennial Baltic Electronics Conference, BEC 2016, vol. 2016, pp. 171-174, October 2016.

[351] S. Rossi, C. Mancarella, C. Mocenni, and L. Della Torre, "Bioimpedance sensing in wearable systems: From hardware integration to model development," in Proceedings of the $3 \mathrm{rd}$ IEEE International Forum on Research and Technologies for Society and Industry, RTSI 2017, September 2017.

[352] P. De Carvalho, J. Palacio, and W. Van Noije, Area Optimized CORDIC-Based Numerically Controlled Oscillator for Electrical Bioimpedance Spectroscopy, 2016.

[353] I. Kim, Y. A. Bhagat, J. Homer, and R. Lobo, "Multimodal analog front end for wearable bio-sensors," IEEE Sensors Journal, vol. 16, no. 24, pp. 8784-8791, 2016.

[354] I. Kukharenko and V. Kotovskyi, "Low power bioimpedance tracking system for stress and activity monitoring," in Proceedings of the 37th IEEE International Conference on Electronics and Nanotechnology, ELNANO 2017, pp. 288-291, April 2017.

[355] D. Allegri, D. Vaca, D. Ferreira, M. Rogantini, and D. Barrettino, "Real-time monitoring of the hydration level by multifrequency bioimpedance spectroscopy," in Proceedings of the 2017 IEEE International Instrumentation and Measurement Technology Conference, I2MTC 2017, May 2017.

[356] R. Kumar, S. Perumalla, J. Vista, and A. Ranjan, "Realization of single and double cole tissue models using higher order approximation," in Proceedings of the 1st International Conference on Electronics, Materials Engineering and Nano-Technology, IEMENTech 2017, April 2017.

[357] P. Kassanos and G.-Z. Yang, "A CMOS programmable phase shifter for compensating synchronous detection bioimpedance systems," in Proceedings of the 24th IEEE International Conference on Electronics, Circuits and Systems, ICECS 2017, vol. 2018, pp. 218-221, 2018.

[358] D. Naranjo, L. M. Roa, L. J. Reina et al., "Analysis of anomalies in bioimpedance models for the estimation of body composition," in Proceedings of the 13th Mediterranean Conference on Medical and Biological Engineering and Computing 2013 IFMBE, vol. 41, pp. 1563-1566, 2013.

[359] C. A. Shiffman, "Adverse effects of near current-electrode placement in non-invasive bio-impedance measurements," Physiological Measurement, vol. 34, no. 11, pp. 1513-1529, 2013.

[360] H. Kwon, J. A. Nagy, R. Taylor, S. B. Rutkove, and B. Sanchez, "New electrical impedance methods for the in situ measurement of the complex permittivity of anisotropic biological tissues," Physics in Medicine and Biology, vol. 62, no. 22, pp. 8616-8633, 2017.

[361] Z. Wu, Y. Liu, M. Hong, and X. Yu, "A review of anisotropic conductivity models of brain white matter based on diffusion tensor imaging," Medical \& Biological Engineering \& Computing, vol. 56, no. 8, pp. 1325-1332, 2018.

[362] K. Badstuebner, M. Stubbe, T. Kroeger, E. Mix, and J. Gimsa, "Impedance detection of the electrical resistivity of the wound tissue around deep brain stimulation electrodes permits registration of the encapsulation process in a rat model," Journal of Electrical Bioimpedance (JEB), vol. 8, no. 1, pp. 11-24, 2017.

[363] J. Anso, T. Wyss-Balmer, Y. Jegge et al., "Electrical impedance to assess facial nerve proximity during robotic cochlear implantation," IEEE Transactions on Biomedical Engineering, 2018.

[364] C.-H. Chuang and M. Shaikh, "Label-free impedance biosensors for Point-of-Care diagnostics," in Point-of-Care Diagnostics - New Progresses and Perspectives, pp. 171-201, IAPC Publishing, Zagreb, Croatia, 2017.

[365] P. Kassanos, S. Anastasova, and G.-Z. Yang, "Electrical and physical sensors for biomedical implants," in Implantable Sensors and Systems: From Theory to Practice, pp. 99-195, 2018.

[366] A. S. Anusha, S. P. Preejith, J. Joseph, and M. Sivaprakasam, "Design and preliminary analysis of a multifrequency bioimpedance measurement scheme," in Proceedings of the 11th IEEE International Symposium on Medical Measurements and Applications, MeMeA 2016, May 2016.

[367] A. Montalibet, W. Arkouche, P. B. Franco et al., "Localised impedance monitoring device for the remote clinical assessment of home-based dialysis patients," in Proceedings of the 38th Annual International Conference of the IEEE Engineering in Medicine and Biology Society, EMBC 2016, pp. 4427-4430, USA, August 2016.

[368] D. J. Wang and S. S. Gottlieb, "Impedance cardiography: More questions than answers," Current Cardiology Reports, vol. 8, no. 3, pp. 180-186, 2006.

[369] D. Buxi, E. Hermeling, M. Mercuri et al., "Systolic time interval estimation using continuous wave radar with on-body antennas," IEEE Journal of Biomedical and Health Informatics, vol. 22, no. 1, pp. 129-139, 2018.

[370] A. Ayadi, R. Ben Salah, K. Ouni, and W. Sahtout, "Determination of cardiovascular parameters from bioimpedance signal," in Proceedings of the 2nd International Conference on Advanced Technologies for Signal and Image Processing, ATSIP 2016, pp. 403-407, March 2016.

[371] R. Z. Rusho and M. A. Kadir, "Reconstruction algorithm for Pigeon Hole Imaging (PHI)," in Proceedings of the 3rd International Conference on Electrical Information and Communication Technology, EICT 2017, vol. 2018, pp. 1-6, 2018.

[372] L. Miao, Y. Ma, X. Chen et al., "A new symmetric semiparallel electrical impedance tomography (EIT) system - II: The performance," in Proceedings of the 2016 IEEE International Conference on Imaging Systems and Techniques, IST 2016, pp. 283-288, October 2016.

[373] N. Jamil, Y. Yang, A. Tsiamis, J. Jia, and S. Smith, "Comparison of regularisation methods in image reconstruction for microbioimpedance tomography," in Proceedings of the 16th IEEE SENSORS Conference, ICSENS 2017, vol. 2017, pp. 1-3, UK, November 2017.

[374] V. G. Sirtoli, K. Morcelles, and P. Bertemes-Filho, "Electrical properties of phantoms for mimicking breast tissue," in Proceedings of the 39th Annual International Conference of the IEEE 
Engineering in Medicine and Biology Society, EMBC 2017, pp. 157-160, July 2017.

[375] J. Jang, M. Kim, J. Bae, and H. Yoo, “A 2.79-mW 0.5\%-THD CMOS current driver IC for portable electrical impedance tomography system," in Proceedings of the 2017 IEEE Asian Solid-State Circuits Conference (A-SSCC), vol. 2017, pp. 145-148, November 2017.

[376] Z. Xu, J. Yao, Z. Wang et al., "Development of a portable electrical impedance tomography system for biomedical applications," IEEE Sensors Journal, vol. 18, no. 19, pp. 8117-8124, 2018.

[377] S. Leonhardt, L. Leicht, and D. Teichmann, "Unobtrusive vital sign monitoring in automotive environments-A review," Sensors, vol. 18, no. 9, 2018.

[378] T. H. Huynh, R. Jafari, and W.-Y. Chung, "A robust bioimpedance structure for smartwatch-based blood pressure monitoring," Sensors, vol. 18, no. 7, 2018.

[379] D. Buxi, J. Redout, and M. R. Yuce, "Blood pressure estimation using pulse transit time from bioimpedance and continuous wave radar," IEEE Transactions on Biomedical Engineering, vol. 64, no. 4, pp. 917-927, 2017.

[380] B. Ibrahim, V. Nathan, and R. Jafari, "Exploration and validation of alternate sensing methods for wearable continuous pulse transit time measurement using optical and bioimpedance modalities," in Proceedings of the 39th Annual International Conference of the IEEE Engineering in Medicine and Biology Society, EMBC 2017, pp. 2051-2055, July 2017.

[381] M. Min, P. Annus, H. Koiv, A. Krivosei, T. Uuetoa, and J. Lamp, "Bioimpedance sensing - A viable alternative for tonometry in non-invasive assessment of central aortic pressure," in Proceedings of the 12th IEEE International Symposium on Medical Measurements and Applications, MeMeA 2017, pp. 373-378, May 2017.

[382] B. Sanchez and S. B. Rutkove, "Electrical impedance myography and its applications in neuromuscular disorders," Neurotherapeutics, vol. 14, no. 1, pp. 107-118, 2017.

[383] J. Li, T. Igbe, Y. Liu et al., "An approach for noninvasive blood glucose monitoring based on bioimpedance difference considering blood volume pulsation," IEEE Access, vol. 6, pp. 51119-51129, 2018.

[384] T. Das, A. Ghosh, S. Guha, and P. Basak, "Early detection of diabetes based on skin impedance spectrogram and heart rate variability noninvasively," in Proceedings of the 1st International Conference on Electronics, Materials Engineering and NanoTechnology, IEMENTech 2017, April 2017.

[385] Q. Wang, H. Wang, H. Xu, W. Zhou, and G. Liu, "Noninvasive urination-desire sensing method based on bladder bioimpedance spectrum analysis," Journal of Medical and Biological Engineering, vol. 36, no. 2, pp. 191-196, 2016.

[386] E. Pittella, S. Pisa, E. Piuzzi, E. Rizzuto, and Z. Del Prete, "Combined impedance plethysmography and spectroscopy for the diagnosis of diseases of peripheral vascular system," in Proceedings of the 12th IEEE International Symposium on Medical Measurements and Applications, MeMeA 2017, pp. 367-372, May 2017.

[387] L. RenHe and B. Wongkittisuksa, "The evaluation of elbow joint motion using bio-impedance signal," in Proceedings of the 14th International Conference on Electrical Engineering/Electronics, Computer, Telecommunications and Information Technology, ECTI-CON 2017, pp. 262-265, June 2017.

[388] K. Kikkeri and M. Agah, "A monolithic dielectrophoretic chip for real-time low-abundance bacteria detection," in Proceedings of the 30th IEEE International Conference on Micro Electro Mechanical Systems, MEMS 2017, pp. 358-361, January 2017.

[389] A. Kekonen, M. Bergelin, J.-E. Eriksson, H. Ylanen, S. Kielosto, and J. Viik, "Bioimpedance measurement system for evaluation of the status of wound healing," in Proceedings of the 15th Biennial Baltic Electronics Conference, BEC 2016, vol. 2016, pp. 175-178, October 2016.

[390] J. A. Jambulingam, R. McCrory, L. West, and O. T. Inan, "Noninvasive, multi-modal sensing of skin stretch and bioimpedance for detecting infiltration during intravenous therapy," in Proceedings of the 38th Annual International Conference of the IEEE Engineering in Medicine and Biology Society, EMBC 2016, vol. 2016, pp. 4755-4758, August 2016.

[391] D. Celinskis and B. C. Towe, "Characterization of the implantable neurostimulator-based wireless bioimpedance measurement technique," in Proceedings of the 2016 IEEE EMBS International Student Conference, ISC 2016, May 2016.

[392] H. Kalvoy, C. Tronstad, K. Ullensvang, T. Steinfeldt, and A. R. Sauter, "Detection of needle to nerve contact based on electric bioimpedance and machine learning methods," in Proceedings of the 39th Annual International Conference of the IEEE Engineering in Medicine and Biology Society, EMBC 2017, pp. 9-12, July 2017. 


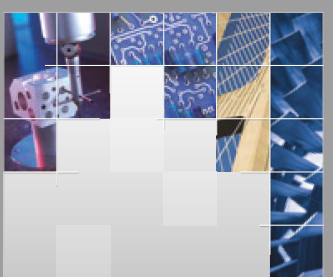

\section{Enfincering}
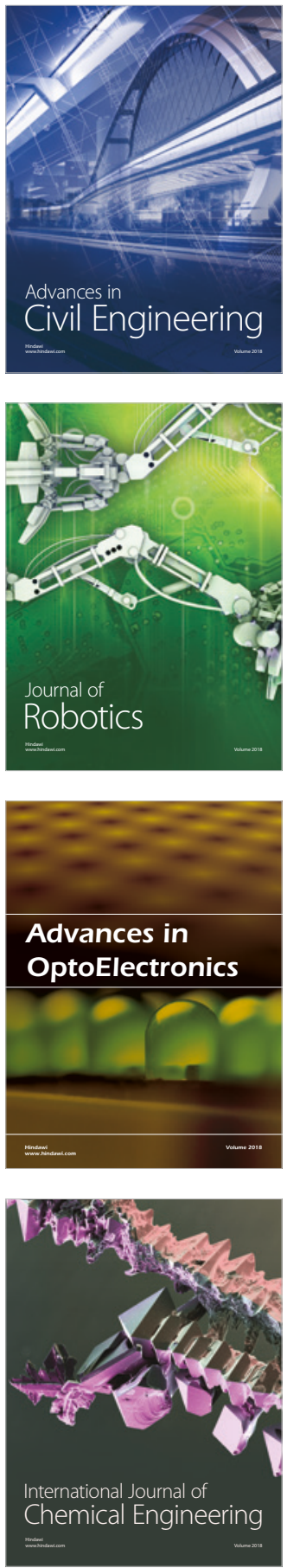

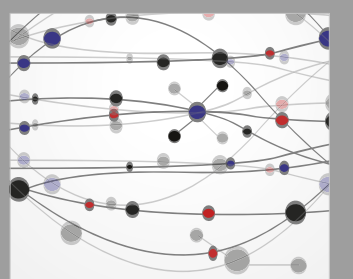

\section{Rotating \\ Machinery}

The Scientific World Journal

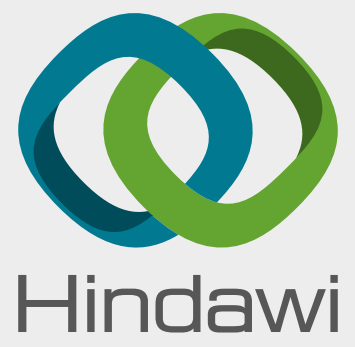

Submit your manuscripts at

www.hindawi.com
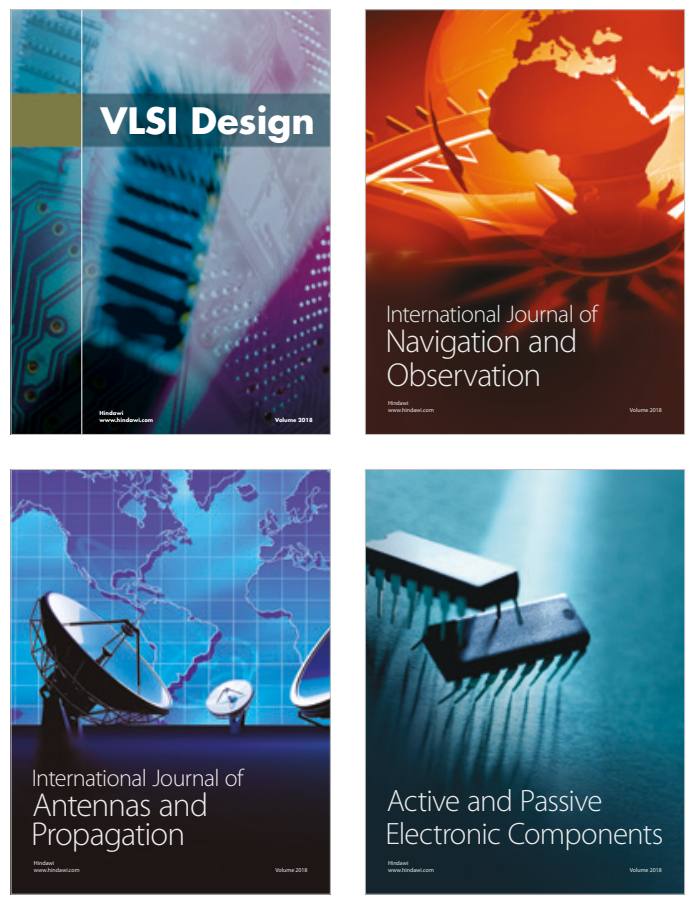
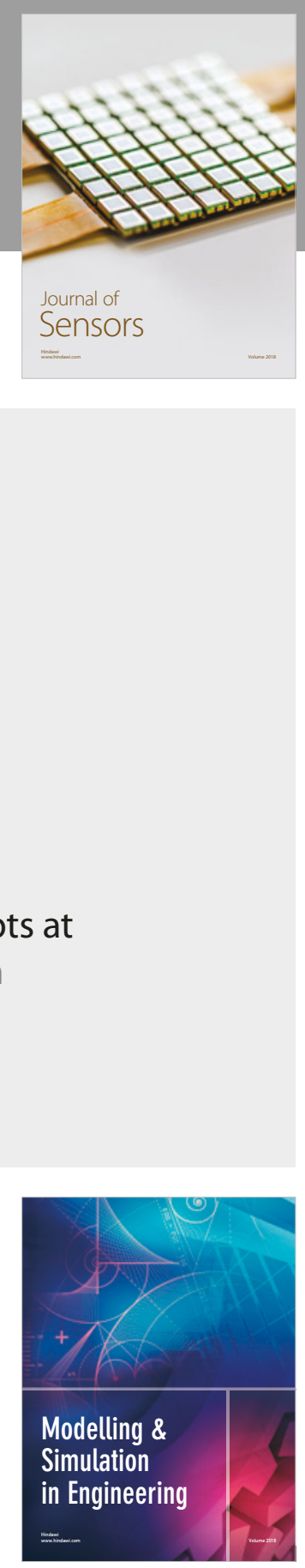

\section{Advances \\ Multimedia}
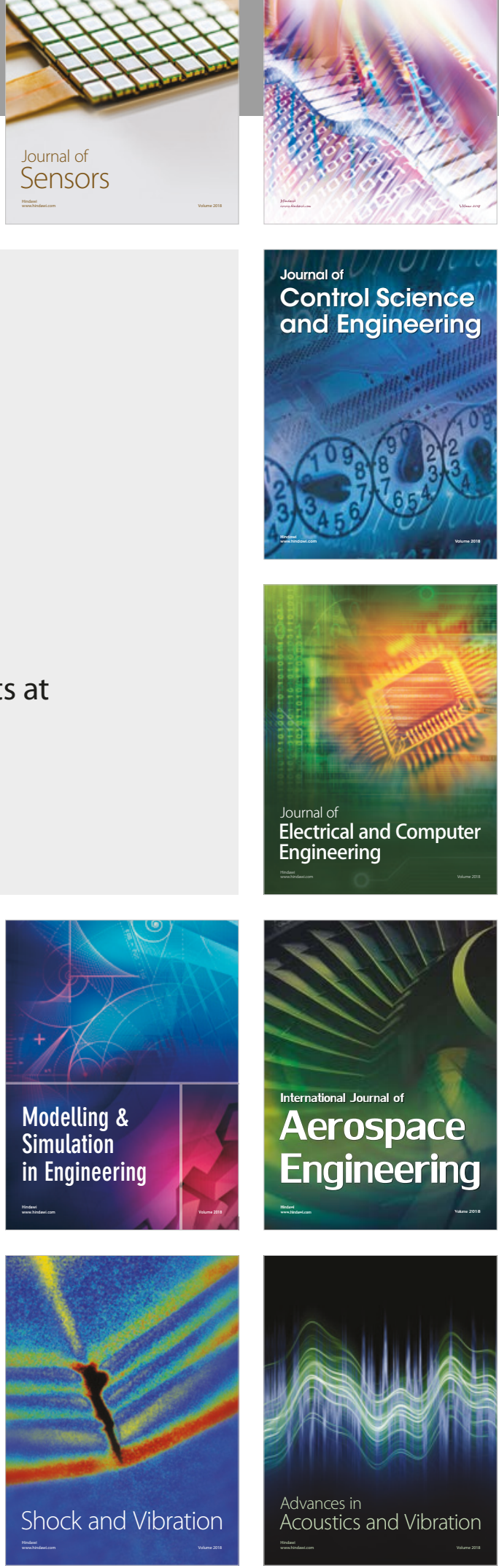